Supporting Information for:

\title{
Oxidative Kinetic Resolution of Acyclic Amines Based on Equilibrium Control
}

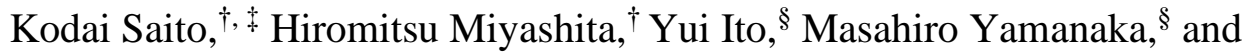 \\ Takahiko Akiyama*, ${ }^{\dagger}$
}

\footnotetext{
'Department of Chemistry, Faculty of Science, Gakushuin University, 1-5-1 Mejiro, Toshima-ku, Tokyo 171-8588, Japan

Department of Chemistry, Faculty of Science and Technology, Keio University, 3-14-1 Hiyoshi, Kohoku-ku, Yokohama, Kanagawa 223-8522, Japan

${ }^{\S}$ Department of Chemistry, Faculty of Science, Rikkyo University, 3-34-1 Nishi-Ikebukuro, Toshima-ku, Tokyo 171-8501, Japan
} 


\section{Table of Contents}

1. Syntheses and characterization data of starting materials

2. General procedure for the phosphoric acid catalyzed kinetic resolution and characterization data of acyclic amines

3. Computational details

4. Cartesian coordinates

5. ${ }^{1} \mathrm{H}$ NMR, ${ }^{13} \mathrm{C}$ NMR, and HPLC data of the reaction products and new compounds

6. References

General. NMR spectra were recorded on Unity Inova-400 or JNM-AL400 using $\mathrm{CDCl}_{3}$ as a solvent. Tetramethylsilane (TMS) $(\delta=0)$ or $\mathrm{CHCl}_{3}(\delta=7.26)$ served as an internal standard for ${ }^{1} \mathrm{H} \mathrm{NMR}$, and $\mathrm{CDCl}_{3}$ was used as an internal standard $(\delta=77.0)$ for ${ }^{13} \mathrm{C}$ NMR. Melting point (mp) determinations were performed by using a AS ONE ATM-01 instrument and are uncorrected. Infrared (IR) spectra were recorded on a FTIR-8600PC instrument (Shimadzu Co.). Electron spray ionization (ESI) mass spectra were recorded on a Shimadzu LCMS_2010 eV spectrometer or Bruker Daltonics microTOF_15 focus. EI mass spectra were recorded on JEOL GCmate ${ }^{\mathrm{TM}}$ II GC/MS Double-Focusing Mass Spectrometer. Optical rotations were measured on a HORIBA SEPA-500 polarimeter. Purification of the products was performed by column chromatography on silica gel (Fuji sylisia D60L or PSQ-60B) or preparative TLC on silica gel (Wako gel B-5F). All solvents were purified according to the standard procedures.

\section{Syntheses and characterization data of starting materials Syntheses of imines}

Imines $\mathbf{3} \mathbf{b}^{1}$ and $\mathbf{4} \mathbf{a}^{2}$ are known compounds.

Imine $\mathbf{3} \mathbf{b}^{1}$ was synthesized by the following procedure.

A magnetic stirrer bar and $5 \AA$ MS (ca. $4.0 \mathrm{~g}$ ) were placed in a two-necked round bottom flask $(100 \mathrm{~mL})$ under nitrogen atmosphere. The $5 \AA$ MS were then dried with a heat gun under reduced pressure and the flask was refilled with nitrogen. Benzaldehyde $(1.06 \mathrm{~g}$, $10.0 \mathrm{mmol})$, $p$-methoxyaniline $(1.23 \mathrm{~g}, 10.0 \mathrm{mmol})$, and dry benzene $(10 \mathrm{~mL})$ were added successively under nitrogen atmosphere at room temperature. After being stirred for 5 days at room temperature, the mixture was filtered through Celite (washed with $n$-hexane (ca. $100 \mathrm{~mL}$ )) and the precipitate was formed in the filtrate during the filtration. The precipitate was obtained by the filtration to give $1.81 \mathrm{~g}(8.57 \mathrm{mmol}, 86 \%)$ of $\mathbf{3 b}$ as light brown solid. 
Imines $\mathbf{3 a}$ and $\mathbf{4} \mathbf{a}^{2}$ were synthesized by the following procedure.

Benzaldehyde or acetophenone $(10.0 \mathrm{mmol})$ and 3,4,5-trimethoxyaniline $(10.0 \mathrm{mmol})$ was mixed in benzene $(7.0 \mathrm{~mL})$ in the presence of flame-dried $5 \AA$ MS (ca. $4.0 \mathrm{~g}$ ) under nitrogen atmosphere at $50{ }^{\circ} \mathrm{C}(\mathbf{3 a})$ and $80{ }^{\circ} \mathrm{C}(\mathbf{4 a})$ in oil bath for 5 days, respectively. After Celite filtration (washed with $\mathrm{CH}_{2} \mathrm{Cl}_{2}$ ), volatile materials were evaporated under reduced pressure and the crude material was purified by flash column chromatography on silica gel (eluent, AcOEt $/$ Hexane $=1 / 5$ with $1 \% \mathrm{NEt}_{3}$ ) and the obtained yellow solid was washed with hexane and dried in vacuo. 3a or $4 \mathbf{a}$ were obtained in $60 \%$ or $63 \%$ yields as light yellow solid, respectively. The synthesis and characterization data for $\mathbf{4 a}$ was previously reported. ${ }^{2}$<smiles>COc1cc(/N=C/c2ccccc2)cc(OC)c1OC</smiles>

3a $(60 \%)$

${ }^{1} \mathrm{H} \mathrm{NMR}\left(400 \mathrm{MHz}, \mathrm{CDCl}_{3}\right.$ ) $\delta: 3.86$ (s 3H), 3.89 (s $\left.6 \mathrm{H}\right), 6.49$ (s, 2H), 7.45-7.51 (m, 3H), 7.87-7.92 (m, 2H), $8.47(\mathrm{~s}, \mathrm{H})$.

${ }^{13} \mathrm{C}$ NMR $\left(100 \mathrm{MHz}, \mathrm{CDCl}_{3}\right)$ \&: 56.0, 60.9, 98.1, 128.69, 128.73, 131.4, 136.0, 16.3, $147.8,153.5,159.7$.

IR (film): $1585,1501,1231,1127 \mathrm{~cm}^{-1}$.

HRMS (ESI) $m / z$ calcd for $\mathrm{C}_{16} \mathrm{H}_{17} \mathrm{NO}_{3}(\mathrm{M})^{+} 271.1212$, found 271.1208.

m. p. $120-121{ }^{\circ} \mathrm{C}$.<smiles>COc1cc(/N=C(\C)c2ccccc2)cc(OC)c1OC</smiles>

$4 \mathbf{a}(63 \%)^{2}$

${ }^{1} \mathrm{H}$ NMR (400 MHz, $\left.\mathrm{CDCl}_{3}\right) \delta: 2.28(\mathrm{~s}, 3 \mathrm{H}), 3.83(\mathrm{~s}, 6 \mathrm{H}), 3.85(\mathrm{~s}, 3 \mathrm{H}), 6.03(\mathrm{~s}, 2 \mathrm{H})$, $7.40-7.48$ (m, $3 \mathrm{H}), 7.94-7.98$ (m, $2 \mathrm{H})$. 


\section{Syntheses of amines}

All acyclic amines were synthesized from corresponding aldehydes or ketones with anilines by the following indirect reductive amination.

Ketone $(5.0 \mathrm{mmol})$ and $p$-methoxyaniline $(5.0 \mathrm{mmol})$ was mixed in benzene $(5.0 \mathrm{~mL})$ in the presence of flame-dried $5 \AA$ MS (ca. $2.0 \mathrm{~g}$ ) under nitrogen atmosphere at room temperature for 3 days. After Celite filtration (washed with $\mathrm{CH}_{2} \mathrm{Cl}_{2}$ ), volatile materials were evaporated under reduced pressure and the crude material was treated with $\mathrm{NaBH}_{4}$ $(10 \mathrm{mmol})$ in absolute $\mathrm{EtOH}(10 \mathrm{~mL})$ under nitrogen atmosphere at room temperature for 1day. The mixture was poured into water. The resulting mixture was extracted with AcOEt (30 mL x 3) and dried over $\mathrm{MgSO}_{4}$. After filtration, the filtrate was concentrated under reduced pressure, and the residue was purified by flash silica gel column chromatography.

\section{General procedure for the phosphoric acid catalyzed kinetic resolution of acyclic amines and characterization data}

A typical procedure for the reaction of $r a c-\mathbf{1 b}$ is described.

A magnetic stirrer bar and $5 \AA$ MS $(50 \mathrm{mg})$ were placed in a round-bottom flask $(10 \mathrm{~mL})$ under nitrogen atmosphere. The $5 \AA$ MS were then dried with a heat gun under reduced pressure and the test-tube was refilled with nitrogen. $r a c-1 \mathbf{b}(22.7 \mathrm{mg}, 0.100 \mathrm{mmol})$, phosphoric acid $(R)$-TRIP (7.5 mg, $10 \mu \mathrm{mol})$, and imine 3a (54.2 $\mathrm{mg}, 0.200 \mathrm{mmol}$ ) were added to the round-bottom flask successively under nitrogen atmosphere at room temperature. Toluene, which was degassed by sonication under reduced pressure $(1 \mathrm{~mL})$, was added to the round-bottom flask. After being stirred for 2 days at $110{ }^{\circ} \mathrm{C}$ in oil bath, the mixture was filtered through Celite (washed with $\mathrm{CH}_{2} \mathrm{Cl}_{2}$ ) and the filtrate was concentrated under reduced pressure, and the residue was purified by preparative thin layer chromatography on silica gel (AcOEt $/$ hexane $=1 / 10$, twice) to give $10.9 \mathrm{mg}$ $(0.0480 \mathrm{mmol}, 48 \%)$ of $(S)-\mathbf{1 b}$ as a yellow oil.<smiles>CC(NC(C)(P)c1ccccc1)c1ccccc1</smiles>

(S)-1a $(40 \%, 91 \% \text { ee) [Table } 1 \text {, entry } 2]^{2}$

${ }^{1} \mathrm{H}$ NMR $\left(400 \mathrm{MHz}, \mathrm{CDCl}_{3}\right) \delta: 1.51(\mathrm{~d}, J=6.8 \mathrm{~Hz}, 3 \mathrm{H}) 3.68(\mathrm{~s}, 6 \mathrm{H}), 3.71(\mathrm{~s}, 3 \mathrm{H}), 4.43$ $(\mathrm{q}, J=6.8 \mathrm{~Hz}, 1 \mathrm{H}), 5.76(\mathrm{~s}, 2 \mathrm{H}), 7.20-7.27(\mathrm{~m}, 1 \mathrm{H}), 7.29-7.35(\mathrm{~m}, 2 \mathrm{H}), 7.35-7.39$ (m, 
$2 \mathrm{H})$.

${ }^{13} \mathrm{C} \mathrm{NMR}\left(100 \mathrm{MHz}, \mathrm{CDCl}_{3}\right) \delta: 24.9,54.1,55.6,60.9,90.8,125.7,126.9,128.6,129.7$, 144.0, 145.3, 153.6.

HPLC conditions: Daicel CHIRALCEL ${ }^{\circledR}$ AD-H column, hexane/2-propanol = 3/1, flow rate $=1.0 \mathrm{~mL} \mathrm{\operatorname {min } ^ { - 1 }}$, major enantiomer: $t_{\mathrm{R}}=7.61 \mathrm{~min}\left(\right.$ rac $\left.-1 \mathbf{a}, \mathrm{t}_{\mathrm{R}}=7.53 \mathrm{~min}\right)$; minor enantiomer: $\mathrm{t}_{\mathrm{R}}=15.64\left(\mathrm{rac}-\mathbf{1 a}, \mathrm{t}_{\mathrm{R}}=15.41 \mathrm{~min}\right)$.

$[\alpha]_{\mathrm{D}}^{25}+19.3\left(\mathrm{c} 1.0, \mathrm{CHCl}_{3}\right)\left[\right.$ lit., $[\alpha]_{\mathrm{D}}^{25}-20.7\left(\mathrm{c} 1.0, \mathrm{CHCl}_{3}\right)$ for $>99 \%$ ee of the

$(R)$-enantiomer $\left.{ }^{2}\right]$<smiles>CC(N=P)c1ccccc1</smiles>

(S)-1b $(48 \%, 97 \% \text { ee) [Table } 1 \text {, entry } 5]^{2}$

${ }^{1} \mathrm{H}$ NMR (400 MHz, $\left.\mathrm{CDCl}_{3}\right) \delta: 1.51(\mathrm{~d}, J=6.8 \mathrm{~Hz}, 3 \mathrm{H}), 3.70(\mathrm{~s}, 3 \mathrm{H}), 4.41(\mathrm{q}, J=6.8$ $\mathrm{Hz}, 1 \mathrm{H}), 6.46-6.51(\mathrm{~m}, 2 \mathrm{H}), 6.67-6.72(\mathrm{~m}, 2 \mathrm{H}), 7.20-7.25(\mathrm{~m}, 1 \mathrm{H}), 7.28-7.34(\mathrm{~m}, 2 \mathrm{H})$, 7.30-7.38 (m ,2H).

${ }^{13} \mathrm{C} \mathrm{NMR}\left(100 \mathrm{MHz}, \mathrm{CDCl}_{3}\right) \delta: 25.1,54.2,55.7,114.5,114.7,125.8,126.8,128.6$, 141.5, 145.4, 151.8 .

HPLC conditions: Daicel CHIRALCEL ${ }^{\circledR}$ OD-H column, hexane/2-propanol = 95/5, flow rate $=1.0 \mathrm{~mL} \mathrm{~min}{ }^{-1}$, major enantiomer: $\mathrm{t}_{\mathrm{R}}=10.12 \mathrm{~min}\left(\mathrm{rac}-\mathbf{1 b}, \mathrm{t}_{\mathrm{R}}=10.31 \mathrm{~min}\right)$; minor enantiomer: $t_{R}=9.49\left(\right.$ rac $\left.-1 \mathbf{b}, t_{R}=9.60 \mathrm{~min}\right)$.

$[\alpha]_{\mathrm{D}}^{25}-5.1\left(\mathrm{c} 1.0, \mathrm{CHCl}_{3}\right)\left[\right.$ lit., $[\alpha]_{\mathrm{D}}{ }^{25}+3.2\left(\mathrm{c} 1.0, \mathrm{CHCl}_{3}\right)$ for $91 \%$ ee of the

$(R)$-enantiomer $\left.{ }^{2}\right]$

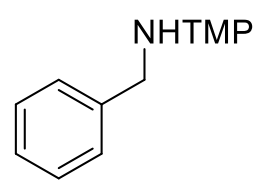

2a

${ }^{1} \mathrm{H} \mathrm{NMR}\left(400 \mathrm{MHz}, \mathrm{CDCl}_{3}\right)$ \&: 3.76 (s, 3H), 3.78 (s, 6H), 4.29 (s, 2H), 5.88 (s, 2H), 7.25-7.39 (m, 5H).

${ }^{13} \mathrm{C} \mathrm{NMR}\left(100 \mathrm{MHz}, \mathrm{CDCl}_{3}\right) \delta: 48.9,55.8,61.0,90.4,127.3,127.6,128.6,130.2,139.2$, 144.8, 153.9 . 
IR (film): 3394, 1611, 1510, 1235, $1129 \mathrm{~cm}^{-1}$.

HRMS (ESI) $m / z$ calcd for $\mathrm{C}_{16} \mathrm{H}_{19} \mathrm{NO}_{3}(\mathrm{M})^{+} 273.1365$, found 273.1363 .

m. p. $103-104{ }^{\circ} \mathrm{C}$.

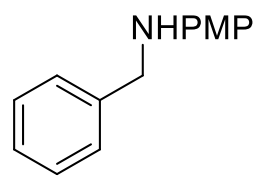

$\mathbf{2} \mathbf{b}^{2}$

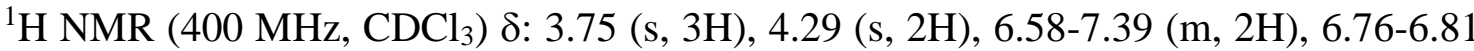
(m, 2H), 7.25-7.30 (m, 1H), 7.31-7.40 (m, 4H).

${ }^{13} \mathrm{C}$ NMR $\left(100 \mathrm{MHz}, \mathrm{CDCl}_{3}\right) \delta: 49.2,55.8,114.1,114.9,127.1,127.5,128.6,139.6$, $142.4,152.1$.<smiles>C[C@H](N=PN)c1ccc(-c2ccccc2)cc1</smiles>

$(S)-\mathbf{1} \mathbf{c}^{3}(37 \%, 91 \%$ ee $)$

${ }^{1} \mathrm{H}$ NMR (400 MHz, $\mathrm{CDCl}_{3}$ ) $\delta: 1.54(\mathrm{~d}, J=6.8 \mathrm{~Hz}, 3 \mathrm{H}), 3.69(\mathrm{~s}, 3 \mathrm{H}), 4.45$ (q, $J=6.8$ $\mathrm{Hz}, 1 \mathrm{H}), 6.50-6.55(\mathrm{~m}, 2 \mathrm{H}), 6.67-6.73(\mathrm{~m}, 2 \mathrm{H}), 7.29-7.34(\mathrm{~m}, 1 \mathrm{H}), 7.38-7.45(\mathrm{~m}, 4 \mathrm{H})$, 7.51-7.59 (m, 4H).

${ }^{13} \mathrm{C} \mathrm{NMR}\left(100 \mathrm{MHz}, \mathrm{CDCl}_{3}\right) \delta: 24.9,54.3,55.7,114.7,115.0,126.4,127.0,127.1$, $127.3,128.7,139.8,140.9,141.7,144.2,152.2$.

HPLC conditions: Daicel CHIRALCEL ${ }^{\circledR}$ OD-H column, hexane/2-propanol $=98 / 2$, flow rate $=0.6 \mathrm{~mL} \mathrm{m^{-1 }}$, major enantiomer: $t_{R}=18.19 \min \left(r a c-1 c, t_{R}=20.99 \mathrm{~min}\right)$; minor enantiomer: $t_{\mathrm{R}}=18.19 \mathrm{~min}\left(\mathrm{rac}-\mathbf{1 c}, \mathrm{t}_{\mathrm{R}}=19.23 \mathrm{~min}\right)$. $[\alpha]_{\mathrm{D}}^{25}-43.8\left(\mathrm{c} 0.5, \mathrm{CHCl}_{3}\right)\left[\right.$ lit., $[\alpha]_{\mathrm{D}}^{25}+41.0\left(\mathrm{c} 0.96, \mathrm{CHCl}_{3}\right)$ for $61 \%$ ee of the $(R)$-enantiomer $\left.{ }^{3}\right]$ 
<smiles>COc1ccc([C@H](C)NCPN)cc1OC</smiles>

$(S)-\mathbf{1 d}^{3}(50 \%, 93 \%$ ee $)$

${ }^{1} \mathrm{H}$ NMR (400 MHz, $\left.\mathrm{CDCl}_{3}\right) \delta: 1.49$ (d, $\left.J=6.8 \mathrm{~Hz}, 3 \mathrm{H}\right), 3.70(\mathrm{~s}, 3 \mathrm{H}), 3.849$ (s, 3H), $3.854(\mathrm{~s}, 3 \mathrm{H}), 4.35(\mathrm{q}, J=6.8 \mathrm{~Hz}, 1 \mathrm{H}), 6.48-6.52(\mathrm{~m}, 2 \mathrm{H}), 6.67-6.73(\mathrm{~m}, 2 \mathrm{H}), 6.79-6.83$ (m, 1H), 6.88-6.9 (m, 2H).

${ }^{13} \mathrm{C} \mathrm{NMR}\left(100 \mathrm{MHz}, \mathrm{CDCl}_{3}\right) \delta: 24.9,54.4,55.7,55.8,109.1,111.2,114.7,114.9,117.8$, $137.9,141.2,147.8,149.1,152.1$

HPLC conditions: Daicel CHIRALCEL ${ }^{\circledR}$ OD-H column, hexane/2-propanol $=90 / 10$ $\left(\mathrm{Et}_{2} \mathrm{NH}(0.5)\right.$ was used as additive. $)$, flow rate $=1.0 \mathrm{~mL} \mathrm{~min}^{-1}$, major enantiomer: $\mathrm{t}_{\mathrm{R}}=$ $11.77 \mathrm{~min}\left(\mathrm{rac}-\mathbf{1 d}, \mathrm{t}_{\mathrm{R}}=11.82 \mathrm{~min}\right)$; minor enantiomer: $10.82 \mathrm{~min}\left(\mathrm{rac}-\mathbf{1 d}, \mathrm{t}_{\mathrm{R}}=10.79\right.$ $\min )$.

$[\alpha]_{\mathrm{D}}^{25}-14.6\left(\mathrm{c} 0.5, \mathrm{CHCl}_{3}\right)\left[\right.$ lit., $[\alpha]_{\mathrm{D}}^{25}+8.3\left(\mathrm{c} 0.98, \mathrm{CHCl}_{3}\right)$ for $78 \%$ ee of the $(R)$-enantiomer $\left.{ }^{3}\right]$<smiles>Cc1cccc(C(C)NCPN)c1</smiles>

$(S)-1 \mathbf{e}^{3}(49 \%, 94 \%$ ee $)$

${ }^{1} \mathrm{H}$ NMR (400 MHz, $\mathrm{CDCl}_{3}$ ) $\delta: 1.49(\mathrm{~d}, J=6.8 \mathrm{~Hz}, 3 \mathrm{H}), 2.35$ (s, 3H), $3.71(\mathrm{~s}, 3 \mathrm{H}), 4.38$ $(\mathrm{q}, J=6.8 \mathrm{~Hz}, 1 \mathrm{H}), 6.46-6.53(\mathrm{~m}, 2 \mathrm{H}), 6.66-6.73(\mathrm{~m}, 2 \mathrm{H}), 7.01-7.07(\mathrm{~m}, 1 \mathrm{H}), 7.12-7.24$ (m, 4H), 7.51-7.59 (m, 3H).

${ }^{13} \mathrm{C} \mathrm{NMR}\left(100 \mathrm{MHz}, \mathrm{CDCl}_{3}\right) \delta: 21.5,25.1,54.3,55.7,114.5,114.7,122.9,126.6,127.6$, 128.5, 138.1, 141.7, 145.5, 151.8 .

HPLC conditions: Daicel CHIRALCEL ${ }^{\circledR}$ OD-H column, hexane/2-propanol $=98 / 2$, flow rate $=0.5 \mathrm{~mL} \mathrm{~min}{ }^{-1}$, major enantiomer: $t_{R}=20.43 \min \left(\right.$ rac $\left.-1 \mathbf{e}, t_{R}=20.43 \mathrm{~min}\right)$; minor enantiomer: $t_{R}=17.83\left(\right.$ rac-1e, $\left.t_{R}=17.72 \mathrm{~min}\right)$.

$[\alpha]_{\mathrm{D}}^{25}-11.6\left(\mathrm{c} 0.5, \mathrm{CHCl}_{3}\right)$ [lit., $[\alpha]_{\mathrm{D}}^{25}+2.5\left(\mathrm{c} 0.98, \mathrm{CHCl}_{3}\right)$ for $68 \%$ ee of the $(R)$-enantiomer $\left.{ }^{3}\right]$ 


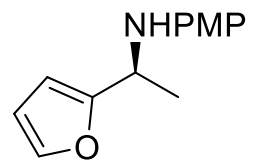

$(S)-\mathbf{1 f}^{4}(57 \%, 75 \%$ ee $)$

${ }^{1} \mathrm{H}$ NMR (400 MHz, $\left.\mathrm{CDCl}_{3}\right) \delta: 1.54(\mathrm{~d}, J=6.8 \mathrm{~Hz}, 3 \mathrm{H}), 3.74(\mathrm{~s}, 3 \mathrm{H}), 4.56(\mathrm{q}, J=6.8$ $\mathrm{Hz}, 1 \mathrm{H}), 6.12-6.17(\mathrm{~m}, 1 \mathrm{H}), 6.26-6.31(\mathrm{~m}, 1 \mathrm{H}), 6.57-6.65(\mathrm{~m}, 2 \mathrm{H}), 6.76-6.80(\mathrm{~m}, 2 \mathrm{H})$, 7.23-7.35 (m, 1H).

${ }^{13} \mathrm{C}$ NMR (100 MHz, $\left.\mathrm{CDCl}_{3}\right)$ \&: 20.9, 48.3, 55.6, 105.0, 110.0, 114.7, 115.1, 141.1, 141.3, 152.3, 157.4.

HPLC conditions: Daicel CHIRALCEL ${ }^{\circledR}$ OJ-H column, hexane/2-propanol = 10/1, flow rate $=1.0 \mathrm{~mL} \mathrm{~min}{ }^{-1}$, major enantiomer: $t_{\mathrm{R}}=38.13 \mathrm{~min}\left(\right.$ rac $\left.-\mathbf{2} \mathbf{e}, \mathrm{t}_{\mathrm{R}}=38.8 \mathrm{~min}\right)$; minor enantiomer: $\mathrm{t}_{\mathrm{R}}=43.41\left(\mathrm{rac}-\mathbf{2 e}, \mathrm{t}_{\mathrm{R}}=43.02 \mathrm{~min}\right)$.

$[\alpha]_{\mathrm{D}}^{25}-46.7$ (c 1.0, $\mathrm{CHCl}_{3}$ ) [lit., $[\alpha]_{\mathrm{D}}{ }^{25}-46.0\left(\mathrm{c} 1.0, \mathrm{CHCl}_{3}\right.$ ) for $62 \%$ ee of the $(S)$-enantiomer $\left.{ }^{4}\right]$

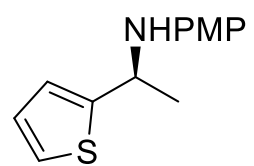

$(S)-\mathbf{1 g}^{4}(43 \%, 92 \%$ ee $)$

${ }^{1} \mathrm{H}$ NMR (400 MHz, $\mathrm{CDCl}_{3}$ ) $\delta: 1.62$ (d, $\left.J=6.8 \mathrm{~Hz}, 3 \mathrm{H}\right), 3.74(\mathrm{~s}, 3 \mathrm{H}), 4.76$ (q, $J=6.8$ $\mathrm{Hz}, 1 \mathrm{H}), 6.61(\mathrm{~d}, J=8.8 \mathrm{~Hz}, 2 \mathrm{H}), 6.77(\mathrm{~d}, J=8.8 \mathrm{~Hz}, 2 \mathrm{H}), 6.93-7.00(\mathrm{~m}, 2 \mathrm{H}), 7.15-7.18$ $(\mathrm{m}, 1 \mathrm{H})$.

${ }^{13} \mathrm{C}$ NMR $\left(100 \mathrm{MHz}, \mathrm{CDCl}_{3}\right) \delta: 24.8,50.5,55.7,114.8,115.1,122.9,123.6,126.7$, 141.2, 150.6, 152.4 .

HPLC conditions: Daicel CHIRALCEL ${ }^{\circledR}$ OD-H column, hexane/2-propanol $=20 / 1$, flow rate $=0.5 \mathrm{~mL} \mathrm{~min}{ }^{-1}$, major enantiomer: $\mathrm{t}_{\mathrm{R}}=24.06 \mathrm{~min}\left(\mathrm{rac}-\mathbf{1 g}, \mathrm{t}_{\mathrm{R}}=24.27 \mathrm{~min}\right)$; minor enantiomer: $t_{R}=21.99\left(\right.$ rac $\left.-1 \mathrm{~g}, t_{R}=22.11 \mathrm{~min}\right)$.

$[\alpha]_{\mathrm{D}}^{25}-7.9$ (c 1.0, $\left.\mathrm{CHCl}_{3}\right)$ [lit., $[\alpha]_{\mathrm{D}}{ }^{25}-9.0\left(\mathrm{c} 1.0, \mathrm{CHCl}_{3}\right)$ for $89 \%$ ee of the (S)-enantiomer $\left.{ }^{4}\right]$ 


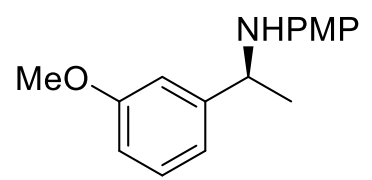

$(S)-\mathbf{1 h} \mathbf{h}^{3}(44 \%, 93 \% \text { ee })^{5}$

${ }^{1} \mathrm{H}$ NMR (400 MHz, $\mathrm{CDCl}_{3}$ ) $\delta: 1.50$ (d, $\left.J=6.8 \mathrm{~Hz}, 3 \mathrm{H}\right), 3.70(\mathrm{~s}, 3 \mathrm{H}), 3.79$ (s, 3H), 4.38 $(\mathrm{q}, J=6.8 \mathrm{~Hz}, 1 \mathrm{H}), 6.46-6.52(\mathrm{~m}, 2 \mathrm{H}), 6.66-6.71(\mathrm{~m}, 2 \mathrm{H}), 6.73-6.78(\mathrm{~m}, 1 \mathrm{H}), 6.95-7.00$ (m, 2H), 7.19-7.28 (m, 2H).

${ }^{13} \mathrm{C}$ NMR (100 MHz, $\left.\mathrm{CDCl}_{3}\right)$ \&: 25.0, 54.4, 55.1, 55.7, 111.7, 112.0, 114.65, 114.70, $118.2,129.6,130.2,141.4,147.2,152.0,159.9$.

HPLC conditions: Daicel CHIRALCEL ${ }^{\circledR}$ OD-H column, hexane/2-propanol $=95 / 5$, flow rate $=0.5 \mathrm{~mL} \mathrm{~min}^{-1}$, major enantiomer: $\mathrm{t}_{\mathrm{R}}=26.62 \mathrm{~min}\left(\mathrm{rac}-\mathbf{1} \mathbf{h}, \mathrm{t}_{\mathrm{R}}=27.09 \mathrm{~min}\right)$; minor enantiomer: $\mathrm{t}_{\mathrm{R}}=23.61\left(\mathrm{rac}-\mathbf{1 h}, \mathrm{t}_{\mathrm{R}}=23.83 \mathrm{~min}\right)$.

$[\alpha]_{\mathrm{D}}^{25}-10.6\left(\mathrm{c} 0.5, \mathrm{CHCl}_{3}\right)$ [lit., $[\alpha]_{\mathrm{D}}^{25}+5.5\left(\mathrm{c} 1.05, \mathrm{CHCl}_{3}\right)$ for $72 \%$ ee of the $(R)$-enantiomer $\left.{ }^{3}\right]$<smiles>C[C@H](NCPN)c1ccc(F)cc1</smiles>

$(S)-1 i^{5}(46 \%, 92 \%$ ee) (43\% yield, 1 mmol scale experiment)

Procedure for $1 \mathrm{mmol}$ scale experiment: A magnetic stirrer bar and $5 \AA \mathrm{MS}$ (500 mg) were placed in a round-bottom flask $(30 \mathrm{~mL})$ under nitrogen atmosphere. The $5 \AA \mathrm{MS}$ were then dried with a heat gun under reduced pressure and the test-tube was refilled with nitrogen. rac-1i (245 mg, $1.00 \mathrm{mmol}),(R)$-TRIP (75.3 mg, $0.100 \mathrm{mmol})$, and 3a (542 mg, $2.00 \mathrm{mmol}$ ) were added to the round-bottom flask successively under nitrogen atmosphere at room temperature. Degassed toluene $(10 \mathrm{~mL})$, was added to the round-bottom flask. After being stirred for 7 days at $110{ }^{\circ} \mathrm{C}$ in oil bath, the mixture was filtered through Celite (washed with $\mathrm{CH}_{2} \mathrm{Cl}_{2}$ ) and the filtrate was concentrated under reduced pressure, and the residue was purified by preparative thin layer chromatography on silica gel (AcOEt / hexane $=1 / 10$, three times) to give $106 \mathrm{mg}(0.432 \mathrm{mmol}, 43 \%$, $92 \%$ ee)) of $(S)-\mathbf{1 i}$ as a pale yellow oil.

${ }^{1} \mathrm{H}$ NMR (400 MHz, $\left.\mathrm{CDCl}_{3}\right) \delta: 1.49(\mathrm{~d}, J=6.8 \mathrm{~Hz}, 3 \mathrm{H}), 3.70(\mathrm{~s}, 3 \mathrm{H}), 4.40$ (q, $J=6.8$ $\mathrm{Hz}, 1 \mathrm{H}), 6.43-6.49$ (m, 2H), 6.66-6.73 (m, 2H), 6.96-7.02 (m, H), 7.30-7.36 (m, 2H). 
${ }^{13} \mathrm{C}$ NMR $\left(100 \mathrm{MHz}, \mathrm{CDCl}_{3}\right) \delta: 25.1,53.8,55.7,114.7,115.4(\mathrm{~d}, J=20.8 \mathrm{~Hz}), 127.4(\mathrm{~d}$, $J=8.1 \mathrm{~Hz}), 114.70,141.0(\mathrm{~d}, J=3.1 \mathrm{~Hz}), 152.1,161.7(\mathrm{~d}, \mathrm{~J}=243.3 \mathrm{~Hz})$.

HPLC conditions: Daicel CHIRALCEL ${ }^{\circledR}$ OD-H column, hexane/2-propanol $=95 / 5$, flow rate $=0.5 \mathrm{~mL} \mathrm{\operatorname {min } ^ { - 1 }}$, major enantiomer: $\mathrm{t}_{\mathrm{R}}=22.54 \mathrm{~min}\left(\right.$ rac $\left.-\mathbf{1 i}, \mathrm{t}_{\mathrm{R}}=22.79 \mathrm{~min}\right)$; minor enantiomer: $\mathrm{t}_{\mathrm{R}}=20.83\left(\mathrm{rac}-\mathbf{1 i}, \mathrm{t}_{\mathrm{R}}=20.87 \mathrm{~min}\right)$.

$[\alpha]_{\mathrm{D}}^{25}+11.8\left(\mathrm{c} 0.5, \mathrm{CHCl}_{3}\right)$ [lit., $[\alpha]_{\mathrm{D}}{ }^{25}-19.4\left(\mathrm{c} 0.3, \mathrm{CHCl}_{3}\right)$ for $94 \%$ ee of the $(R)$-enantiomer $]^{5}$<smiles>CC(NC(=O)NCc1ccccc1)c1ccc(I)cc1</smiles>

(S)-1j $(55 \%, 73 \%$ ee $)$

${ }^{1} \mathrm{H}$ NMR (400 MHz, $\left.\mathrm{CDCl}_{3}\right) \delta: 1.51(\mathrm{~d}, J=6.8 \mathrm{~Hz}, 3 \mathrm{H}), 3.70(\mathrm{~s}, 3 \mathrm{H}), 4.42(\mathrm{q}, J=6.8$ $\mathrm{Hz}, 1 \mathrm{H}), 6.46-6.52(\mathrm{~m}, 2 \mathrm{H}), 6.66-6.73(\mathrm{~m}, 2 \mathrm{H}), 7.19-7.40(\mathrm{~m}, 4 \mathrm{H})$.

${ }^{13} \mathrm{C} \mathrm{NMR}\left(100 \mathrm{MHz}, \mathrm{CDCl}_{3}\right) \delta: 25.0,54.3,55.7,114.6,114.7,125.9,126.8,128.6$, 141.4, 145.3, 151.9 .

IR (film): 3403, 2963, 2,831, 1510, 1236, $816 \mathrm{~cm}^{-1}$.

HRMS (ESI) $m / z$ calcd for $\mathrm{C}_{15} \mathrm{H}_{15} \mathrm{NOI}(\mathrm{M})^{+} 352.0199$, found 352.0194.

HPLC conditions: Daicel CHIRALCEL ${ }^{\circledR}$ OD-H column, hexane/2-propanol $=95 / 5$, flow rate $=0.5 \mathrm{~mL} \mathrm{\operatorname {min } ^ { - 1 }}$, major enantiomer: $\mathrm{t}_{\mathrm{R}}=20.12 \mathrm{~min}\left(\mathrm{rac}-\mathbf{1} \mathbf{j}, \mathrm{t}_{\mathrm{R}}=20.46 \mathrm{~min}\right)$; minor enantiomer: $\mathrm{t}^{\mathrm{R}}=18.55\left(\right.$ rac $\left.\mathbf{- 1} \mathbf{j}, \mathrm{t}_{\mathrm{R}}=18.75 \mathrm{~min}\right)$.

$[\alpha]_{\mathrm{D}}^{25}-3.5\left(\mathrm{c} 1.0, \mathrm{CHCl}_{3}\right)$<smiles>C[C@H](NCPN)c1ccc(Br)cc1</smiles>

$(S)-\mathbf{1} \mathbf{k}^{3}(49 \%, 97 \%$ ee $)$

${ }^{1} \mathrm{H}$ NMR (400 MHz, $\left.\mathrm{CDCl}_{3}\right) \delta: 1.48(\mathrm{~d}, J=6.8 \mathrm{~Hz}, 3 \mathrm{H}), 3.70(\mathrm{~s}, 3 \mathrm{H}), 4.37$ (q, $J=6.8$ $\mathrm{Hz}, 1 \mathrm{H}), 6.41-6.47$ (m, 2H), 6.67-6.73 (m, 2H), $7.15(\mathrm{~d}, J=8.0 \mathrm{~Hz}, 2 \mathrm{H}), 7.43$ (d, $J=8.0$ $\mathrm{Hz}, 2 \mathrm{H})$.

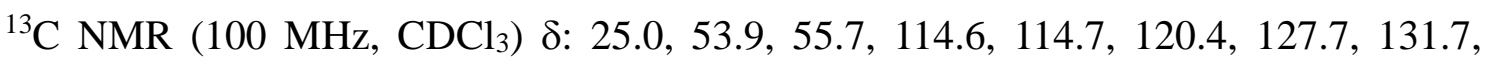
$141.0,144.5,152.1$. 
HPLC conditions: Daicel CHIRALCEL ${ }^{\circledR}$ OD-H column, hexane/2-propanol = 98/2, flow rate $=0.5 \mathrm{~mL} \mathrm{~min}{ }^{-1}$, major enantiomer: $\mathrm{t}_{\mathrm{R}}=40.03 \mathrm{~min}\left(\right.$ rac $\left.-\mathbf{1 k}, \mathrm{t}_{\mathrm{R}}=40.34 \mathrm{~min}\right)$; minor enantiomer: $\mathrm{t}^{\mathrm{R}}=32.95\left(\right.$ rac $\left.-1 \mathbf{k}, \mathrm{t}_{\mathrm{R}}=32.55 \mathrm{~min}\right)$.

$[\alpha]_{\mathrm{D}}^{25}-17.1\left(\mathrm{c} 1.0, \mathrm{CHCl}_{3}\right)$ [lit., $[\alpha]_{\mathrm{D}}^{25}+13.5\left(\mathrm{c} 1.10, \mathrm{CHCl}_{3}\right)$ for $59 \%$ ee of the $(R)$-enantiomer $]^{3}$

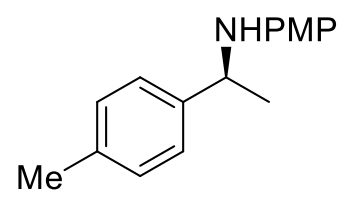

$(S)-11^{3}(45 \%, 94 \%$ ee $)$

${ }^{1} \mathrm{H}$ NMR $\left(400 \mathrm{MHz}, \mathrm{CDCl}_{3}\right) \delta: 1.47(\mathrm{~d}, J=6.8 \mathrm{~Hz}, 3 \mathrm{H}), 2.31(\mathrm{~s}, 3 \mathrm{H}), 3.68(\mathrm{~s}, 3 \mathrm{H}), 4.38$ $(\mathrm{q}, J=6.8 \mathrm{~Hz}, 1 \mathrm{H}), 6.45-6.49(\mathrm{~m}, 2 \mathrm{H}), 6.66-6.71(\mathrm{~m}, 2 \mathrm{H}), 7.11(\mathrm{~d}, J=8.0 \mathrm{~Hz}, 2 \mathrm{H}), 7$. $24(\mathrm{~d}, J=8.0 \mathrm{~Hz}, 2 \mathrm{H})$.

${ }^{13} \mathrm{C} \mathrm{NMR}\left(100 \mathrm{MHz}, \mathrm{CDCl}_{3}\right) \delta: 21.0,25.1,53.9,55.7,114.5,114.7,125.8,129.3,136.3$, 141.6, 142.4, 151.8 .

HPLC conditions: Daicel CHIRALCEL ${ }^{\circledR}$ OD-H column, hexane/2-propanol $=98 / 2$, flow rate $=1.0 \mathrm{~mL} \mathrm{~min}{ }^{-1}$, major enantiomer: $\mathrm{t}_{\mathrm{R}}=10.58 \mathrm{~min}\left(\mathrm{rac}-\mathbf{1 1}, \mathrm{t}_{\mathrm{R}}=10.79 \mathrm{~min}\right)$; minor enantiomer: $\mathrm{t}^{\mathrm{R}}=9.31 \mathrm{~min}\left(\mathrm{rac}-\mathbf{1 1}, \mathrm{t}_{\mathrm{R}}=9.53 \mathrm{~min}\right)$.

$[\alpha]_{\mathrm{D}}^{25}-14.2$ (c 1.0, $\mathrm{CHCl}_{3}$ ) [lit., $[\alpha]_{\mathrm{D}}{ }^{25}+11.5$ (c 1.12, $\mathrm{CHCl}_{3}$ ) for $61 \%$ ee of the $(R)$-enantiomer $]^{3}$

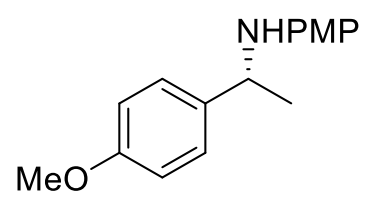

$(R)-\mathbf{1 m}^{3}(48 \%, 95$ ee) This reaction was carried out using $(\boldsymbol{S})$-TRIP catalyst.

${ }^{1} \mathrm{H}$ NMR $\left(400 \mathrm{MHz}, \mathrm{CDCl}_{3}\right) \delta: 1.46(\mathrm{~d}, J=6.4 \mathrm{~Hz}, 3 \mathrm{H}), 3.68(\mathrm{~s}, 3 \mathrm{H}), 3.76(\mathrm{~s}, 3 \mathrm{H}), 4.36$ $(\mathrm{q}, J=6.4 \mathrm{~Hz}, 1 \mathrm{H}), 6.44-6.49(\mathrm{~m}, 2 \mathrm{H}), 6.66-6.71(\mathrm{~m}, 2 \mathrm{H}), 6.81-6.86(\mathrm{~m}, 2 \mathrm{H}), 7.24(\mathrm{~m}$, $2 \mathrm{H})$.

${ }^{13} \mathrm{C} \mathrm{NMR}\left(100 \mathrm{MHz}, \mathrm{CDCl}_{3}\right) \delta: 25.0,53.6,55.2,55.7,113.9,114.6,114.7,126.1,137.4$, 141.5, 151.8, 158.4 .

HPLC conditions: Daicel CHIRALCEL ${ }^{\circledR}$ OD-H column, hexane/2-propanol $=20 / 1$, flow rate $=1.0 \mathrm{~mL} \mathrm{~min}{ }^{-1}$, major enantiomer: $\mathrm{t}_{\mathrm{R}}=10.73 \mathrm{~min}\left(\mathrm{rac}-\mathbf{1 m}, \mathrm{t}_{\mathrm{R}}=10.87 \mathrm{~min}\right)$; 
minor enantiomer: $t_{R}=12.54 \min \left(r a c-1 m, t_{R}=12.61 \mathrm{~min}\right)$.

$[\alpha]_{\mathrm{D}}^{25}+17.3\left(\mathrm{c} 1.0, \mathrm{CHCl}_{3}\right)$ [lit., $[\alpha]_{\mathrm{D}}^{25}+10.1\left(\mathrm{c} 1.0, \mathrm{CHCl}_{3}\right)$ for $65 \%$ ee of the $(R)$-enantiomer $]^{3}$

Me

$(S)-1 \mathbf{n}^{6}(52 \%, 86 \%$ ee $)$

${ }^{1} \mathrm{H}$ NMR (400 MHz, $\mathrm{CDCl}_{3}$ ) $\delta: 1.46(\mathrm{~d}, J=6.8 \mathrm{~Hz}, 3 \mathrm{H}), 2.44$ (s, 3H), 3.70 (s, 3H), 3.77 (brs, 1H), 4.62 (q, $J=6.8 \mathrm{~Hz}, 1 \mathrm{H}), 6.38-6.45(\mathrm{~m}, 2 \mathrm{H}), 6.67-6.73(\mathrm{~m}, 2 \mathrm{H}), 7.11-7.19$ (m, $3 \mathrm{H}), 7.40-7.46(\mathrm{~m}, 1 \mathrm{H})$.

${ }^{13} \mathrm{C}$ NMR (100 MHz, $\left.\mathrm{CDCl}_{3}\right) \delta: 19.0,23.1,50.4,55.7,114.1,114.8,124.6,126.53$, $126.55,130.5,134.5,141.6,143.0,151.8$.

HPLC conditions: Daicel CHIRALCEL ${ }^{\circledR}$ OD-H column, hexane/2-propanol = 98/2, flow rate $=0.5 \mathrm{~mL} \mathrm{~min}{ }^{-1}$, major enantiomer: $t_{\mathrm{R}}=20.95 \mathrm{~min}\left(\mathrm{rac}-\mathbf{1 n}, \mathrm{t}_{\mathrm{R}}=21.03 \mathrm{~min}\right)$; minor enantiomer: $\mathrm{t}_{\mathrm{R}}=16.42\left(\mathrm{rac}-\mathbf{1 n}, \mathrm{t}_{\mathrm{R}}=16.37 \mathrm{~min}\right)$.

$[\alpha]_{\mathrm{D}}^{25}+6.8\left(\mathrm{c} 0.5, \mathrm{CHCl}_{3}\right)$ [lit., $[\alpha]_{\mathrm{D}}^{25}+9.2\left(\mathrm{c} 2.0, \mathrm{CHCl}_{3}\right)$ for $44 \%$ ee of the $(S)$-enantiomer $]^{6}$<smiles>CC(N=P)c1cccc2ccccc12</smiles>

$(R)-\mathbf{1 0}^{7}(45 \%, 88 \%$ ee) This reaction was carried out using $(\boldsymbol{S})$-TRIP catalyst.

${ }^{1} \mathrm{H}$ NMR (400 MHz, $\left.\mathrm{CDCl}_{3}\right) \delta: 1.69$ (d, $\left.J=6.8 \mathrm{~Hz}, 3 \mathrm{H}\right), 3.71$ (s, 3H), 4.01 (brs, 1H), $5.27(\mathrm{q}, J=6.8 \mathrm{~Hz}, 1 \mathrm{H}), 6.38-6.44(\mathrm{~m}, 2 \mathrm{H}), 6.61-6.66(\mathrm{~m}, 2 \mathrm{H}), 7.35-7.41(\mathrm{~m}, 1 \mathrm{H})$, 7.44-7.56 (m, 2H), 7.61-7.65 (m, 1H), 7.69-7.74 (m, 1H), 7.85-7.90 (m, 1H), 8.11-8.17 $(\mathrm{m}, 1 \mathrm{H})$.

${ }^{13} \mathrm{C}$ NMR $\left(100 \mathrm{MHz}, \mathrm{CDCl}_{3}\right) \delta: 23.7,50.1,55.6,114.3,114.7,122.3,122.5,125.3$, $125.8,126.0,127.3,129.1,130.7,134.0,140.1,141.2,151.8$.

HPLC conditions: Daicel CHIRALCEL ${ }^{\circledR}$ OD-H column, hexane/2-propanol = 5/1, flow rate $=1.0 \mathrm{~mL} \mathrm{~min}{ }^{-1}$, major enantiomer: $\mathrm{t}_{\mathrm{R}}=11.20 \mathrm{~min}\left(\mathrm{rac}-\mathbf{1 o}, \mathrm{t}_{\mathrm{R}}=11.27 \mathrm{~min}\right) ;$ minor 
enantiomer: $\mathrm{t}_{\mathrm{R}}=16.47\left(\mathrm{rac}-\mathbf{1 0}, \mathrm{t}_{\mathrm{R}}=16.59 \mathrm{~min}\right)$.

$[\alpha]_{\mathrm{D}}^{25}-90.8\left(\mathrm{c} 0.5, \mathrm{CHCl}_{3}\right)$ [lit., $[\alpha]_{\mathrm{D}}^{23}-158.4\left(\mathrm{c} 0.9, \mathrm{CHCl}_{3}\right)$ for $84 \%$ ee of the $(R)$-enantiomer $]^{7}$

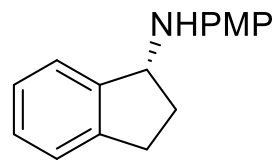

$(R)-1 \mathbf{p}^{5}(52 \%, 70 \%$ ee) This reaction was carried out using $(\boldsymbol{S})$-TRIP catalyst.

${ }^{1} \mathrm{H}$ NMR $\left(400 \mathrm{MHz}, \mathrm{CDCl}_{3}\right) \delta: 1.83-1.95(\mathrm{~m}, 1 \mathrm{H}), 2.53-2.65(\mathrm{~m}, 1 \mathrm{H}), 2.85-2.96(\mathrm{~m}$, $1 \mathrm{H}), 2.97-3.11(\mathrm{~m}, 1 \mathrm{H}), 3.78(\mathrm{~s}, 3 \mathrm{H}), 4.96(\mathrm{t}, J=6.4 \mathrm{~Hz}, 1 \mathrm{H}), 6.68-6.74(\mathrm{~m}, 2 \mathrm{H}), 6.79$ $-6.85(\mathrm{~m}, 2 \mathrm{H}), 7.19-7.29(\mathrm{~m}, 3 \mathrm{H}), 7.36-7.41(\mathrm{~m}, 1 \mathrm{H})$.

${ }^{13} \mathrm{C}$ NMR (100 MHz, $\left.\mathrm{CDCl}_{3}\right) \delta:$ 30.2, 33.8, 55.8, 59.6, 114.8, 115.0, 124.3, 124.8, 126.6, 126.9, 127.8, 143.6, 144.6, 152.2.

HPLC conditions: Daicel CHIRALPAK ${ }^{\circledR}$ IB column, hexane/2-propanol $=100 / 1$, flow rate $=0.3 \mathrm{~mL} \mathrm{~min}^{-1}$, major enantiomer: $\mathrm{t}_{\mathrm{R}}=21.89 \mathrm{~min}\left(\right.$ rac-1p, $\left.\mathrm{t}_{\mathrm{R}}=21.83 \mathrm{~min}\right)$; minor enantiomer: $\mathrm{t}_{\mathrm{R}}=20.94 \mathrm{~min}\left(\mathrm{rac}-\mathbf{1} \mathbf{p}, \mathrm{t}_{\mathrm{R}}=20.83 \mathrm{~min}\right)$.

$[\alpha]_{\mathrm{D}}^{25}+17.0$ (c 1.0, $\left.\mathrm{CHCl}_{3}\right)$ [lit., $[\alpha]_{\mathrm{D}}{ }^{24}+45.4\left(\mathrm{c} 0.22, \mathrm{CHCl}_{3}\right)$ for $85 \%$ ee of the $(R)$-enantiomer $]^{5}$

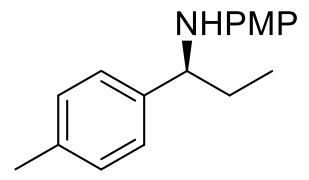

$(S)-1 q^{8}(55 \%, 67 \%$ ee $)$

${ }^{1} \mathrm{H}$ NMR $\quad\left(400 \mathrm{MHz}, \mathrm{CDCl}_{3}\right) \delta: 0.90-0.98(\mathrm{~m}, 3 \mathrm{H}), 1.73-1.86(\mathrm{~m}, 2 \mathrm{H}), 2.32(\mathrm{~s}, 3 \mathrm{H})$, $3.69(\mathrm{~s}, 3 \mathrm{H}), 4.12(\mathrm{t}, J=6.8 \mathrm{~Hz}, 1 \mathrm{H}), 6.45-6.51(\mathrm{~m}, 2 \mathrm{H}), 6.66-6.72(\mathrm{~m}, 2 \mathrm{H}), 7.09-7.15$ (m, 2H), $7.24-7.26(\mathrm{~m}, 2 \mathrm{H})$.

${ }^{13} \mathrm{C} \mathrm{NMR}\left(100 \mathrm{MHz}, \mathrm{CDCl}_{3}\right) \delta: 10.8,21.0,31.7,55.7,60.2,114.4,114.7,126.4,129.1$, 136.3, 141.1, 141.9, 151.7 .

HPLC conditions: Daicel CHIRALCEL ${ }^{\circledR}$ OD-H column, hexane/2-propanol = 98/2, flow rate $=1.0 \mathrm{~mL} \mathrm{~min}{ }^{-1}$, major enantiomer: $t_{R}=8.09 \min \left(\right.$ rac-1q, $\left.t_{R}=8.11 \mathrm{~min}\right)$; minor enantiomer: $t_{R}=7.43 \mathrm{~min}\left(r a c-1 q, t_{R}=7.43 \mathrm{~min}\right)$.

$[\alpha]_{\mathrm{D}}^{23}-23.8\left(\mathrm{c} 1.00, \mathrm{CHCl}_{3}\right)$ [lit., $[\alpha]_{\mathrm{D}}{ }^{23}+36.6\left(\mathrm{c} 1.05, \mathrm{CHCl}_{3}\right)$ for $94 \%$ ee of the $(R)$-enantiomer $]^{8}$ 
$\overbrace{}^{\mathrm{NHPMP}}$

$(S)-\mathbf{1} \mathbf{r}^{9}(54 \%, 86 \%$ ee $)$

${ }^{1} \mathrm{H}$ NMR $\left(400 \mathrm{MHz}, \mathrm{CDCl}_{3}\right) \delta: 1.21(\mathrm{~d}, \mathrm{~J}=6.4 \mathrm{~Hz}, 3 \mathrm{H}), 1.67-1.80(\mathrm{~m}, 1 \mathrm{H}), 1.82-1.91$ (m, 1H), 2.74 (dd, J = 8.4, 8.4 Hz, $2 \mathrm{H}$ ), 3.40 (ddq, J = 6.4, 6.4, 6.4 Hz, $1 \mathrm{H}$ ), 3.75 (s, $3 \mathrm{H}), 6.48-6.53(\mathrm{~m}, 2 \mathrm{H}), 6.72-6.78(\mathrm{~m}, 2 \mathrm{H}), 7.15-7.21(\mathrm{~m}, 3 \mathrm{H}), 7.24-7.31(\mathrm{~m}, 2$ $\mathrm{H})$.

${ }^{13} \mathrm{C} \mathrm{NMR}\left(100 \mathrm{MHz}, \mathrm{CDCl}_{3}\right) \delta: 20.8,32.5,38.8,48.9,55.8,114.7,114.9,125.8,128.29$, $128.34,128.4,142.1,151.8$.

HPLC conditions: Daicel CHIRALCEL ${ }^{\circledR}$ OD-H column, hexane/2-propanol $=20 / 1$, flow rate $=1.0 \mathrm{~mL} \mathrm{~min}{ }^{-1}$, major enantiomer: $t_{R}=12.54 \min \left(\right.$ rac-1r,$\left.t_{R}=12.93 \mathrm{~min}\right)$; minor enantiomer: $\mathrm{t}_{\mathrm{R}}=11.66 \mathrm{~min}\left(\mathrm{rac}-\mathbf{1} \mathbf{r}, \mathrm{t}_{\mathrm{R}}=11.81 \mathrm{~min}\right)$.

$[\alpha]_{\mathrm{D}}{ }^{24}-4.5\left(\mathrm{c} 1.0, \mathrm{CHCl}_{3}\right)$ [lit., $[\alpha]_{\mathrm{D}}{ }^{24}-7.0\left(\mathrm{c} 2.3, \mathrm{CHCl}_{3}\right)$ for $97 \%$ ee of the $(R)$-enantiomer $]^{9}$<smiles>CC(C)CC(C)NP=NP</smiles>

$(S)-1 \mathbf{s}^{9}(60 \%, 73 \%$ ee $)$

${ }^{1} \mathrm{H}$ NMR (400 MHz, $\mathrm{CDCl}_{3}$ ) $\delta: 0.91(\mathrm{~d}, J=6.4 \mathrm{~Hz}, 3 \mathrm{H}), 0.93(\mathrm{~d}, J=6.4 \mathrm{~Hz}, 3 \mathrm{H}), 1.13$ (d, $J=6.4 \mathrm{~Hz}, 3 \mathrm{H}), 1.19-1.27$ (m, $1 \mathrm{H}), 1.41-1.50$ (m, $1 \mathrm{H}), 1.75(\mathrm{dq}, J=6.8,6.4 \mathrm{~Hz}, 1$ H), 3.43 (ddq, $J=6.4,6.4,6.4 \mathrm{~Hz}, 1 \mathrm{H}), 3.74$ (s, $3 \mathrm{H}), 6.54-6.58(\mathrm{~m}, 2 \mathrm{H}), 6.74-6.79$ (m, $2 \mathrm{H})$.

${ }^{13} \mathrm{C}$ NMR (100 MHz, $\left.\mathrm{CDCl}_{3}\right) \delta:$ 21.0, 22.5, 23.0, 25.1, 46.9, 47.6, 55.8, 114.7, 114.9, 141.8, 151.8 .

HPLC conditions: Daicel CHIRALCEL ${ }^{\circledR}$ AS-H column, hexane/2-propanol = 100/1, flow rate $=1.0 \mathrm{~mL} \mathrm{~min}{ }^{-1}$, major enantiomer: $t_{R}=4.56 \min \left(\right.$ rac $\left.-1 \mathrm{~s}, t_{R}=4.62 \mathrm{~min}\right)$; minor enantiomer: $\mathrm{t}_{\mathrm{R}}=5.79 \mathrm{~min}\left(\mathrm{rac}-\mathbf{1 s}, \mathrm{t}_{\mathrm{R}}=5.79 \mathrm{~min}\right)$.

$[\alpha]_{\mathrm{D}}^{25}+8.0\left(\mathrm{c} 1.0, \mathrm{CHCl}_{3}\right)$ [lit., $[\alpha]_{\mathrm{D}}{ }^{24}-12.7\left(\mathrm{c} 1.1, \mathrm{CHCl}_{3}\right)$ for $97 \%$ ee of the $(R)$-enantiomer $]^{9}$

\section{Computational details}

All calculations were performed with the Gaussian 16 package. ${ }^{10}$ Geometries were fully optimized at the B3LYP/6-31G* level and characterized by frequency calculation. 
Based on our previous computational studies, ${ }^{2,8,11}$ we investigated four diastereomeric transition state (TS) models that form the dicoordinated cyclic TS structure and incorporate Z-ketimine. Other two diastereomeric TS models corresponding to transferable hydrogen atoms of 2a (TS'major and TS'minor) exhibit a similar tendency of energetic relationship and structural properties with TSmajor and TSminor (Figure S1). TS'major is more stable than TS'minor. The arrangement of amine and ketimine is significantly changed between TS'major and TS'minor. In TS'minor, to reduce the steric repulsion, both amine and ketimine are positioned far from the phosphoric acid moiety.
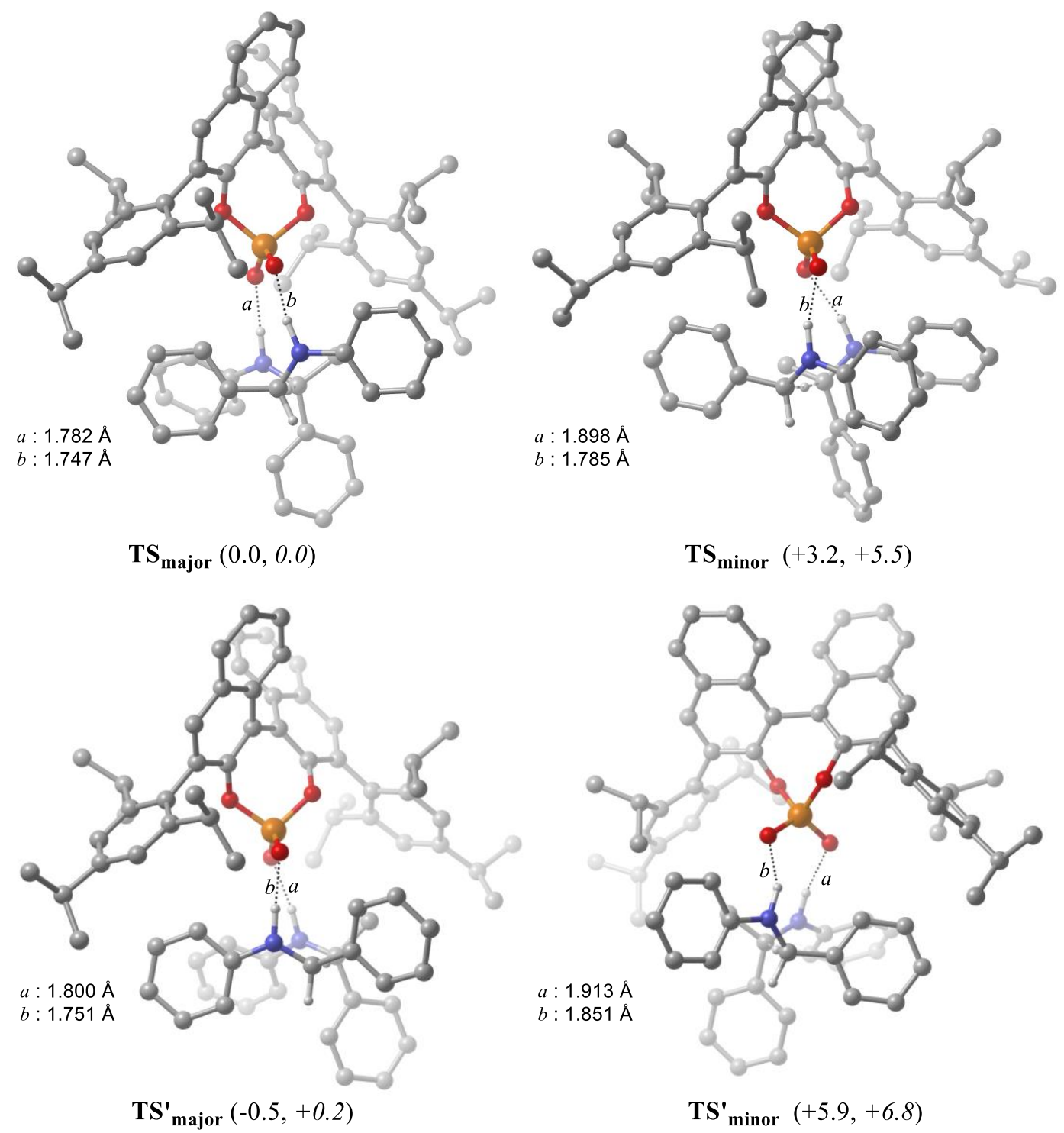

Figure S1. 3D structures and the relative energies ( $\mathrm{kcal} / \mathrm{mol}$, Gibbs free energies are in italics) of four diastereomeric TS models 


\section{Cartesian coordinates}

TSmajor

SCF Done: $E(R B 3 L Y P)=-3736.08970289 \mathrm{~A} \cdot \mathrm{U}$.

Sum of electronic and thermal Free Energies $=-3734.800264 \mathrm{~A}$.U.

Center Atomic Atomic Coordinates (Angstroms)

Number Number Type X $\quad$ Y Z

\begin{tabular}{|c|c|c|c|c|c|}
\hline 1 & 6 & 0 & 2.185833 & -3.007572 & 1.569873 \\
\hline 2 & 7 & 0 & 1.525806 & -2.082719 & 2.320565 \\
\hline 3 & 6 & 0 & 1.995135 & -1.506618 & 3.515073 \\
\hline 4 & 6 & 0 & 1.191609 & -0.516027 & 4.109170 \\
\hline 5 & 6 & 0 & 3.195115 & -1.885559 & 4.139445 \\
\hline 6 & 6 & 0 & 1.581303 & 0.069086 & 5.309614 \\
\hline 7 & 1 & 0 & 0.273930 & -0.216576 & 3.612218 \\
\hline 8 & 6 & 0 & 3.570823 & -1.288276 & 5.342436 \\
\hline 9 & 1 & 0 & 3.844769 & -2.636800 & 3.702375 \\
\hline 10 & 6 & 0 & 2.770238 & -0.312440 & 5.937017 \\
\hline 11 & 1 & 0 & 0.949275 & 0.832140 & 5.755135 \\
\hline 12 & 1 & 0 & 4.501104 & -1.592427 & 5.814771 \\
\hline 13 & 1 & 0 & 3.070476 & 0.147017 & 6.874166 \\
\hline 14 & 6 & 0 & 3.055926 & -2.000179 & -2.607623 \\
\hline 15 & 6 & 0 & 3.671513 & -1.501516 & -0.176188 \\
\hline 16 & 7 & 0 & 2.902291 & -1.431461 & -1.324534 \\
\hline 17 & 1 & 0 & 2.006781 & -0.927985 & -1.195510 \\
\hline 18 & 1 & 0 & 3.018132 & -2.314355 & 0.658960 \\
\hline 19 & 6 & 0 & 5.015706 & -2.191710 & -0.210892 \\
\hline 20 & 6 & 0 & 6.181438 & -1.514897 & 0.175226 \\
\hline 21 & 6 & 0 & 5.117211 & -3.544274 & -0.578660 \\
\hline 22 & 6 & 0 & 7.416277 & -2.167781 & 0.181405 \\
\hline 23 & 1 & 0 & 6.134012 & -0.470674 & 0.464712 \\
\hline 24 & 6 & 0 & 6.347188 & -4.196971 & -0.563785 \\
\hline 25 & 1 & 0 & 4.227930 & -4.082051 & -0.889044 \\
\hline 26 & 6 & 0 & 7.503705 & -3.510128 & -0.184577 \\
\hline 27 & 1 & 0 & 8.308400 & -1.622446 & 0.476888 \\
\hline 28 & 1 & 0 & 6.404025 & -5.242823 & -0.853168 \\
\hline 29 & 1 & 0 & 8.463756 & -4.018801 & -0.174880 \\
\hline 30 & 1 & 0 & 0.701306 & -1.601792 & 1.907845 \\
\hline 31 & 8 & 0 & 0.479708 & -0.022035 & -1.039120 \\
\hline 32 & 15 & 0 & -0.538129 & 0.266946 & 0.027572 \\
\hline 33 & 8 & 0 & -2.015359 & 0.195468 & -0.719255 \\
\hline 34 & 8 & 0 & -0.497965 & 1.853804 & 0.510377 \\
\hline 35 & 8 & 0 & -0.544756 & -0.534543 & 1.307911 \\
\hline 36 & 6 & 0 & -3.130908 & 0.710690 & -0.084916 \\
\hline 37 & 6 & 0 & -0.918002 & 2.872554 & -0.323333 \\
\hline 38 & 6 & 0 & -3.267490 & 2.088868 & 0.023322 \\
\hline 39 & 6 & 0 & -4.143185 & -0.200550 & 0.341706 \\
\hline 40 & 6 & 0 & -2.271290 & 2.997632 & -0.612784 \\
\hline 41 & 6 & 0 & 0.052734 & 3.825294 & -0.759723 \\
\hline 42 & 6 & 0 & -5.264727 & 0.329996 & 0.946582 \\
\hline 43 & 6 & 0 & -0.384007 & 4.866864 & -1.552560 \\
\hline 44 & 1 & 0 & -6.064874 & -0.338945 & 1.252325 \\
\hline 45 & 1 & 0 & 0.331185 & 5.619325 & -1.875416 \\
\hline 46 & 6 & 0 & -4.063996 & -1.683779 & 0.123867 \\
\hline 47 & 6 & 0 & -3.953421 & -2.557479 & 1.234043 \\
\hline 48 & 6 & 0 & -4.221158 & -2.221100 & -1.175837 \\
\hline 49 & 6 & 0 & -4.046591 & -3.938486 & 1.023296 \\
\hline 50 & 6 & 0 & -4.291367 & -3.611237 & -1.329575 \\
\hline 51 & 6 & 0 & -4.226887 & -4.491158 & -0.246288 \\
\hline 52 & 1 & 0 & -3.980301 & -4.598867 & 1.883538 \\
\hline 53 & 1 & 0 & -4.430861 & -4.023392 & -2.327351 \\
\hline 54 & 6 & 0 & 1.499134 & 3.747836 & -0.368578 \\
\hline 55 & 6 & 0 & 2.484960 & 3.479115 & -1.347832 \\
\hline 56 & 6 & 0 & 1.891579 & 4.030241 & 0.963131 \\
\hline 57 & 6 & 0 & 3.834266 & 3.493875 & -0.967527 \\
\hline 58 & 6 & 0 & 3.251954 & 4.033989 & 1.285648 \\
\hline 59 & 6 & 0 & 4.244616 & 3.772454 & 0.336810 \\
\hline 60 & 1 & 0 & 4.593597 & 3.295962 & -1.721677 \\
\hline 61 & 1 & 0 & 3.543347 & 4.268329 & 2.306626 \\
\hline 62 & 6 & 0 & 5.726187 & 3.843157 & 0.687445 \\
\hline 63 & 1 & 0 & 6.282028 & 3.479159 & -0.188116 \\
\hline 64 & 6 & 0 & 6.103544 & 2.945265 & 1.879378 \\
\hline 65 & 1 & 0 & 5.816859 & 1.902647 & 1.700949 \\
\hline 66 & 1 & 0 & 7.185016 & 2.976826 & 2.059115 \\
\hline 67 & 1 & 0 & 5.606709 & 3.269607 & 2.801043 \\
\hline 68 & 6 & 0 & 6.169617 & 5.297976 & 0.939736 \\
\hline 69 & 1 & 0 & 5.651540 & 5.721716 & 1.808336 \\
\hline 70 & 1 & 0 & 7.248204 & 5.349755 & 1.133328 \\
\hline 71 & 1 & 0 & 5.945871 & 5.933565 & 0.076187 \\
\hline 72 & 6 & 0 & -4.415856 & -5.988730 & -0.460318 \\
\hline 73 & 1 & 0 & -4.251226 & -6.182712 & -1.52985 \\
\hline 74 & 6 & 0 & -3.416719 & -6.855904 & 0.324431 \\
\hline 75 & 1 & 0 & -3.572613 & -6.773115 & 1.406400 \\
\hline 76 & 1 & 0 & -3.539868 & -7.912754 & 0.058844 \\
\hline 77 & 1 & 0 & -2.381602 & -6.567305 & 0.114221 \\
\hline 78 & 6 & 0 & -5.866169 & -6.404131 & -0.138313 \\
\hline 79 & 1 & 0 & -6.023391 & -7.469589 & -0.347411 \\
\hline 80 & 1 & 0 & -6.094485 & -6.230174 & 0.920167 \\
\hline 81 & 1 & 0 & -6.584157 & -5.828740 & -0.732761 \\
\hline 82 & 6 & 0 & 0.884636 & 4.395487 & 2.053633 \\
\hline
\end{tabular}

\begin{tabular}{|c|c|c|}
\hline & & \\
\hline 176148 & 685296 & $.985 / 81$ \\
\hline 768065 & .397220 & \\
\hline 916700 & 461364 & \\
\hline 065412 & 856438 & .512070 \\
\hline 977072 & 550184 & \\
\hline 301690 & & \\
\hline & & \\
\hline & & \\
\hline & & \\
\hline & & \\
\hline & & \\
\hline 2.362 & & \\
\hline .718522 & 705 & \\
\hline 698886 & & \\
\hline 4640 & & \\
\hline 080 & 257 & \\
\hline 662 & & \\
\hline 507 & & \\
\hline-3.5 & & \\
\hline-4.9 & & \\
\hline-5.1 & & \\
\hline-4.8 & & \\
\hline & & \\
\hline & & \\
\hline-1.5 & -2 & \\
\hline-2.3 & & \\
\hline-2.5 & -3 & \\
\hline$-4 \cdot 3$ & -1 & \\
\hline-4.3 & -0 . & \\
\hline-3.2 & -1 & \\
\hline-2.2 & -1 & \\
\hline-3.2 & & \\
\hline-3.3 & & \\
\hline-5.7 & & \\
\hline-6.5 & & \\
\hline-5.8 & & \\
\hline-5. & & \\
\hline & -3.8 & \\
\hline 0.2 & -3.53 & \\
\hline 2.0 & 5.1 & \\
\hline-0.3 & -4.3 & -0 \\
\hline-0.2 & -2 . & \\
\hline 1.4 & 5. & \\
\hline 2.9 & & \\
\hline 0.2 & & \\
\hline-1.3 & & \\
\hline & & \\
\hline-0.2 & & \\
\hline & & \\
\hline & & \\
\hline 0.92 & -2.2 & -2 . \\
\hline 5.32 & 2 . & \\
\hline 1.9 & -2. & \\
\hline 3.1 & -3.0 & \\
\hline & 3. & \\
\hline & & \\
\hline$-5.4 C$ & & \\
\hline-4 & & \\
\hline-2 . & & \\
\hline-1. & & \\
\hline & & \\
\hline & & \\
\hline 4.07 & 4 & \\
\hline 2.53 & & \\
\hline 4.01 & 0 . & \\
\hline 4.29 & -2.1 & 54 \\
\hline 5.20 & -1 & -2 \\
\hline-6.5 & & \\
\hline-4.5 & & \\
\hline-6. & & \\
\hline-7 . & & \\
\hline 5 . & & \\
\hline & & \\
\hline-4.0 & & \\
\hline-4.36 & & \\
\hline-5.37 & & -3.3 \\
\hline-2.12 & & -2.8 \\
\hline-3.41 & 06 & -3.35 \\
\hline-3.712 & .851825 & -4.050 \\
\hline-7 . & .521688 & .24 \\
\hline & 6836 & .81 \\
\hline & .366 & \\
\hline & & \\
\hline
\end{tabular}

\section{TSminor}

E (RB3LYP) $=-3736.08455715 \mathrm{~A} . \mathrm{U}$.

Sum of electronic and thermal Free Energies $=-3734.791499$ A.U. 


\begin{tabular}{|c|c|c|c|c|c|}
\hline \multirow{2}{*}{$\begin{array}{l}\text { Center } \\
\text { Number }\end{array}$} & & Atomic & & rdinates (A & Angstroms) \\
\hline & Number & Type & $\mathrm{x}$ & $\mathrm{Y}$ & z \\
\hline 1 & 8 & 0 & -0.397536 & -0.592826 & 1.166685 \\
\hline 2 & 15 & 0 & -0.318444 & 0.316445 & -0.038130 \\
\hline 3 & 8 & 0 & 0.209391 & 1.784094 & 0.531826 \\
\hline 4 & 8 & 0 & -1.855331 & 0.685951 & -0.568520 \\
\hline 5 & 8 & 0 & 0.475371 & -0.121115 & -1.238561 \\
\hline 6 & 6 & 0 & -0.020290 & 2.942140 & -0.192524 \\
\hline 7 & 6 & 0 & -2.697741 & 1.418482 & 0.253005 \\
\hline 8 & 6 & 0 & -1.316393 & 3.431870 & -0.265400 \\
\hline 9 & 6 & 0 & 1.092721 & 3.646488 & -0.743129 \\
\hline 10 & 6 & 0 & -2.413973 & 2.759417 & 0.481974 \\
\hline 11 & 6 & 0 & -3.890172 & 0.805852 & 0.747028 \\
\hline 12 & 6 & 0 & 0.831361 & 4.810354 & -1.438543 \\
\hline 13 & 6 & 0 & -4.698997 & 1.555742 & 1.578420 \\
\hline 14 & 1 & 0 & 1.665868 & 5.376920 & -1.843500 \\
\hline 15 & 1 & 0 & -5.630600 & 1.122499 & 1.932016 \\
\hline 16 & 6 & 0 & 2.522022 & 3.229839 & -0.556752 \\
\hline 17 & 6 & 0 & 3.265949 & 2.732091 & -1.654649 \\
\hline 18 & 6 & 0 & 3.166515 & 3.463914 & 0.678017 \\
\hline 19 & 6 & 0 & 4.633200 & 2.497429 & -1.486673 \\
\hline 20 & 6 & 0 & 4.541716 & 3.217666 & 0.787726 \\
\hline 21 & 6 & 0 & 5.301146 & 2.748195 & -0.283907 \\
\hline 22 & 1 & 0 & 5.207132 & 2.118746 & -2.330254 \\
\hline 23 & 1 & 0 & 5.026867 & 3.415490 & 1.738377 \\
\hline 24 & 6 & 0 & -4.396474 & -0.545843 & 0.325509 \\
\hline 25 & 6 & 0 & -4.364749 & -1.638279 & 1.223007 \\
\hline 26 & 6 & 0 & -5.079983 & -0.668980 & -0.909472 \\
\hline 27 & 6 & 0 & -5.053703 & -2.809797 & 0.882510 \\
\hline 28 & 6 & 0 & -5.745229 & -1.865666 & -1.201006 \\
\hline 29 & 6 & 0 & -5.764501 & -2.943547 & -0.310815 \\
\hline 30 & 1 & 0 & -5.048146 & -3.645218 & 1.578430 \\
\hline 31 & 1 & 0 & -6.285428 & -1.945237 & -2.141618 \\
\hline 32 & 6 & 0 & -6.565681 & -4.207816 & -0.598101 \\
\hline 33 & 1 & 0 & -6.337750 & -4.921172 & 0.205892 \\
\hline 34 & 6 & 0 & -6.174552 & -4.876685 & -1.927799 \\
\hline 35 & 1 & 0 & -5.105336 & -5.110969 & -1.953271 \\
\hline 36 & 1 & 0 & -6.733725 & -5.809759 & -2.067955 \\
\hline 37 & 1 & 0 & -6.398000 & -4.228079 & -2.783625 \\
\hline 38 & 6 & 0 & -8.081002 & -3.928955 & -0.549299 \\
\hline 39 & 1 & 0 & -8.378074 & -3.224627 & -1.335620 \\
\hline 40 & 1 & 0 & -8.652019 & -4.854207 & -0.694741 \\
\hline 41 & 1 & 0 & -8.372271 & -3.494873 & 0.413388 \\
\hline 42 & 6 & 0 & 6.813460 & 2.546772 & -0.216217 \\
\hline 43 & 1 & 0 & 7.025671 & 1.573311 & -0.682463 \\
\hline 44 & 6 & 0 & 7.540441 & 3.624059 & -1.049239 \\
\hline 45 & 1 & 0 & 7.364556 & 4.620865 & -0.626682 \\
\hline 46 & 1 & 0 & 8.622882 & 3.445327 & -1.057911 \\
\hline 47 & 1 & 0 & 7.189868 & 3.636829 & -2.086536 \\
\hline 48 & 6 & 0 & 7.386679 & 2.506596 & 1.206560 \\
\hline 49 & 1 & 0 & 8.454703 & 2.261430 & 1.174993 \\
\hline 50 & 1 & 0 & 7.291380 & 3.478149 & 1.706841 \\
\hline 51 & 1 & 0 & 6.882708 & 1.754539 & 1.821377 \\
\hline 52 & 6 & 0 & -5.147542 & 0.473864 & -1.923627 \\
\hline 53 & 1 & 0 & -4.640017 & 1.340543 & -1.492455 \\
\hline 54 & 6 & 0 & -4.401694 & 0.122627 & -3.225214 \\
\hline 55 & 1 & 0 & -4.434049 & 0.966543 & -3.924932 \\
\hline 56 & 1 & 0 & -3.350600 & -0.105706 & -3.021137 \\
\hline 57 & 1 & 0 & -4.853652 & -0.742144 & -3.727304 \\
\hline 58 & 6 & 0 & -6.598646 & 0.905443 & -2.210344 \\
\hline 59 & 1 & 0 & -7.117505 & 1.187697 & -1.287418 \\
\hline 60 & 1 & 0 & -6.612621 & 1.769150 & -2.885980 \\
\hline 61 & 1 & 0 & -7.176466 & 0.104446 & -2.686548 \\
\hline 62 & 6 & 0 & -3.629814 & -1.566389 & 2.562594 \\
\hline 63 & 1 & 0 & -2.927873 & -0.728479 & 2.510102 \\
\hline 64 & 6 & 0 & -2.798325 & -2.829649 & 2.850306 \\
\hline 65 & 1 & 0 & -2.148283 & -3.081892 & 2.008320 \\
\hline 66 & 1 & 0 & -2.164856 & -2.668857 & 3.729852 \\
\hline 67 & 1 & 0 & -3.431384 & -3.700334 & 3.060226 \\
\hline 68 & 6 & 0 & -4.601208 & -1.309646 & 3.732894 \\
\hline 69 & 1 & 0 & -5.341071 & -2.116092 & 3.811022 \\
\hline 70 & 1 & 0 & -4.053219 & -1.263348 & 4.682040 \\
\hline 71 & 1 & 0 & -5.143825 & -0.367306 & 3.613381 \\
\hline 72 & 6 & 0 & 2.639019 & 2.481594 & -3.027370 \\
\hline 73 & 1 & 0 & 1.557998 & 2.615550 & -2.934482 \\
\hline 74 & 6 & 0 & 3.147501 & 3.499345 & -4.068100 \\
\hline 75 & 1 & 0 & 4.229242 & 3.403541 & -4.222826 \\
\hline 76 & 1 & 0 & 2.655446 & 3.338694 & -5.035239 \\
\hline 77 & 1 & 0 & 2.948821 & 4.530391 & -3.755400 \\
\hline 78 & 6 & 0 & 2.861311 & 1.041651 & -3.522101 \\
\hline 79 & 1 & 0 & 2.422129 & 0.331251 & -2.818949 \\
\hline 80 & 1 & 0 & 2.375978 & 0.901212 & -4.496056 \\
\hline 81 & 1 & 0 & 3.926070 & 0.808595 & -3.645871 \\
\hline 82 & 6 & 0 & 2.427447 & 4.029768 & 1.890821 \\
\hline 83 & 1 & 0 & 1.382952 & 4.188069 & 1.609343 \\
\hline 84 & 6 & 0 & 2.425824 & 3.045847 & 3.075517 \\
\hline 85 & 1 & 0 & 1.958319 & 2.099172 & 2.787981 \\
\hline 86 & 1 & 0 & 3.443783 & 2.837232 & 3.427110 \\
\hline 87 & 1 & 0 & 1.861092 & 3.462978 & 3.918409 \\
\hline 88 & 6 & 0 & 2.988456 & 5.403463 & 2.307887 \\
\hline
\end{tabular}

\begin{tabular}{|c|c|c|}
\hline 2.949916 & 6.115282 & 1.475641 \\
\hline 2.405258 & 5.819356 & 3.138628 \\
\hline 4.031800 & 5.332462 & 2.637548 \\
\hline-0.482555 & 5.287916 & -1.662889 \\
\hline-1.584128 & 4.587099 & -1.078545 \\
\hline-3.205275 & 3.491039 & 1.432949 \\
\hline-4.367763 & 2.870324 & 1.986696 \\
\hline-0.725279 & 6.441104 & -2.456969 \\
\hline-2.007476 & 6.877843 & -2.695469 \\
\hline-2.183084 & 7.756683 & -3.310011 \\
\hline-2.897621 & 5.056873 & -1.360221 \\
\hline-3.102334 & 6.167936 & -2.148422 \\
\hline-4.116274 & 6.499869 & -2.355638 \\
\hline 0.126862 & 6.965190 & -2.883691 \\
\hline-3.749130 & 4.521886 & -0.956251 \\
\hline-2.871771 & 4.800993 & 1.877640 \\
\hline-3.657324 & 5.460766 & 2.796907 \\
\hline-3.374602 & 6.456193 & 3.129037 \\
\hline-5.168974 & 3.583613 & 2.918430 \\
\hline-4.825206 & 4.854343 & 3.317246 \\
\hline-5.439607 & 5.389201 & 4.036468 \\
\hline-1.976148 & 5.276850 & 1.495218 \\
\hline-6.055434 & 3.097701 & 3.319728 \\
\hline 0.610927 & -4.072497 & 0.064064 \\
\hline 0.514460 & -3.243892 & 1.140459 \\
\hline 1.118540 & -3.450817 & 2.395920 \\
\hline 1.020684 & -2.412658 & 3.341307 \\
\hline 1.761104 & -4.647534 & 2.757443 \\
\hline 1.564386 & -2.573530 & 4.612393 \\
\hline 0.509938 & -1.496877 & 3.061409 \\
\hline 2.306544 & -4.788608 & 4.032869 \\
\hline 1.824517 & -5.483451 & 2.068808 \\
\hline 2.215360 & -3.756835 & 4.967645 \\
\hline 1.476334 & -1.762821 & 5.330542 \\
\hline 2.797444 & -5.721555 & 4.296687 \\
\hline 2.639492 & -3.876669 & 5.960211 \\
\hline 3.792131 & -1.457773 & 0.278381 \\
\hline 2.606813 & -2.942805 & -1.406900 \\
\hline 2.824376 & -1.765427 & -0.707813 \\
\hline 2.053842 & -1.083619 & -0.808153 \\
\hline 1.663705 & -3.577713 & -0.724708 \\
\hline 3.676146 & -4.015949 & -1.425494 \\
\hline 4.286639 & -4.393790 & -2.629935 \\
\hline 4.046031 & -4.688964 & -0.249534 \\
\hline 5.249805 & -5.405035 & -2.655980 \\
\hline 4.019135 & -3.894893 & -3.555116 \\
\hline 5.002367 & -5.701150 & -0.276183 \\
\hline 3.594798 & -4.407769 & 0.697145 \\
\hline 5.609625 & -6.063612 & -1.481121 \\
\hline 5.713987 & -5.677745 & -3.599825 \\
\hline 5.274879 & -6.206220 & 0.646470 \\
\hline 6.354500 & -6.854326 & -1.502599 \\
\hline 0.066774 & -2.311497 & 1.032738 \\
\hline-0.467808 & -4.176261 & -0.948598 \\
\hline-1.466547 & -3.206251 & -1.117839 \\
\hline-0.446565 & -5.297550 & -1.798298 \\
\hline-2.415814 & -3.356161 & -2.127855 \\
\hline-1.516408 & -2.339943 & -0.468347 \\
\hline-1.396205 & -5.442894 & -2.804427 \\
\hline 0.324535 & -6.054090 & -1.668305 \\
\hline-2.380424 & -4.465322 & -2.975404 \\
\hline-3.187428 & -2.603242 & -2.242164 \\
\hline-1.370765 & -6.315452 & -3.451348 \\
\hline-3.121331 & -4.570902 & -3.762925 \\
\hline 3.375839 & -0.759357 & 1.422095 \\
\hline 6.064002 & -1.435534 & 1.117757 \\
\hline 2.328285 & -0.499242 & 1.535228 \\
\hline 7.112925 & -1.687482 & 0.985638 \\
\hline 4.298712 & -0.421195 & 2.409623 \\
\hline 5.641803 & -0.774135 & 2.274034 \\
\hline 3.957908 & 0.110186 & 3.293118 \\
\hline 6.355952 & -0.527078 & 3.054631 \\
\hline 1.080068 & -5.029413 & 0.285032 \\
\hline 1.866470 & -2.698690 & -2.717937 \\
\hline 2.540582 & -2.216504 & -3.435099 \\
\hline 1.024900 & -2.021347 & -2.553099 \\
\hline 1.503440 & -3.635004 & -3.146597 \\
\hline 5.153427 & -1.765645 & 0.116972 \\
\hline 5.497097 & -2.248476 & -0.789462 \\
\hline
\end{tabular}


TS'major

E (RB3LYP) $=-3736.09042131 \mathrm{~A} . \mathrm{H}$.

Sum of electronic and thermal Free Energies $=-3734.800012 \mathrm{~A} \cdot \mathrm{U}$.

Center Atomic Atomic Coordinates (Angstroms)

Number Type

$\begin{array}{rrrrrc}1 & 6 & 0 & 2.039335 & -3.002081 & 1.756084 \\ 2 & 7 & 0 & 0.813453 & -2.981765 & 1.163803 \\ 3 & 6 & 0 & 0.327692 & -3.947147 & 0.262781 \\ 4 & 6 & 0 & -0.868732 & -3.658921 & -0.416922 \\ 5 & 6 & 0 & 0.962151 & -5.181134 & 0.038120 \\ 6 & 6 & 0 & -1.400943 & -4.579514 & -1.315054 \\ 7 & 1 & 0 & -1.375952 & -2.717915 & -0.231071 \\ 8 & 6 & 0 & 0.415876 & -6.093421 & -0.863234 \\ 9 & 1 & 0 & 1.867066 & -5.453217 & 0.571239 \\ 10 & 6 & 0 & -0.763016 & -5.800335 & -1.549126 \\ 11 & 1 & 0 & -2.325462 & -4.332759 & -1.827298\end{array}$

$0.919633-7.042673-1.024522$

$\begin{array}{lll}-1.180307 & -6.514276 & -2.253161\end{array}$

$\begin{array}{lll}2.762969 & -2.403100 & -2.380953\end{array}$

$\begin{array}{lll}3.576267 & -1.690179 & -0.067000\end{array}$

$2.768432-1.645363-1.189960$

$1.942421-1.026617-1.098962$

$\begin{array}{lll}2.900685 & -2.370782 & 0.851671\end{array}$

$\begin{array}{lll}4.852625 & -2.503646 & -0.081740\end{array}$

$\begin{array}{lll}6.100372 & -1.883875 & 0.071315\end{array}$

$4.810169-3.903309-0.195550$

$\begin{array}{lll}7.274284 & -2.640673 \quad 0.095286\end{array}$

$\begin{array}{lll}6.162985 & -0.804810 & 0.163467\end{array}$

$\begin{array}{llll}5.979697 & -4.658746 & -0.165767\end{array}$

$\begin{array}{lll}3.854374 & -4.399993 & -0.334685\end{array}$

$\begin{array}{lll}7.218645 & -4.028926 & -0.019897\end{array}$

$\begin{array}{lll}8.231738 & -2.139782 & 0.209172\end{array}$

$\begin{array}{lll}5.924519 & -5.739911 & -0.258846\end{array}$

$\begin{array}{lll}8.131587 & -4.617430 & 0.005310\end{array}$

$\begin{array}{lll}0.261514 & -2.100590 & 1.213154\end{array}$

$\begin{array}{lll}0.441875 & -0.034823 & -1.157597\end{array}$

$\begin{array}{lll}-0.496042 & 0.315406 & -0.036430\end{array}$

$\begin{array}{lll}-2.004585 & 0.460333 & -0.710160\end{array}$

$\begin{array}{lll}-0.232301 & 1.859997 & 0.514614\end{array}$

$\begin{array}{lll}-0.551968 & -0.549691 & 1.200357\end{array}$

$\begin{array}{lll}-3.010078 & 1.113187 & -0.018142\end{array}$

$\begin{array}{lll}-0.522164 & 2.951723 & -0.283822\end{array}$

$\begin{array}{lll}-2.944929 & 2.494437 & 0.116766\end{array}$

$\begin{array}{lll}-4.125790 & 0.350267 & 0.441403\end{array}$

$\begin{array}{lll}-1.851351 & 3.266108 & -0.537837\end{array}$

$\begin{array}{llll}0.557138 & 3.777173 & -0.723374\end{array}$

$\begin{array}{lll}-5.135153 & 1.026744 & 1.097247\end{array}$

$\begin{array}{lll}0.245489 & 4.883520 & -1.488315\end{array}$

$\begin{array}{lll}-6.009904 & 0.472850 & 1.427621\end{array}$

$\begin{array}{lll}1.047622 & 5.542316 & -1.811196\end{array}$

$\begin{array}{lll}-4.284507 & -1.125154 & 0.214489\end{array}$

$\begin{array}{lll}-4.261697 & -2.014809 & 1.317733\end{array}$

$\begin{array}{lll}-4.599921 & -1.616712 & -1.074317\end{array}$

$\begin{array}{lll}-4.596798 & -3.358509 & 1.112902\end{array}$

$\begin{array}{lll}-4.914867 & -2.973215 & -1.222341\end{array}$

$\begin{array}{lll}-4.940701 & -3.860794 & -0.143875\end{array}$

$\begin{array}{lll}-4.594767 & -4.028743 & 1.968156\end{array}$

$\begin{array}{lll}-5.178790 & -3.346555 & -2.210258\end{array}$

$\begin{array}{lll}1.991860 & 3.523552 & -0.364559\end{array}$

$\begin{array}{lll}2.925117 & 3.188144 & -1.375139\end{array}$

$\begin{array}{lll}2.441224 & 3.734897 & 0.962560\end{array}$

$\begin{array}{lll}4.280957 & 3.086406 & -1.033792\end{array}$

$\begin{array}{lll}3.806009 & 3.619117 & 1.245952\end{array}$

$\begin{array}{lll}4.749014 & 3.306769 & 0.262649\end{array}$

$\begin{array}{lll}5.000570 & 2.842436 & -1.813038\end{array}$

$\begin{array}{lll}4.142290 & 3.803926 & 2.263176\end{array}$

$\begin{array}{lll}6.242666 & 3.267968 & 0.564800\end{array}$

$\begin{array}{lll}6.738618 & 2.841403 & -0.318568\end{array}$

$\begin{array}{lll}6.595800 & 2.375734 & 1.768023\end{array}$

$\begin{array}{lll}6.224733 & 1.353521 & 1.633300\end{array}$

$\begin{array}{lll}7.682761 & 2.328725 & 1.905716\end{array}$

$\begin{array}{lll}6.164043 & 2.763212 & 2.698063\end{array}$

$\begin{array}{lll}6.802387 & 4.691318 & 0.761307\end{array}$

$\begin{array}{lll}6.346667 & 5.174511 & 1.633836\end{array}$

$\begin{array}{lll}7.887816 & 4.666346 & 0.918501\end{array}$

$\begin{array}{lll}6.597207 & 5.319651 & -0.112061\end{array}$

$\begin{array}{lll}-5.394343 & -5.302481 & -0.343654\end{array}$

$\begin{array}{lll}-5.342389 & -5.508239 & -1.422329\end{array}$

$\begin{array}{lll}-4.500947 & -6.334606 & 0.365521\end{array}$

$\begin{array}{lll}-4.555670 & -6.234531 & 1.455906\end{array}$

$\begin{array}{lll}-4.825237 & -7.352176 & 0.116317\end{array}$

$\begin{array}{lll}-3.451945 & -6.226786 & 0.071702\end{array}$

$\begin{array}{lll}-6.866988 & -5.471422 & 0.083445\end{array}$

$\begin{array}{lll}-7.216425 & -6.492344 & -0.114527\end{array}$

$\begin{array}{lll}-6.986030 & -5.275869 & 1.156023\end{array}$

$\begin{array}{lll}-7.518592 & -4.775558 & -0.45625\end{array}$

$\begin{array}{lll}1.498349 & 4.148377 & 2.092730\end{array}$

$\begin{array}{lll}0.482352 & 4.189777 & 1.693095\end{array}$

$\begin{array}{lll}1.487211 & 3.121134 & 3.239809 \\ 0.772645 & 3.425825 & 4.014448\end{array}$

\begin{tabular}{|c|c|c|}
\hline .190742 & .134845 & \\
\hline .472532 & 3.031455 & .713505 \\
\hline 1.830229 & .561729 & .611573 \\
\hline 1.797281 & .298688 & .801261 \\
\hline 1.107833 & .866938 & .378365 \\
\hline .829737 & .604271 & 3.060684 \\
\hline .525409 & .948303 & \\
\hline .439912 & 046480 & \\
\hline .869582 & 3093 & \\
\hline .332674 & & \\
\hline & & \\
\hline & & \\
\hline 3.1471 & & \\
\hline 4.2 & & \\
\hline 2.80 & & \\
\hline 2.875274 & & \\
\hline-3.895519 & & \\
\hline-3.572311 & 22984 & \\
\hline-5.107864 & 281 & \\
\hline-5.471239 & & \\
\hline-4.834745 & & \\
\hline-5.943 & & \\
\hline-2 . & & \\
\hline-1.8. & & \\
\hline- & & \\
\hline-2 . & & \\
\hline-4.66 & & \\
\hline-4.410 & & \\
\hline-3.626 & -1 & 426 \\
\hline-2.613 & -1 & \\
\hline-3.818 & & \\
\hline-3.66 & & \\
\hline-6.08 & & -2 \\
\hline-6.81 & & \\
\hline-6 . & & \\
\hline-6.4 & & \\
\hline 2.3 & & \\
\hline & & \\
\hline & & \\
\hline & & \\
\hline 0.636 & & \\
\hline 3.762428 & -2 . & 309 \\
\hline 4.070 & -3 & \\
\hline 2.95 & & \\
\hline 1.20 & & \\
\hline 4.63 & & \\
\hline .20 & & \\
\hline .51 & & \\
\hline 3 & & \\
\hline & & \\
\hline & & \\
\hline & & \\
\hline 2.586941 & -3. & -4 \\
\hline 0.457211 & -3.48 & -4 . \\
\hline 2.5215 & -4 . & -5 \\
\hline$-5.0636^{\circ}$ & & \\
\hline-3.94 & & \\
\hline-2.1 & & -1 \\
\hline-1.07 & & \\
\hline 2.538 & -3.96 & \\
\hline 3.633 & & \\
\hline & & \\
\hline 2 & & \\
\hline 4 & & \\
\hline & & \\
\hline & -2 . & -2. \\
\hline-6.0722 & & \\
\hline-5.9713 & & \\
\hline-6.743914 & 2 & 3.0 \\
\hline-3.862003 & & 89 \\
\hline-4.845 & & \\
\hline-4.752 & & \\
\hline-6.92 & & \\
\hline-3.0 & & \\
\hline-3.45 & & \\
\hline 3.6 & & \\
\hline & & \\
\hline-1.356 & & \\
\hline-2.638 & & \\
\hline-2.84 & & \\
\hline-4.27 & & \\
\hline 0.529356 & 6.888322 & -3.10198 \\
\hline
\end{tabular}


TS'minor

Done: $E($ RB3LYP $)=-3736.08019477 \mathrm{~A}$.U.

Sum of electronic and thermal Free Energies $=-3734.789389$ A.U.

Center Atomic Atomic Coordinates (Angstroms)

Number Type

\begin{tabular}{|c|c|c|c|c|c|}
\hline 1 & 8 & 0 & 0.269693 & -0.814855 & -0.889306 \\
\hline 2 & 15 & 0 & 0.524467 & 0.228320 & 0.165332 \\
\hline 3 & 8 & 0 & 0.326385 & 1.679019 & -0.611028 \\
\hline 4 & 8 & 0 & 2.110772 & 0.313911 & 0.630250 \\
\hline 5 & 8 & 0 & -0.240051 & 0.148389 & 1.465222 \\
\hline 6 & 6 & 0 & 0.711678 & 2.875389 & -0.044749 \\
\hline 7 & 6 & 0 & 3.111869 & 0.866757 & -0.138611 \\
\hline 8 & 6 & 0 & 2.061567 & 3.131850 & 0.162980 \\
\hline 9 & 6 & 0 & -0.300846 & 3.861779 & 0.170476 \\
\hline 10 & 6 & 0 & 3.108008 & 2.230747 & -0.401581 \\
\hline 11 & 6 & 0 & 4.190245 & 0.011552 & -0.520208 \\
\hline 12 & 6 & 0 & 0.087387 & 5.056097 & 0.74330 \\
\hline 13 & 6 & 0 & 5.229764 & 0.567731 & -1.235728 \\
\hline 14 & 1 & 0 & -0.652669 & 5.838685 & 0.887534 \\
\hline 15 & 1 & 0 & 6.073974 & -0.058630 & -1.513685 \\
\hline 16 & 6 & 0 & -1.722638 & 3.670844 & -0.27208 \\
\hline 17 & 6 & 0 & -2.779841 & 3.705440 & 0.673454 \\
\hline 18 & 6 & 0 & -2.027515 & 3.554917 & -1.651491 \\
\hline 19 & 6 & 0 & -4.102216 & 3.630161 & 0.219831 \\
\hline 20 & 6 & 0 & -3.368337 & 3.474169 & -2.04518 \\
\hline 21 & 6 & 0 & -4.424326 & 3.519182 & -1.1348 \\
\hline 22 & 1 & 0 & -4.904946 & 3.671840 & 0.95225 \\
\hline 23 & 1 & 0 & -3.598934 & 3.397048 & -3.105502 \\
\hline 24 & 6 & 0 & 4.237438 & -1.442253 & -0.154687 \\
\hline 25 & 6 & 0 & 4.139968 & -2.426377 & -1.167013 \\
\hline 26 & 6 & 0 & 4.466805 & -1.835504 & 1.186823 \\
\hline 27 & 6 & 0 & 4.283648 & -3.775165 & -0.812542 \\
\hline 28 & 6 & 0 & 4.600540 & -3.195297 & 1.4828 \\
\hline 29 & 6 & 0 & 4.519530 & -4.185856 & 0.500336 \\
\hline 30 & 1 & 0 & 4.220244 & -4.532746 & -1.591700 \\
\hline 31 & 1 & 0 & 4.789889 & -3.486603 & 2.513113 \\
\hline 32 & 6 & 0 & 4.719594 & -5.659433 & 0.832697 \\
\hline 33 & 1 & 0 & 4.504569 & -6.227997 & -0.083171 \\
\hline 34 & 6 & 0 & 3.753675 & -6.158525 & 1.922850 \\
\hline 35 & 1 & 0 & 2.710063 & -5.979372 & 1.643353 \\
\hline 36 & 1 & 0 & 3.884613 & -7.234786 & 2.088974 \\
\hline 37 & 1 & 0 & 3.933919 & -5.653682 & 2.879162 \\
\hline 38 & 6 & 0 & 6.182712 & -5.951187 & 1.220249 \\
\hline 39 & 1 & 0 & 6.461988 & -5.414967 & 2.135076 \\
\hline 40 & 1 & 0 & 6.331430 & -7.022877 & 1.401099 \\
\hline 41 & 1 & 0 & 6.871690 & -5.638812 & 0.427891 \\
\hline 42 & 6 & 0 & -5.869561 & 3.488851 & -1.616902 \\
\hline 43 & 1 & 0 & -5.841580 & 3.390240 & -2.71074 \\
\hline 44 & 6 & 0 & -6.647932 & 2.278758 & -1.068488 \\
\hline 45 & 1 & 0 & -6.709655 & 2.307545 & 0.02625 \\
\hline 46 & 1 & 0 & -7.672931 & 2.266994 & -1.458825 \\
\hline 47 & 1 & 0 & -6.166467 & 1.336963 & -1.355390 \\
\hline 48 & 6 & 0 & -6.603182 & 4.804625 & -1.292833 \\
\hline 49 & 1 & 0 & -7.620337 & 4.793969 & -1.703246 \\
\hline 50 & 1 & 0 & -6.681528 & 4.959655 & -0.210143 \\
\hline 51 & 1 & 0 & -6.073250 & 5.665409 & -1.714649 \\
\hline 52 & 6 & 0 & 4.622982 & -0.825446 & 2.323569 \\
\hline 53 & 1 & 0 & 4.493790 & 0.177703 & 1.909492 \\
\hline 54 & 6 & 0 & 3.545053 & -1.007228 & 3.408849 \\
\hline 55 & 1 & 0 & 3.662764 & -0.247193 & 4.190976 \\
\hline 56 & 1 & 0 & 2.543431 & -0.902678 & 2.982142 \\
\hline 57 & 1 & 0 & 3.617315 & -1.990459 & 3.88981 \\
\hline 58 & 6 & 0 & 6.039575 & -0.877661 & 2.929782 \\
\hline 59 & 1 & 0 & 6.805186 & -0.707744 & 2.164304 \\
\hline 60 & 1 & 0 & 6.153497 & -0.107015 & 3.701859 \\
\hline 61 & 1 & 0 & 6.244705 & -1.848377 & 3.396731 \\
\hline 62 & 6 & 0 & 3.870171 & -2.084465 & -2.633586 \\
\hline 63 & 1 & 0 & 3.838783 & -0.996715 & -2.727372 \\
\hline 64 & 6 & 0 & 2.492954 & -2.601622 & -3.090272 \\
\hline 65 & 1 & 0 & 1.700956 & -2.165439 & -2.473782 \\
\hline 66 & 1 & 0 & 2.309210 & -2.318954 & -4.134493 \\
\hline 67 & 1 & 0 & 2.431185 & -3.695849 & -3.026750 \\
\hline 68 & 6 & 0 & 4.993226 & -2.586320 & -3.561073 \\
\hline 69 & 1 & 0 & 5.065829 & -3.680528 & -3.559480 \\
\hline 70 & 1 & 0 & 4.803600 & -2.269649 & -4.593751 \\
\hline 71 & 1 & 0 & 5.969649 & -2.189958 & -3.259093 \\
\hline 72 & 6 & 0 & -2.548438 & 3.833438 & 2.180478 \\
\hline 73 & 1 & 0 & -1.471499 & 3.805141 & 2.36352 \\
\hline 74 & 6 & 0 & -3.075730 & 5.176615 & 2.723939 \\
\hline 75 & 1 & 0 & -4.162490 & 5.258798 & 2.601547 \\
\hline 76 & 1 & 0 & -2.853107 & 5.271590 & 3.793766 \\
\hline 77 & 1 & 0 & -2.622075 & 6.029592 & 2.206963 \\
\hline 78 & 6 & 0 & -3.161287 & 2.656970 & 2.959951 \\
\hline 79 & 1 & 0 & -2.738500 & 1.706352 & 2.624572 \\
\hline 80 & 1 & 0 & -2.949801 & 2.758123 & 4.031013 \\
\hline 81 & 1 & 0 & -4.250873 & 2.610136 & 2.843970 \\
\hline 82 & 6 & 0 & -0.963972 & 3.583495 & -2.750044 \\
\hline 83 & 1 & 0 & 0.019625 & 3.642754 & -2.279712 \\
\hline 84 & 6 & 0 & -0.977709 & 2.303642 & -3.60710 \\
\hline 85 & 1 & 0 & -0.820211 & 1.415929 & -2.9884 \\
\hline
\end{tabular}

\begin{tabular}{|c|c|c|c|c|c|}
\hline 86 & 1 & 0 & -1.925900 & 2.187496 & -4.146229 \\
\hline 87 & 1 & 0 & -0.176595 & 2.342750 & -4.355183 \\
\hline 88 & 6 & 0 & -1.108490 & 4.843544 & -3.627942 \\
\hline 89 & 1 & 0 & -1.059339 & 5.756558 & -3.023592 \\
\hline 90 & 1 & 0 & -0.303193 & 4.883811 & -4.371433 \\
\hline 91 & 1 & 0 & -2.061814 & 4.852943 & -4.169656 \\
\hline 92 & 6 & 0 & 1.416888 & 5.295863 & 1.171157 \\
\hline 93 & 6 & 0 & 2.425527 & 4.321433 & 0.887091 \\
\hline 94 & 6 & 0 & 4.132981 & 2.764762 & -1.264129 \\
\hline 95 & 6 & 0 & 5.218116 & 1.919011 & -1.659622 \\
\hline 96 & 6 & 0 & 1.766754 & 6.484015 & 1.867590 \\
\hline 97 & 6 & 0 & 3.743277 & 4.567327 & 1.362665 \\
\hline 98 & 6 & 0 & 3.054712 & 6.696231 & 2.301946 \\
\hline 99 & 1 & 0 & 3.309411 & 7.604128 & 2.842025 \\
\hline 100 & 6 & 0 & 4.047198 & 5.718670 & 2.055659 \\
\hline 101 & 1 & 0 & 5.059049 & 5.875231 & 2.420000 \\
\hline 102 & 6 & 0 & 4.109279 & 4.092115 & -1.777336 \\
\hline 103 & 6 & 0 & 5.114208 & 4.560599 & -2.595192 \\
\hline 104 & 1 & 0 & 5.062252 & 5.575519 & -2.980367 \\
\hline 105 & 6 & 0 & 6.251168 & 2.437975 & -2.485907 \\
\hline 106 & 6 & 0 & 6.207461 & 3.733975 & -2.943908 \\
\hline 107 & 1 & 0 & 6.998159 & 4.118708 & -3.582316 \\
\hline 108 & 1 & 0 & 0.988579 & 7.218215 & 2.063493 \\
\hline 109 & 1 & 0 & 4.513637 & 3.824810 & 1.188577 \\
\hline 110 & 1 & 0 & 3.276859 & 4.740296 & -1.531739 \\
\hline 111 & 1 & 0 & 7.071908 & 1.780057 & -2.762457 \\
\hline 112 & 6 & 0 & -2.188014 & -3.032150 & 1.813377 \\
\hline 113 & 7 & 0 & -1.626859 & -2.043274 & 2.563149 \\
\hline 114 & 6 & 0 & -2.144411 & -1.539670 & 3.773966 \\
\hline 115 & 6 & 0 & -1.545496 & -0.381806 & 4.301837 \\
\hline 116 & 6 & 0 & -3.170141 & -2.173889 & 4.494958 \\
\hline 117 & 6 & 0 & -1.968440 & 0.122275 & 5.528192 \\
\hline 118 & 1 & 0 & -0.765657 & 0.112522 & 3.731258 \\
\hline 119 & 6 & 0 & -3.590210 & -1.647671 & 5.715218 \\
\hline 120 & 1 & 0 & -3.633578 & -3.085850 & 4.133443 \\
\hline 121 & 6 & 0 & -2.993900 & -0.501335 & 6.242510 \\
\hline 122 & 1 & 0 & -1.491825 & 1.014607 & 5.924449 \\
\hline 123 & 1 & 0 & -4.383559 & -2.150449 & 6.261767 \\
\hline 124 & 1 & 0 & -3.320931 & -0.103176 & 7.198557 \\
\hline 125 & 6 & 0 & -2.590059 & -2.296194 & -2.582189 \\
\hline 126 & 6 & 0 & -3.407938 & -1.692487 & -0.245058 \\
\hline 127 & 7 & 0 & -2.491053 & -1.727067 & -1.292631 \\
\hline 128 & 1 & 0 & -1.564875 & -1.315396 & -1.093189 \\
\hline 129 & 1 & 0 & -2.882826 & -2.404426 & 0.718804 \\
\hline 130 & 6 & 0 & -4.724640 & -2.415911 & -0.416753 \\
\hline 131 & 6 & 0 & -5.944406 & -1.772201 & -0.164823 \\
\hline 132 & 6 & 0 & -4.745102 & -3.777248 & -0.767870 \\
\hline 133 & 6 & 0 & -7.152662 & -2.464353 & -0.277413 \\
\hline 134 & 1 & 0 & -5.959589 & -0.724034 & 0.112537 \\
\hline 135 & 6 & 0 & -5.949258 & -4.468478 & -0.870468 \\
\hline 136 & 1 & 0 & -3.811694 & -4.292644 & -0.967973 \\
\hline 137 & 6 & 0 & -7.159989 & -3.813477 & -0.628086 \\
\hline 138 & 1 & 0 & -8.087147 & -1.943778 & -0.086779 \\
\hline 139 & 1 & 0 & -5.943388 & -5.519896 & -1.144893 \\
\hline 140 & 1 & 0 & -8.099775 & -4.352426 & -0.711688 \\
\hline 141 & 1 & 0 & -0.894140 & -1.446451 & 2.139319 \\
\hline 142 & 6 & 0 & -1.376832 & -4.004141 & 1.045434 \\
\hline 143 & 6 & 0 & -0.112680 & -3.708348 & 0.513594 \\
\hline 144 & 6 & 0 & -1.919600 & -5.288824 & 0.851671 \\
\hline 145 & 6 & 0 & 0.593531 & -4.685681 & -0.186137 \\
\hline 146 & 1 & 0 & 0.316169 & -2.718316 & 0.609355 \\
\hline 147 & 6 & 0 & -1.210338 & -6.261358 & 0.153367 \\
\hline 148 & 1 & 0 & -2.896994 & -5.525756 & 1.265217 \\
\hline 149 & 6 & 0 & 0.050374 & -5.959842 & -0.368118 \\
\hline 150 & 1 & 0 & 1.572266 & -4.442163 & -0.586222 \\
\hline 151 & 1 & 0 & -1.635747 & -7.252475 & 0.022864 \\
\hline 152 & 1 & 0 & 0.607984 & -6.716107 & -0.913853 \\
\hline 153 & 6 & 0 & -1.432996 & -2.861601 & -3.142843 \\
\hline 154 & 6 & 0 & -3.775853 & -2.746739 & -4.647414 \\
\hline 155 & 1 & 0 & -0.524464 & -2.894857 & -2.552394 \\
\hline 156 & 1 & 0 & -4.689226 & -2.684604 & -5.233269 \\
\hline 157 & 6 & 0 & -1.455659 & -3.364414 & -4.441391 \\
\hline 158 & 6 & 0 & -2.628202 & -3.320837 & -5.198307 \\
\hline 159 & 1 & 0 & -0.550005 & -3.795291 & -4.859762 \\
\hline 160 & 1 & 0 & -2.645096 & -3.717701 & -6.209490 \\
\hline 161 & 1 & 0 & -3.065121 & -3.481677 & 2.274058 \\
\hline 162 & 6 & 0 & -3.442951 & -0.346968 & 0.471659 \\
\hline 163 & 1 & 0 & -3.887966 & 0.411772 & -0.182065 \\
\hline 164 & 1 & 0 & -2.431307 & -0.015673 & 0.722122 \\
\hline 165 & 1 & 0 & -4.022635 & -0.407300 & 1.396864 \\
\hline 166 & 6 & 0 & -3.761502 & -2.225838 & -3.355187 \\
\hline 167 & 1 & 0 & -4.648323 & -1.748533 & -2.955656 \\
\hline
\end{tabular}




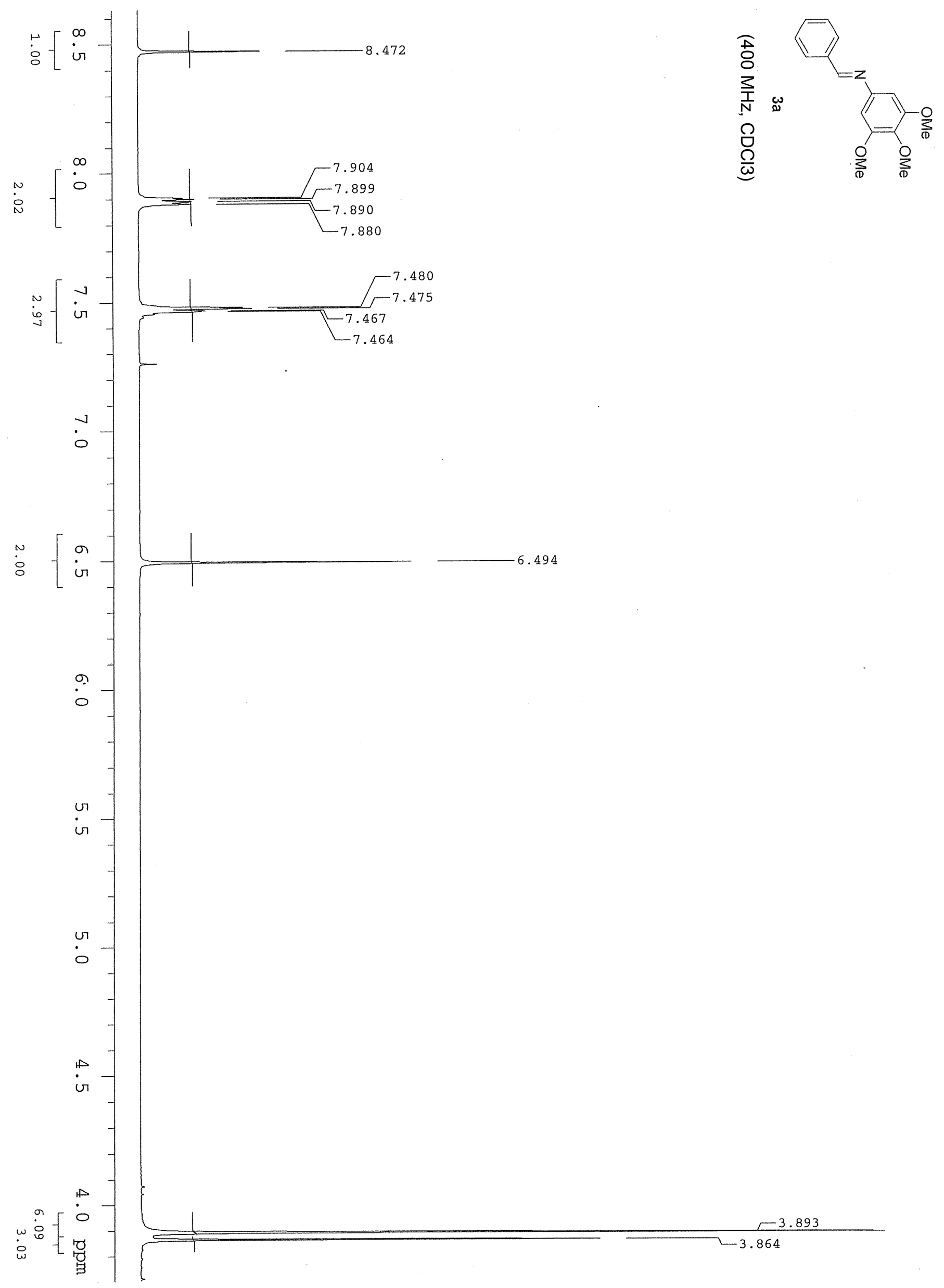




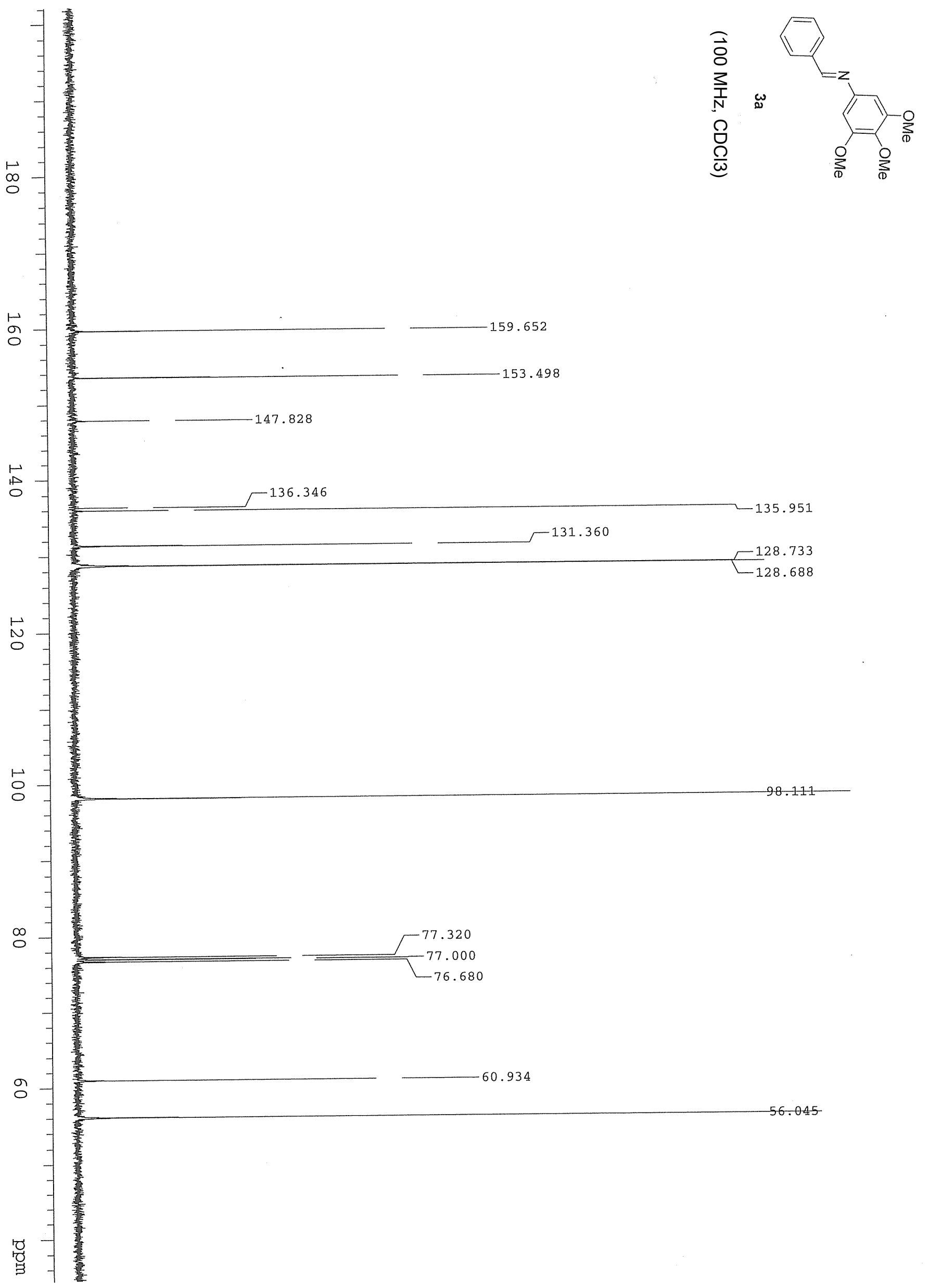




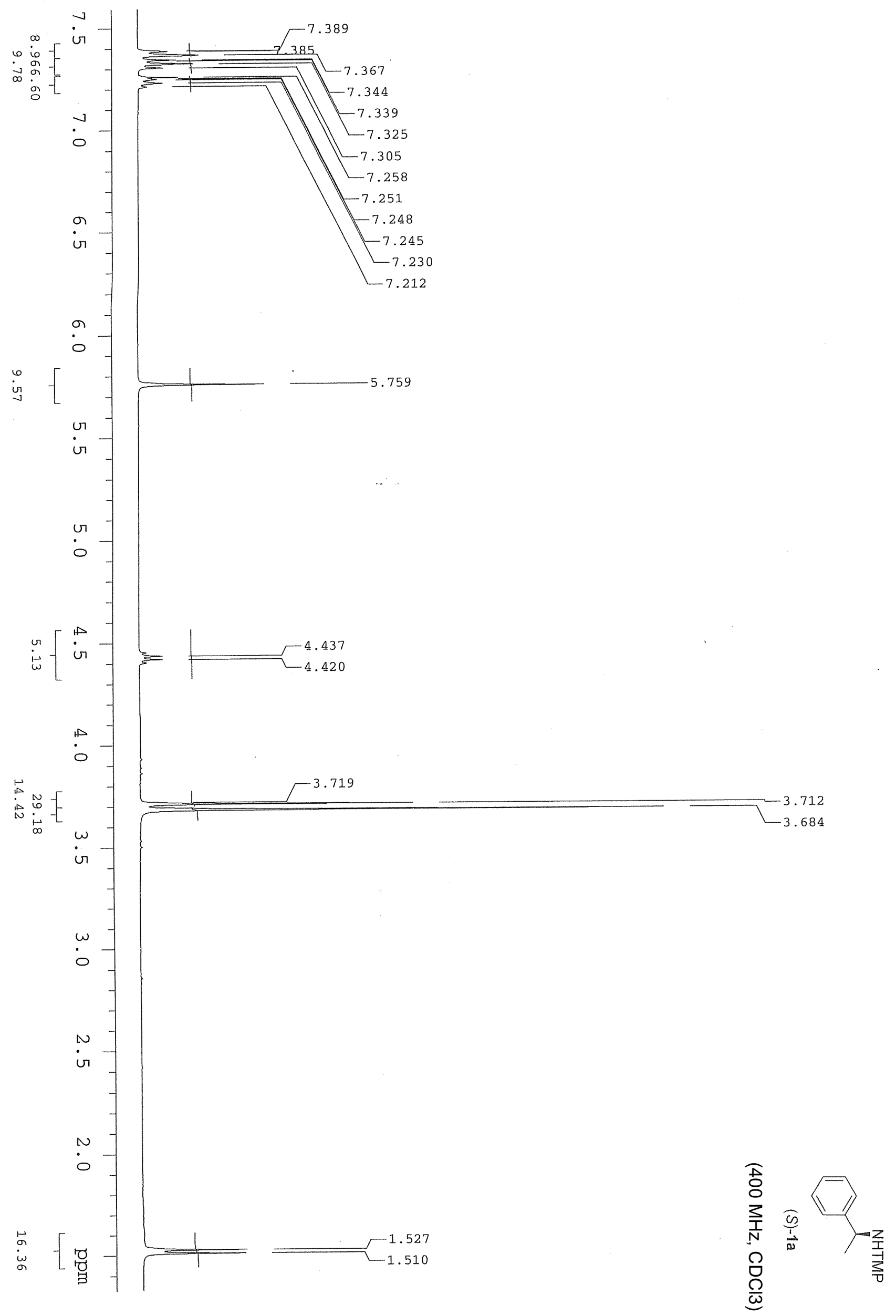



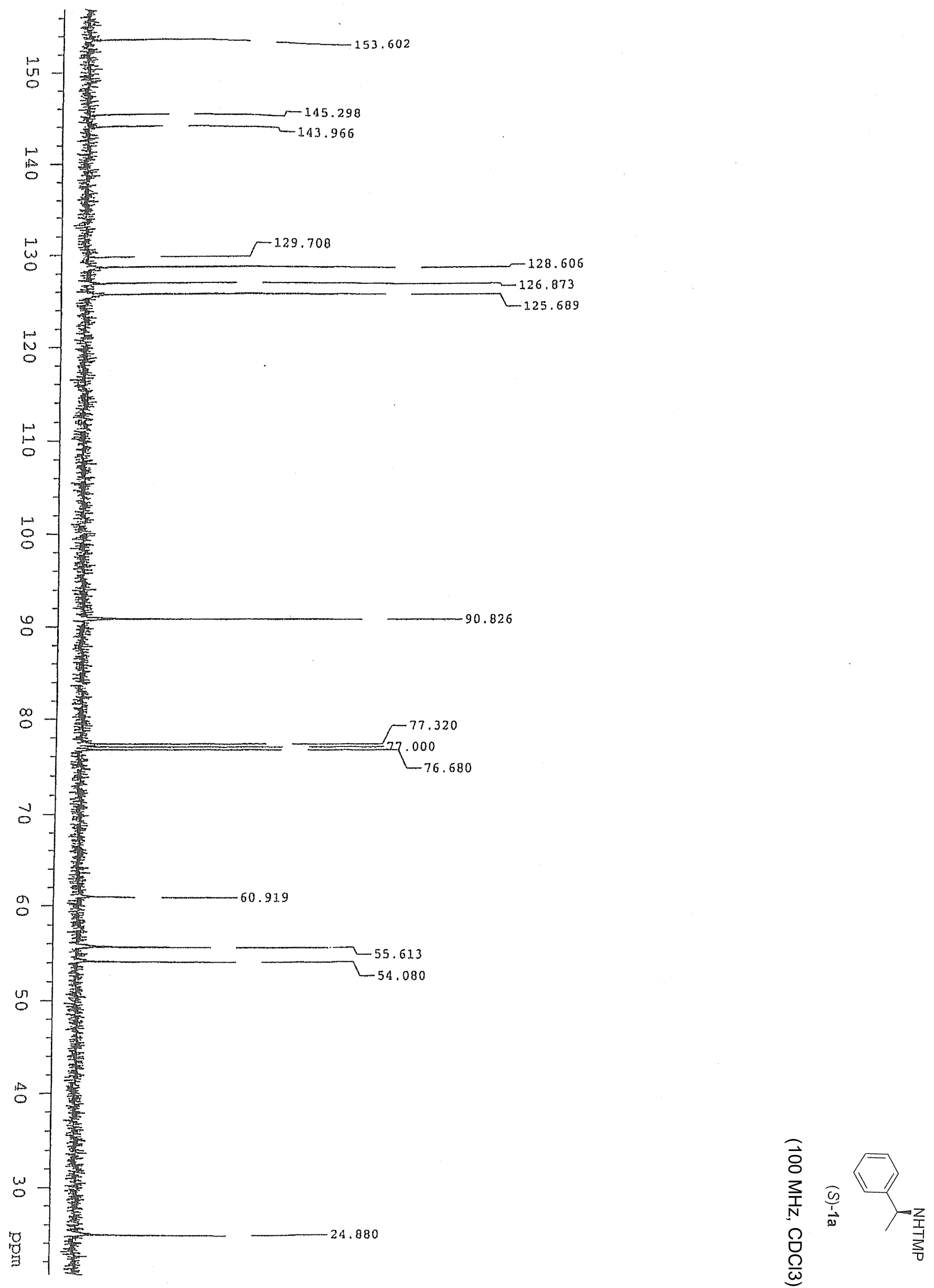
0.05

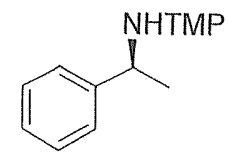

(S)-1a

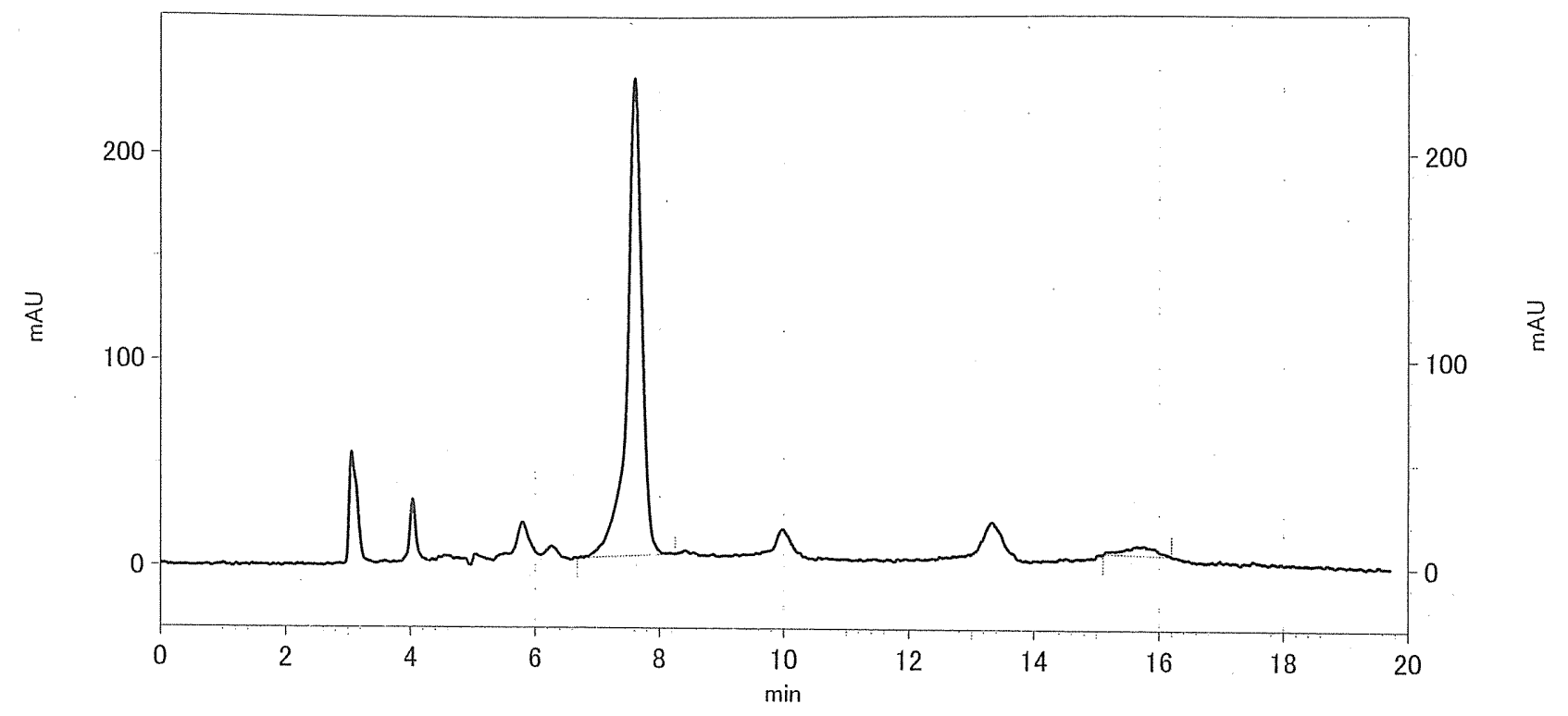

\begin{tabular}{ccccc} 
Peak \# & Retention time & Type & Area & Area \% \\
\hline 1 & 7.61 & IB & 15367748 & 95.602 \\
2 & 15.64 & $\mathrm{BI}$ & 706941 & 4.398
\end{tabular}

0.05
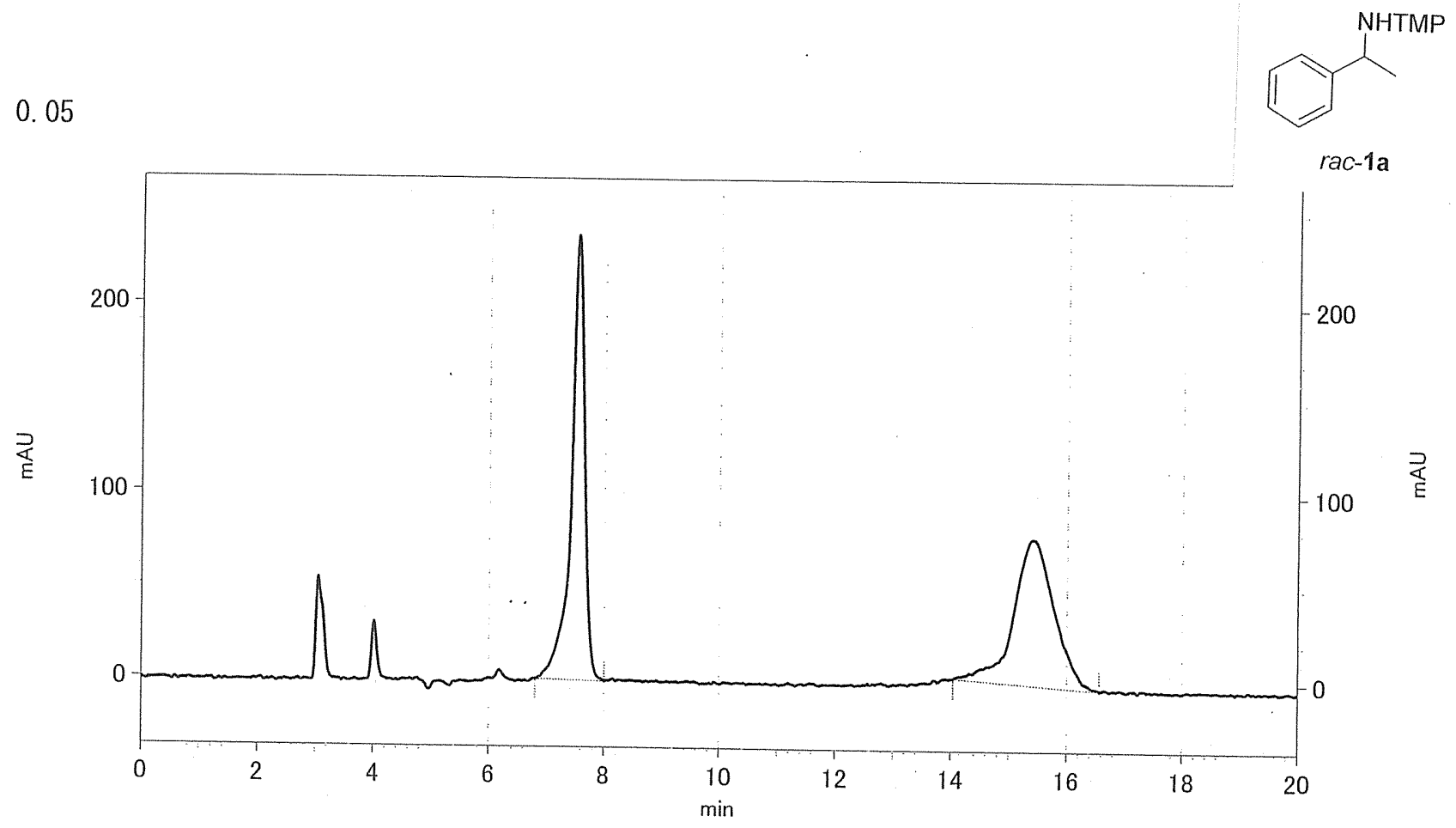

\begin{tabular}{ccccc} 
Peak \# & Retention time & Type & Area & Area \% \\
\hline 1 & 7.53 & 11 & 15318359 & 49.975 \\
2 & 15.41 & BI & 15333771 & 50.025
\end{tabular}




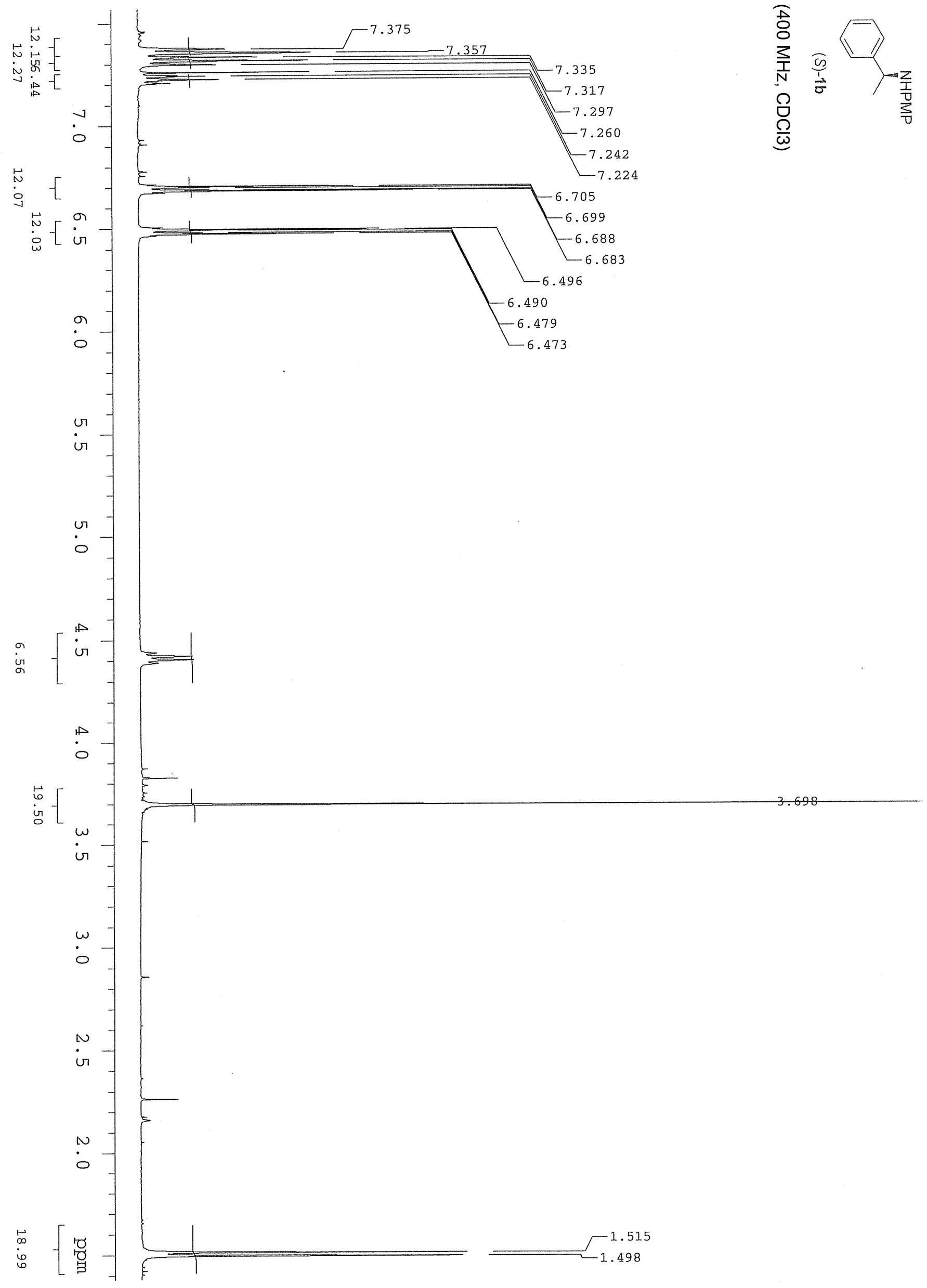




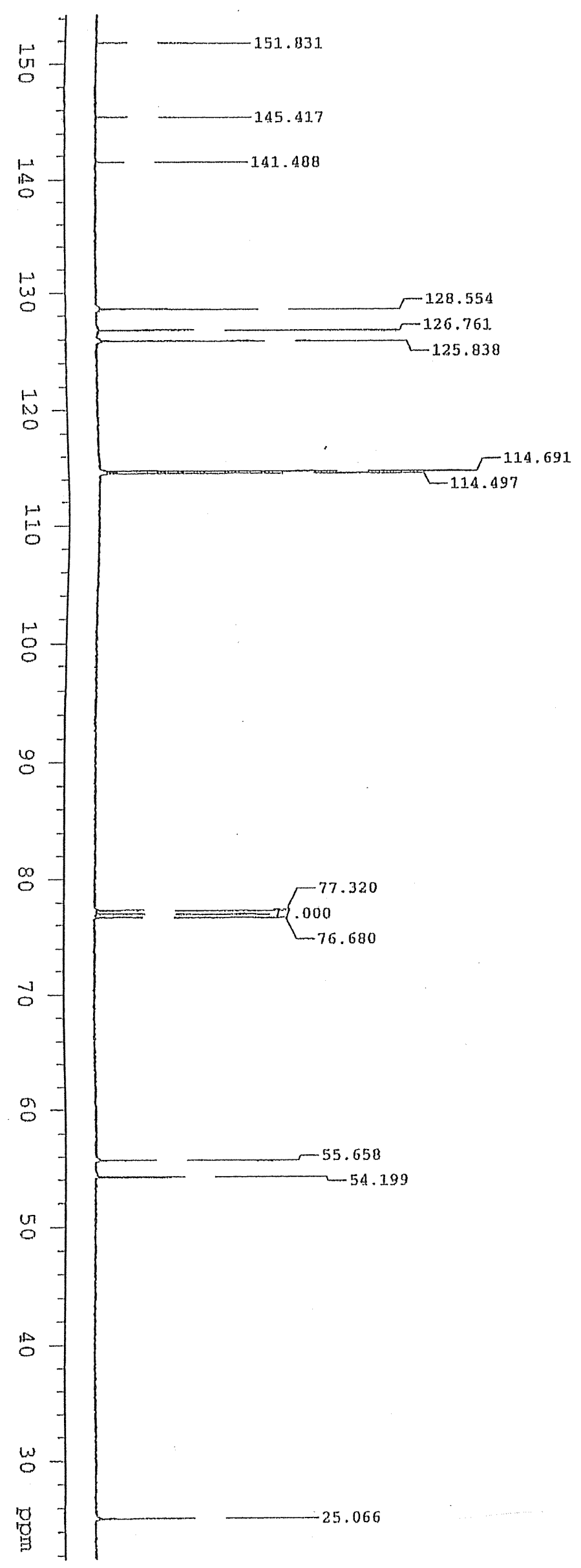

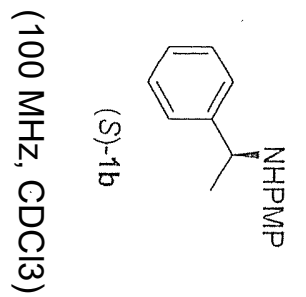


0.05

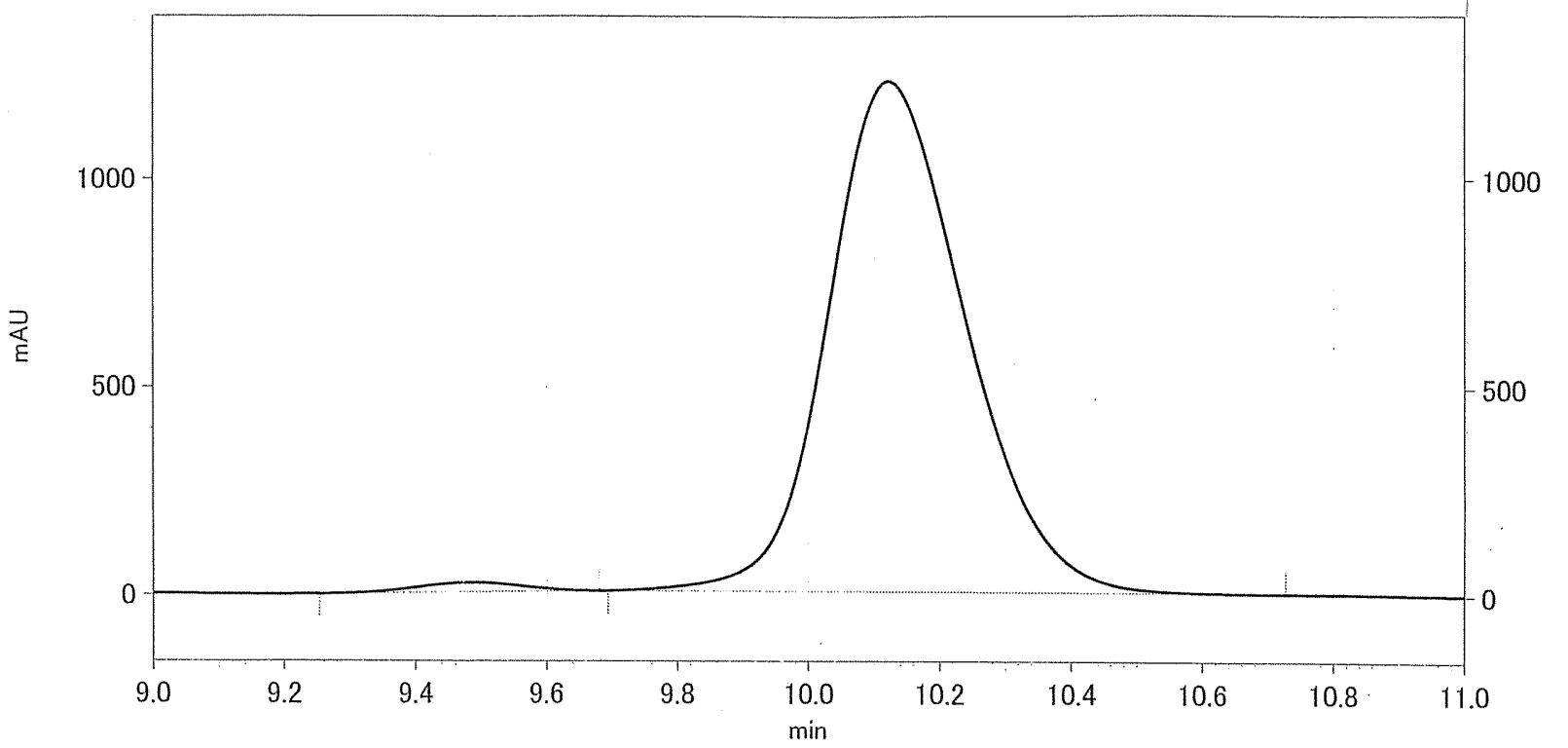

\begin{tabular}{ccccc} 
Peak \# & Retention time & Type & Area & Area \% \\
\hline 1 & 9.49 & $B 1$ & 1030775 & 1.422 \\
2 & 10.12 & BB & 71471799 & 98.578
\end{tabular}

0.05

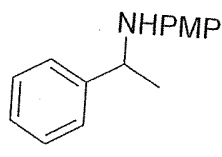

$r a c-1 b$

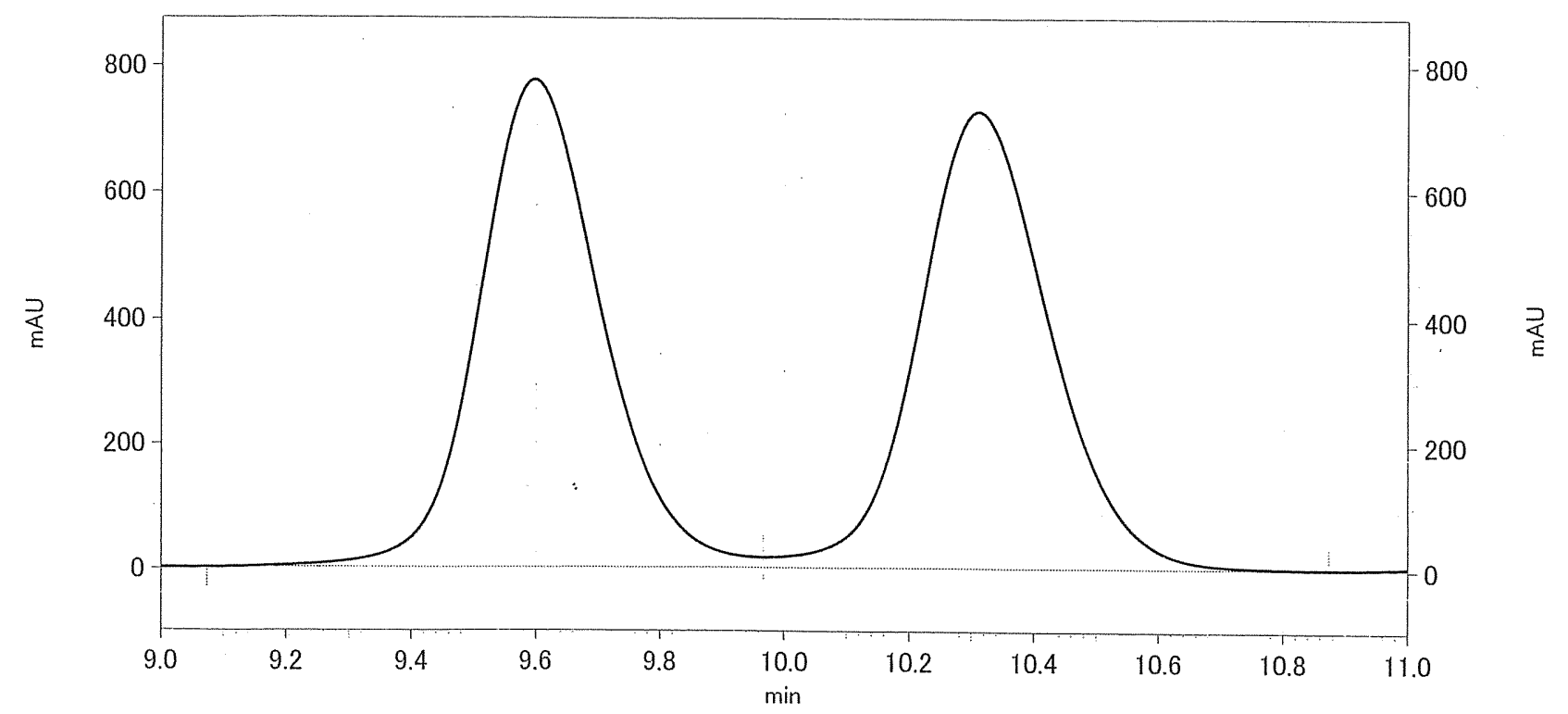

Peak \# Retention time Type
BV
VB Area Area \% 1
2 9.60 


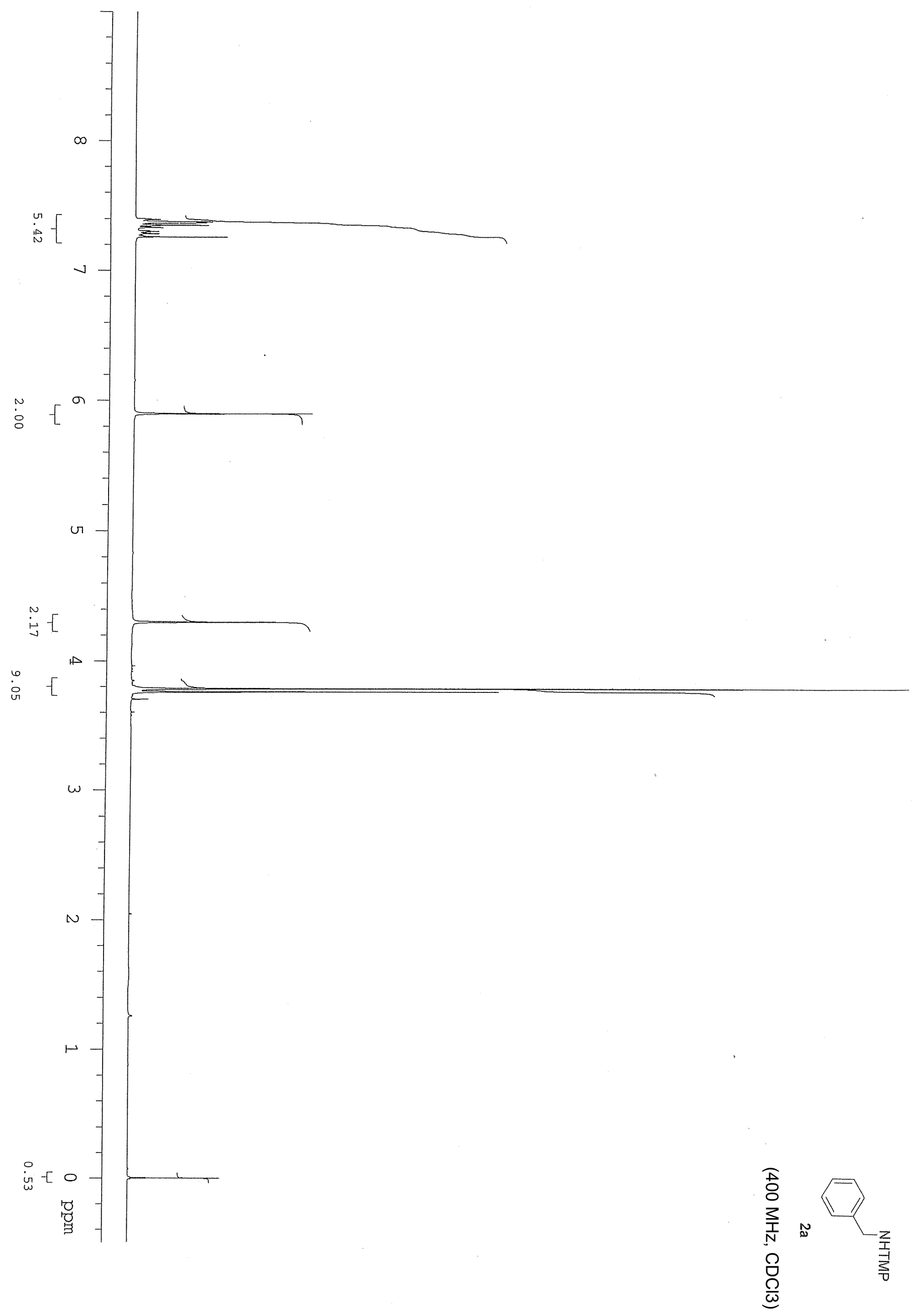




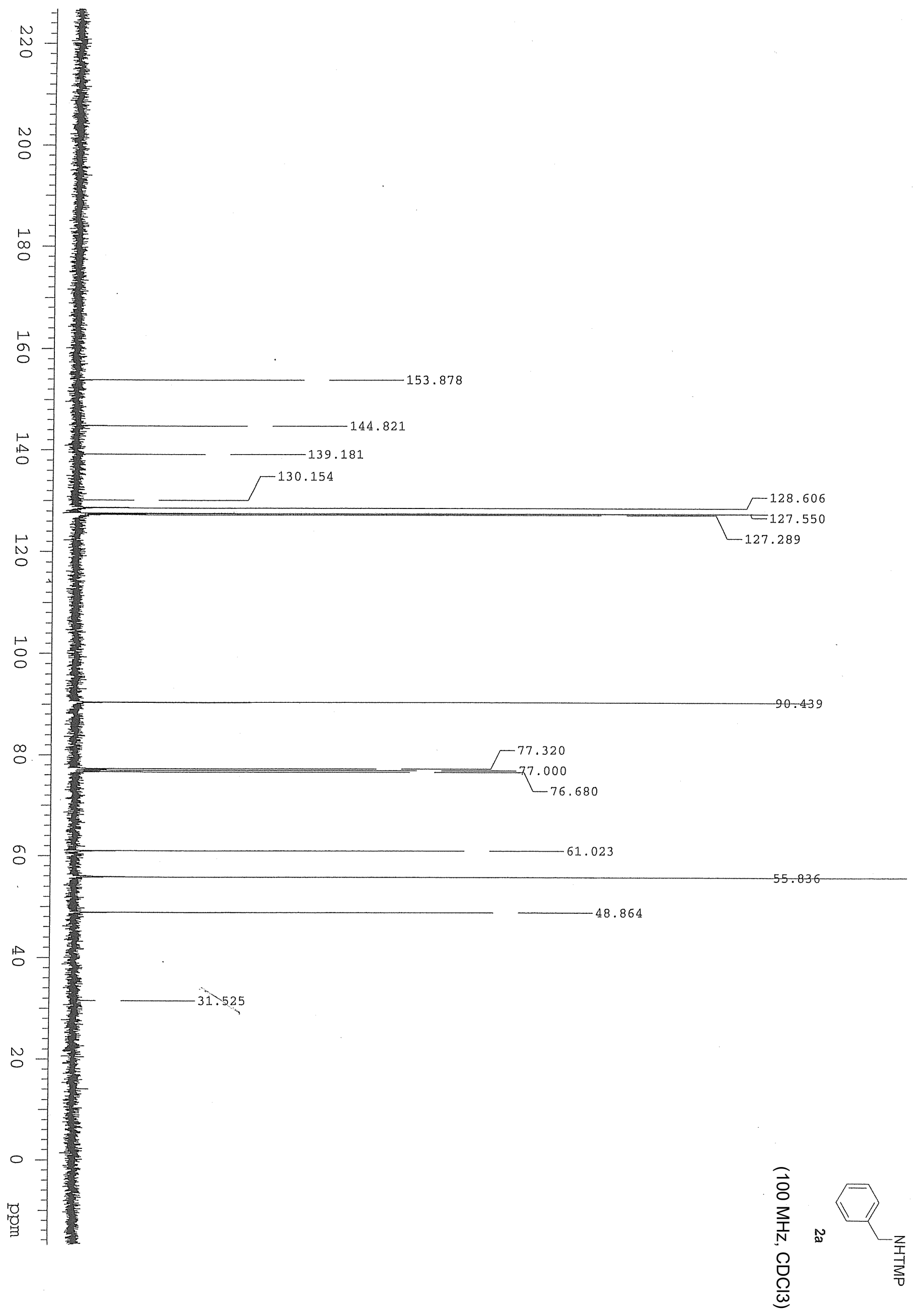




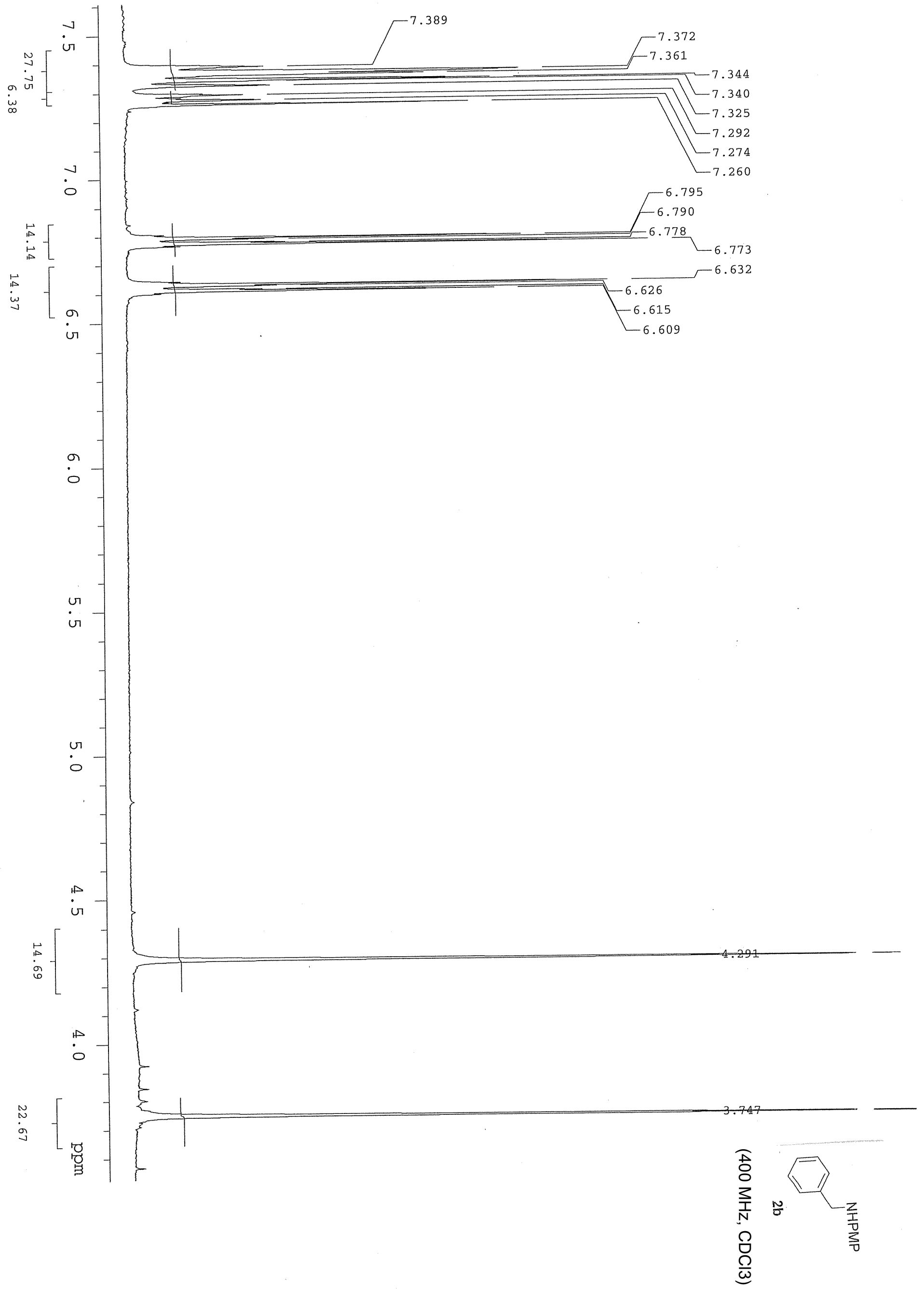




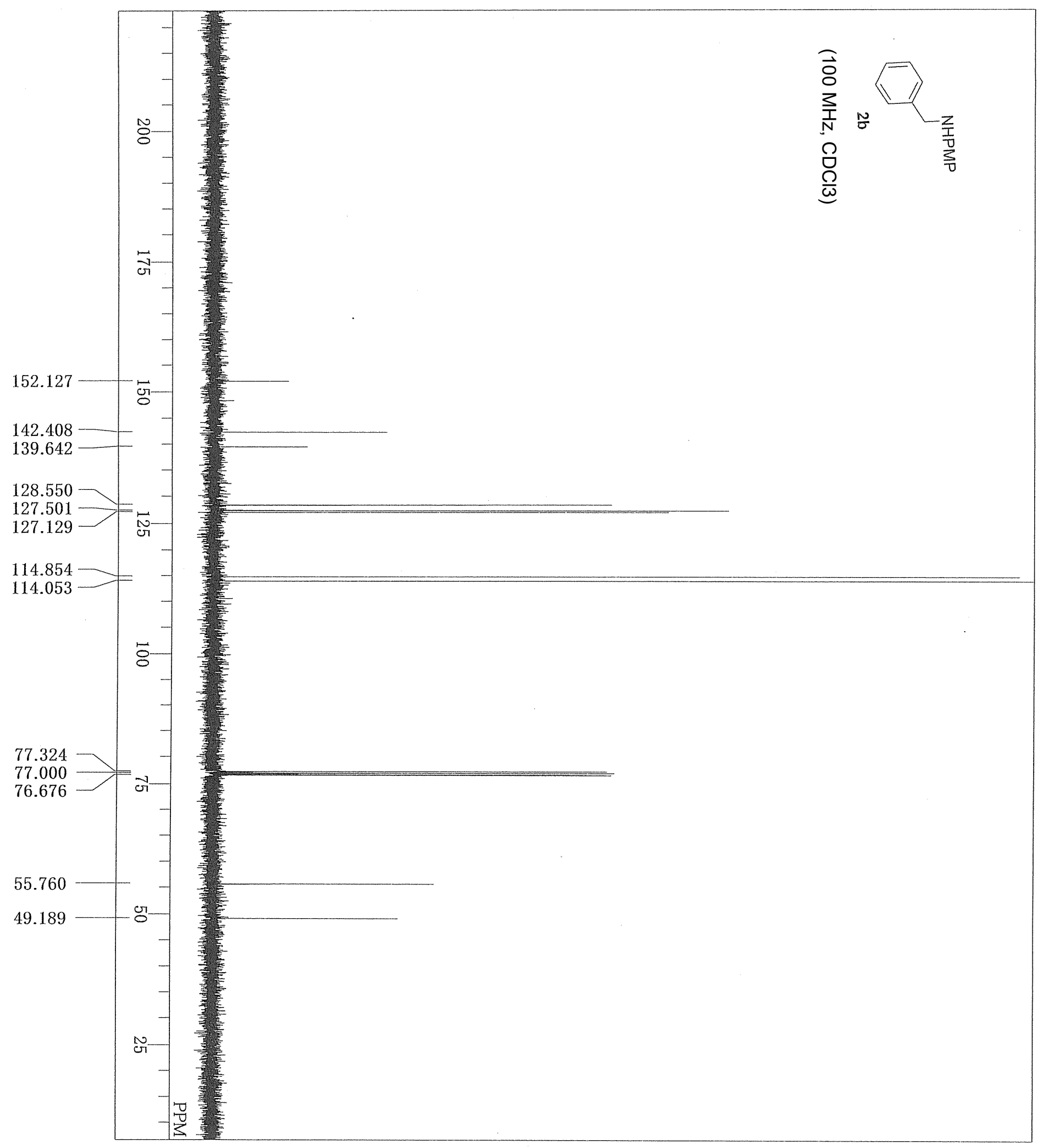

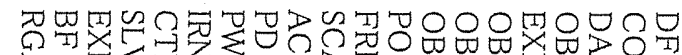

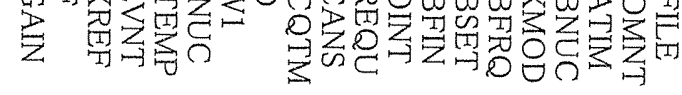

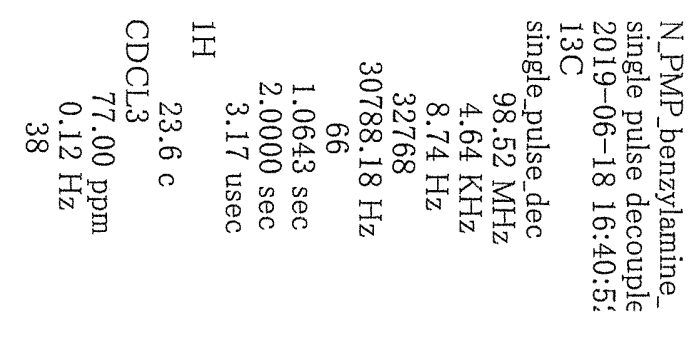




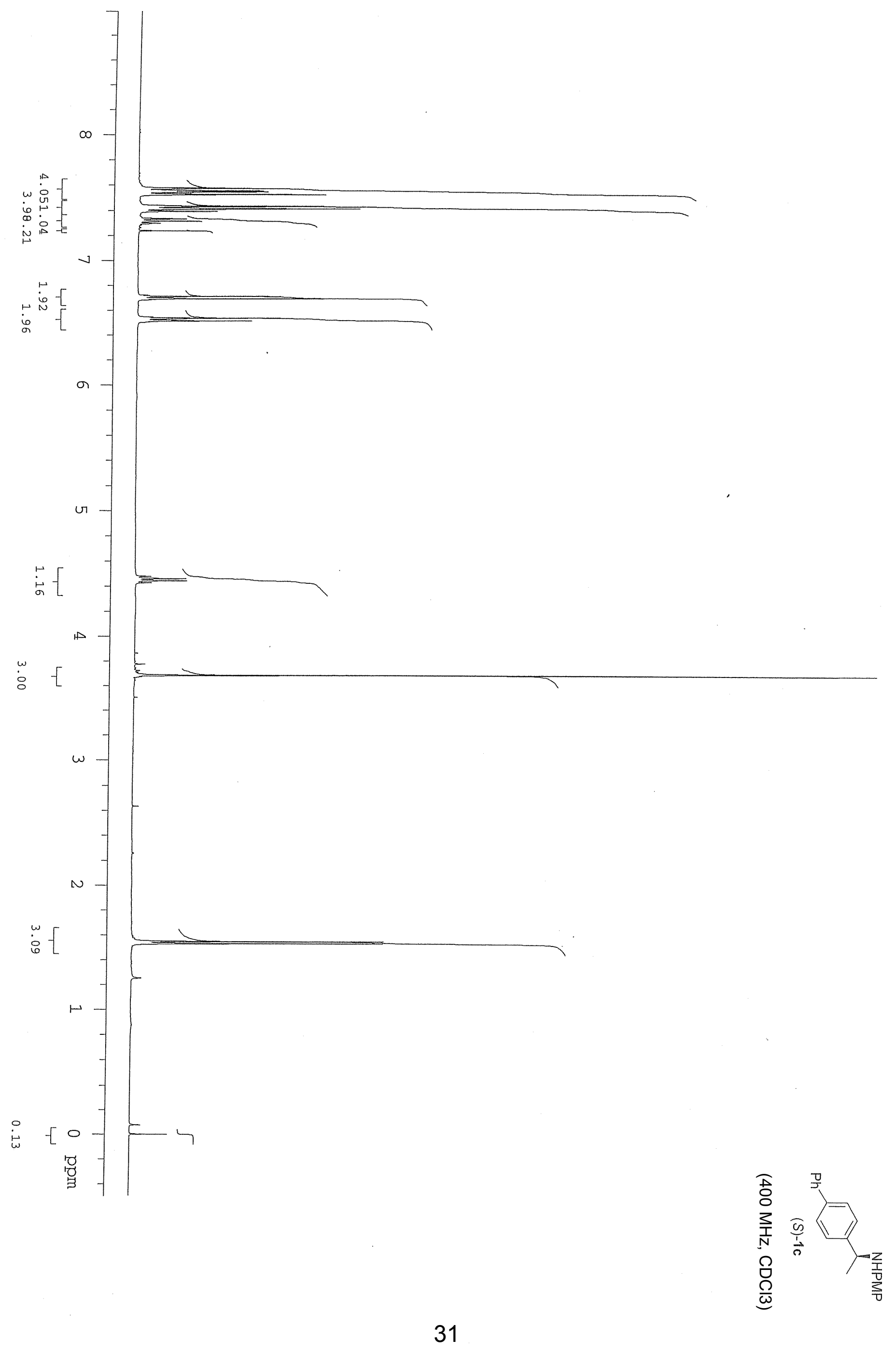




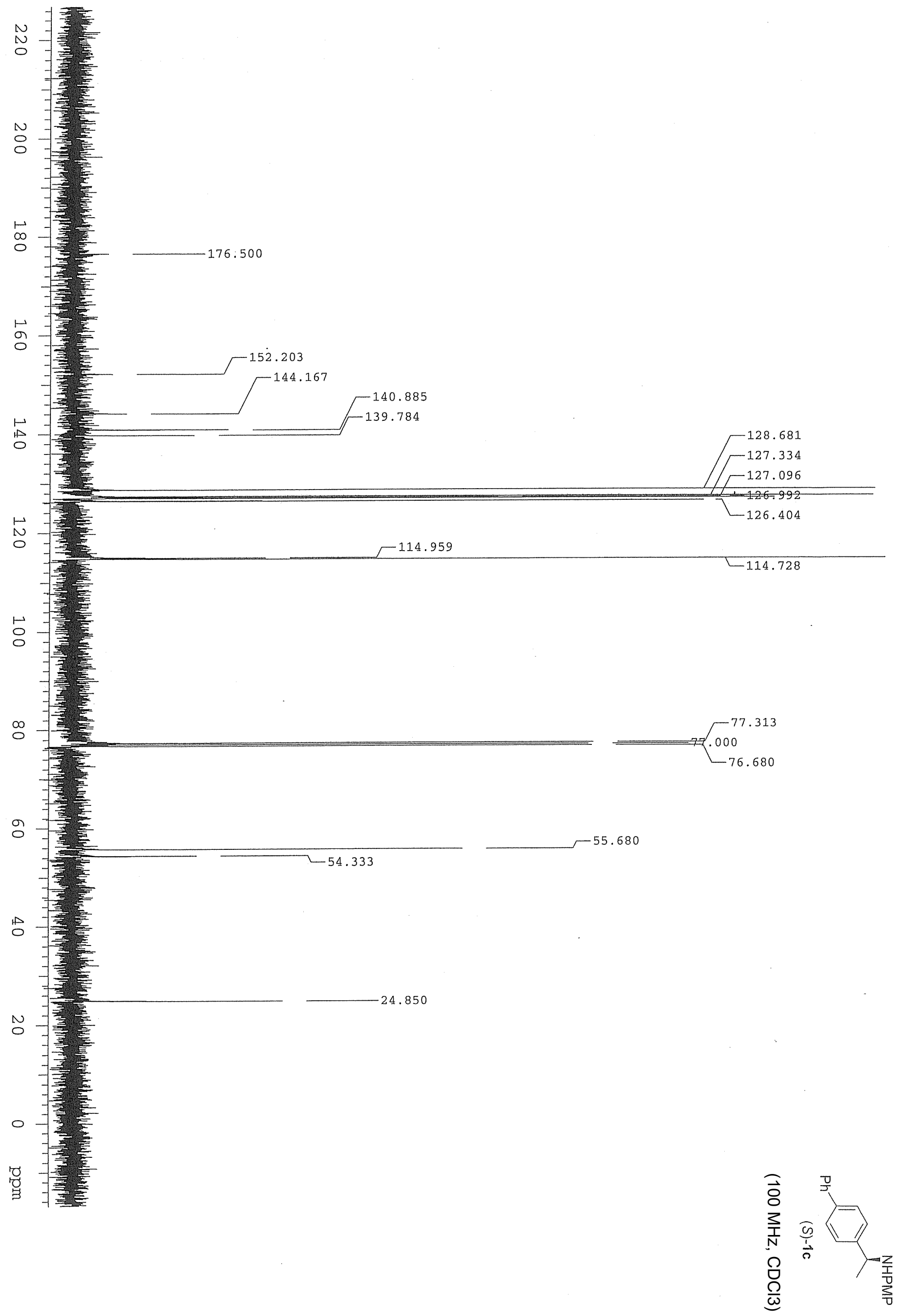




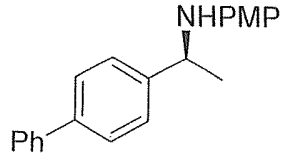

(S)-1c

0.05

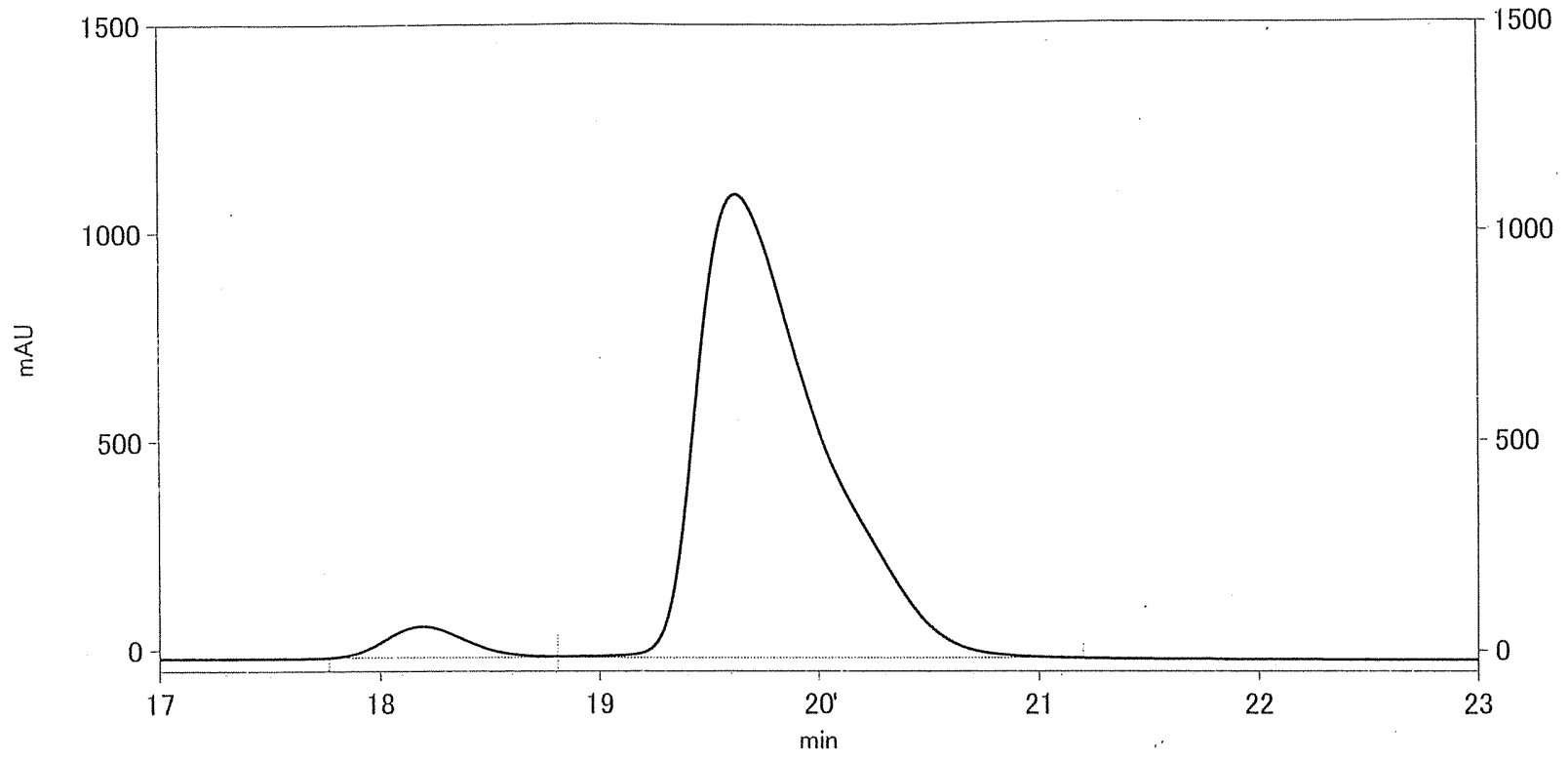

\begin{tabular}{ccccc} 
Peak \# & Retention time & Type & Area & Area \% \\
\hline 1 & 18.19 & BV & 7793919 & 4.433 \\
2 & 19.63 & VB & 168004709 & 95.567
\end{tabular}

0.05
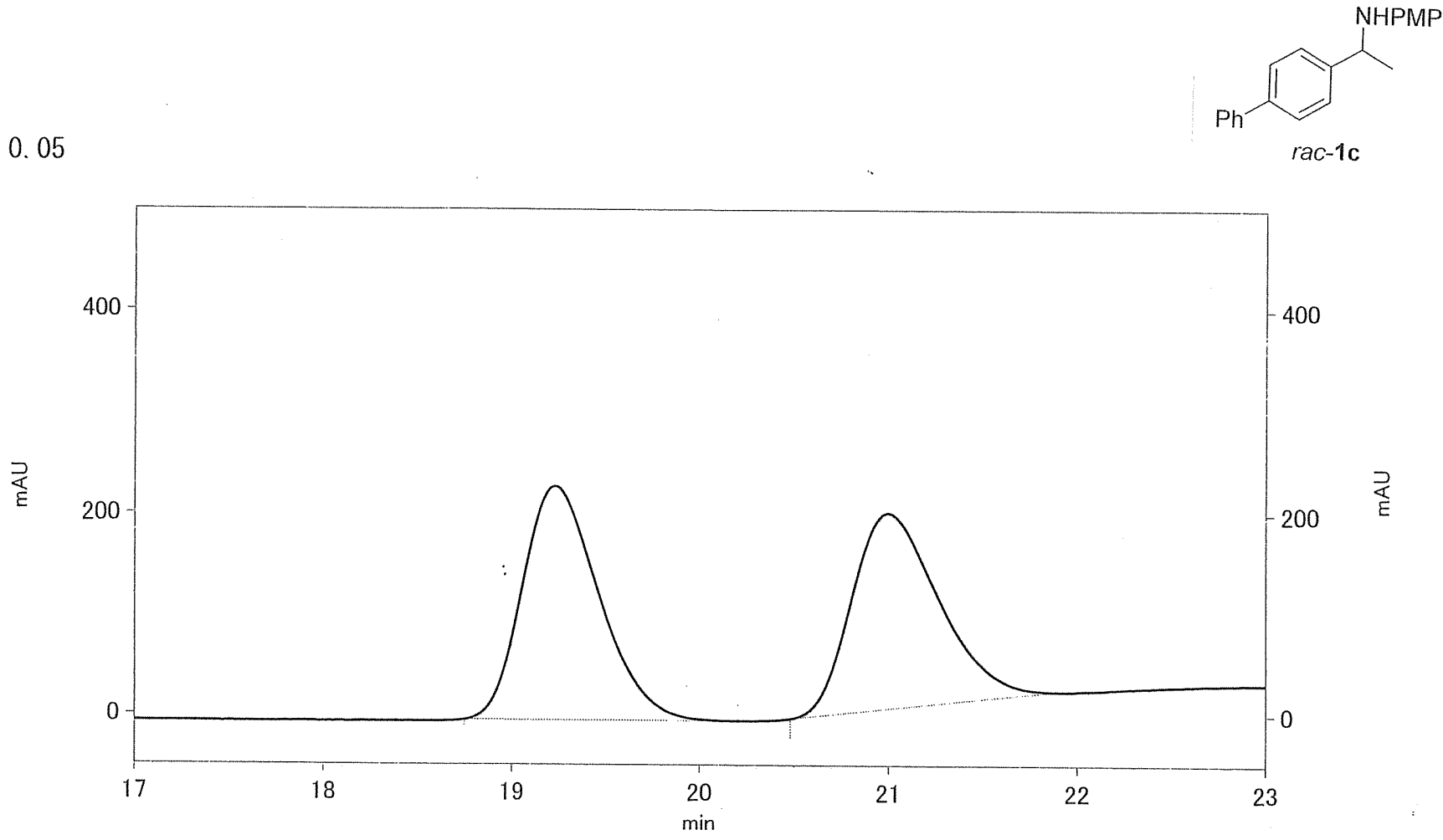

Peak \# 1
2 


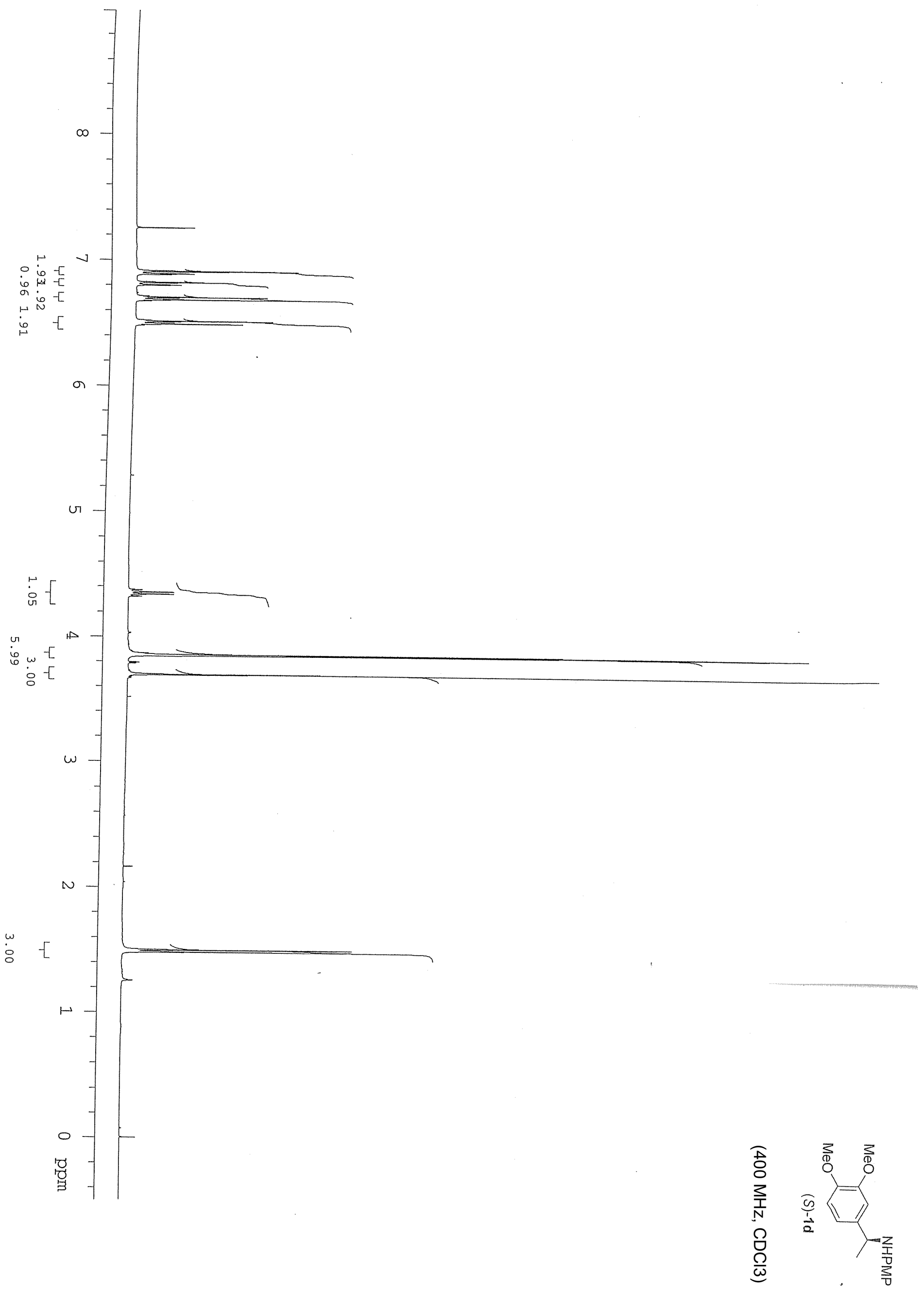




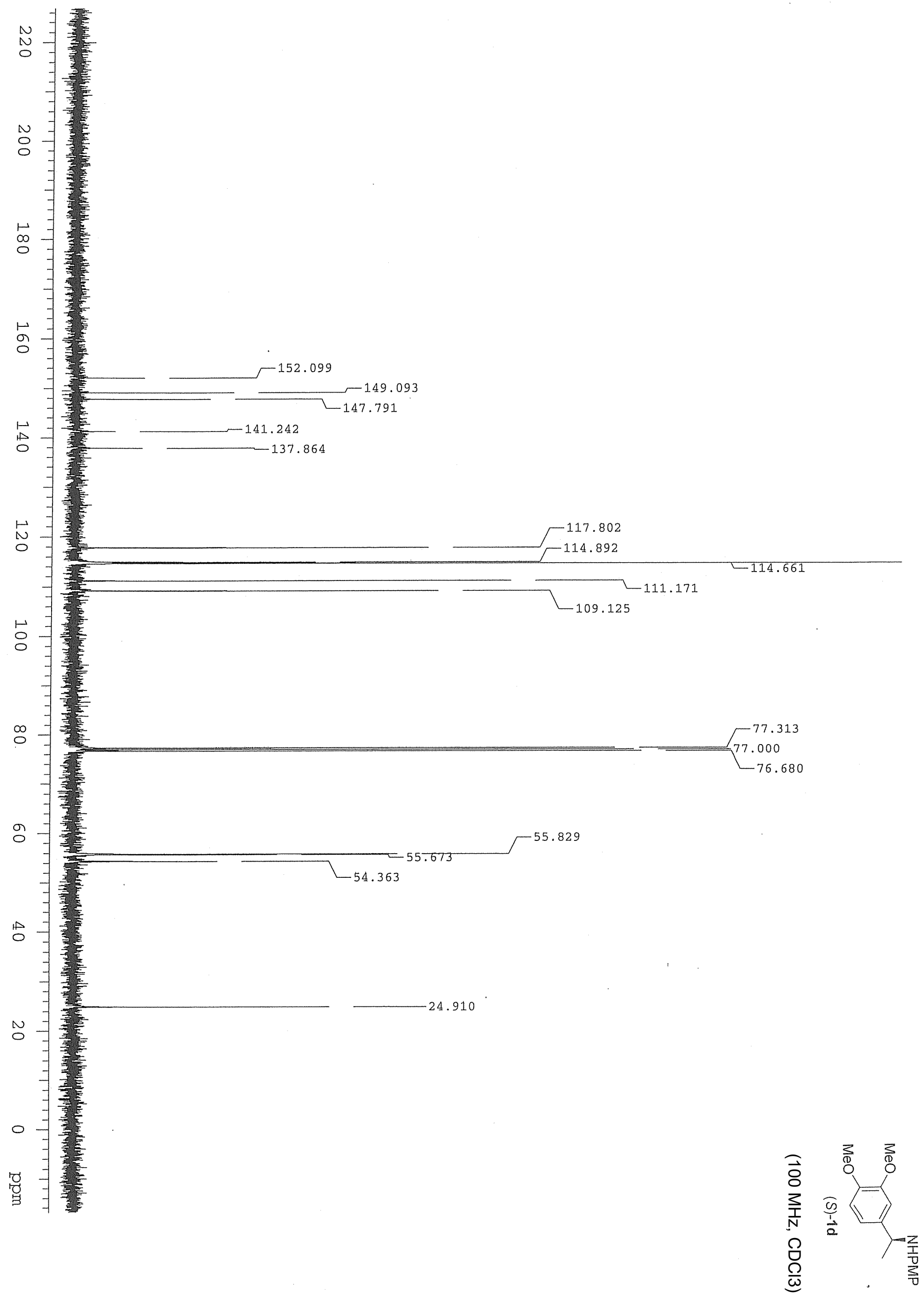




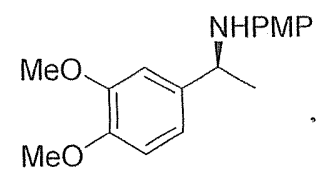

(S)-1d

0.05

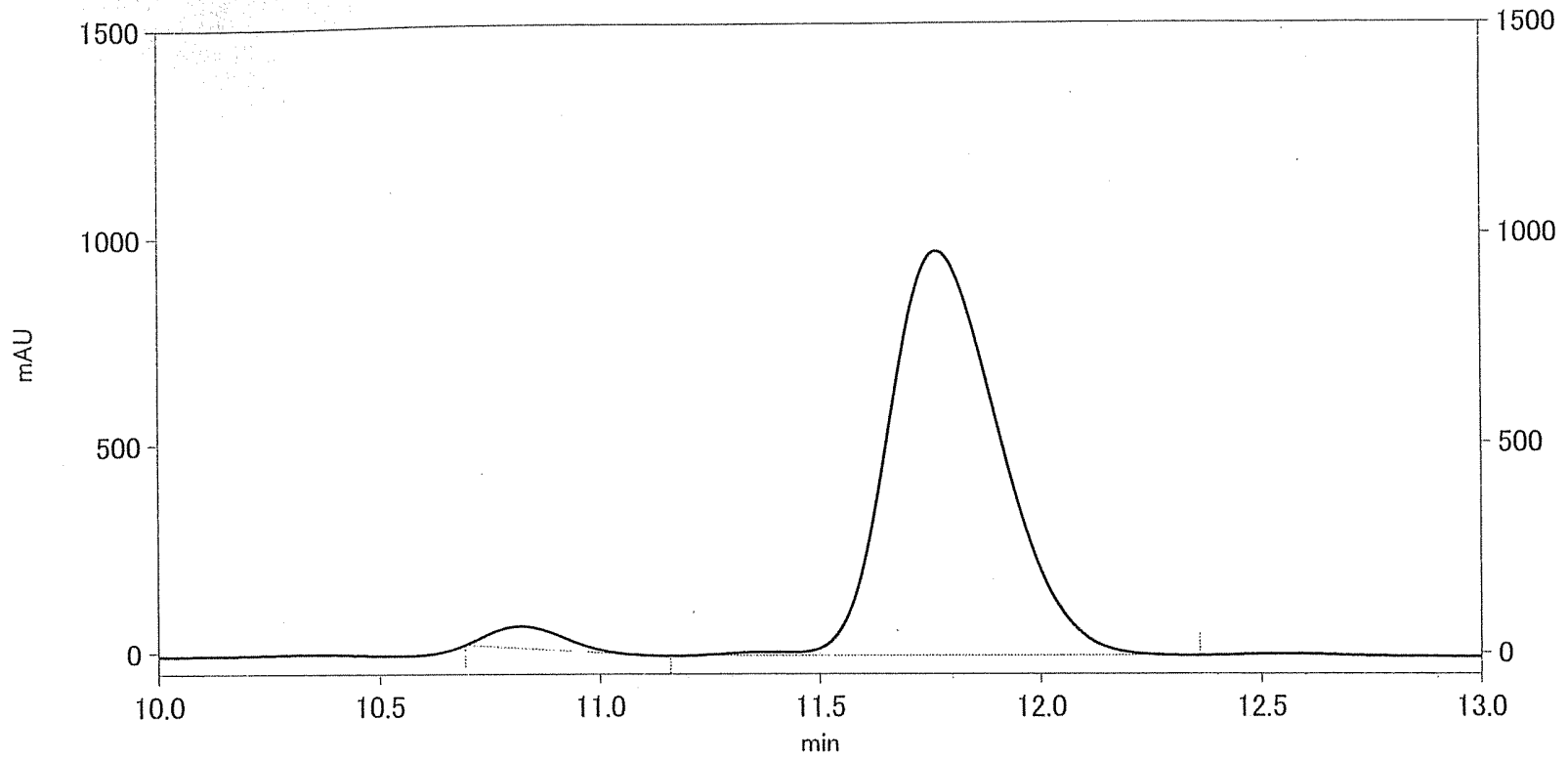

\begin{tabular}{ccccc} 
Peak \# & Retention time & Type & Area & Area \% \\
\hline 1 & 10.82 & BB & 2395554 & 3.438 \\
2 & 11.77 & BB & 67278096 & 96.562
\end{tabular}

0.05
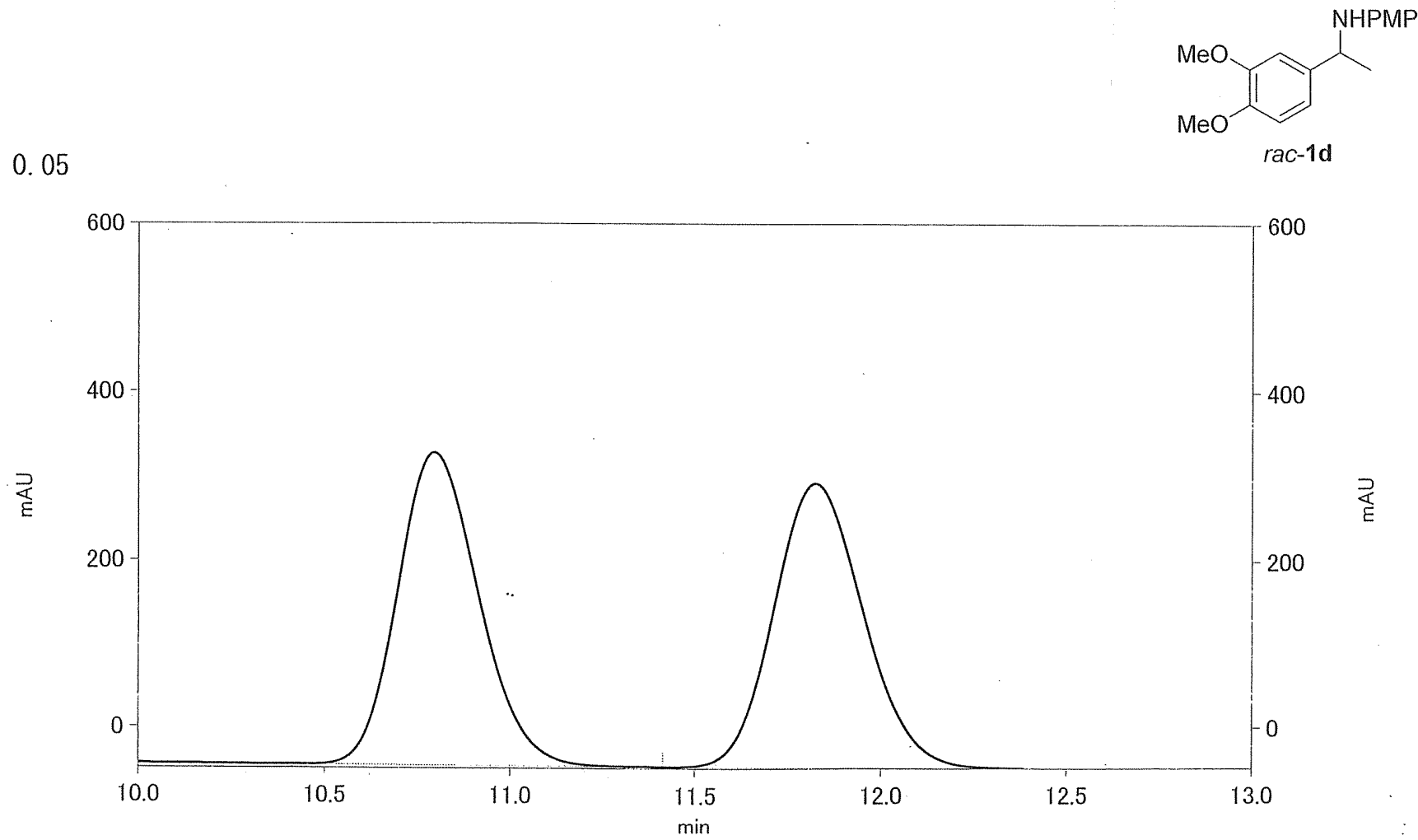

Peak \#

Retention time

Type

VB 


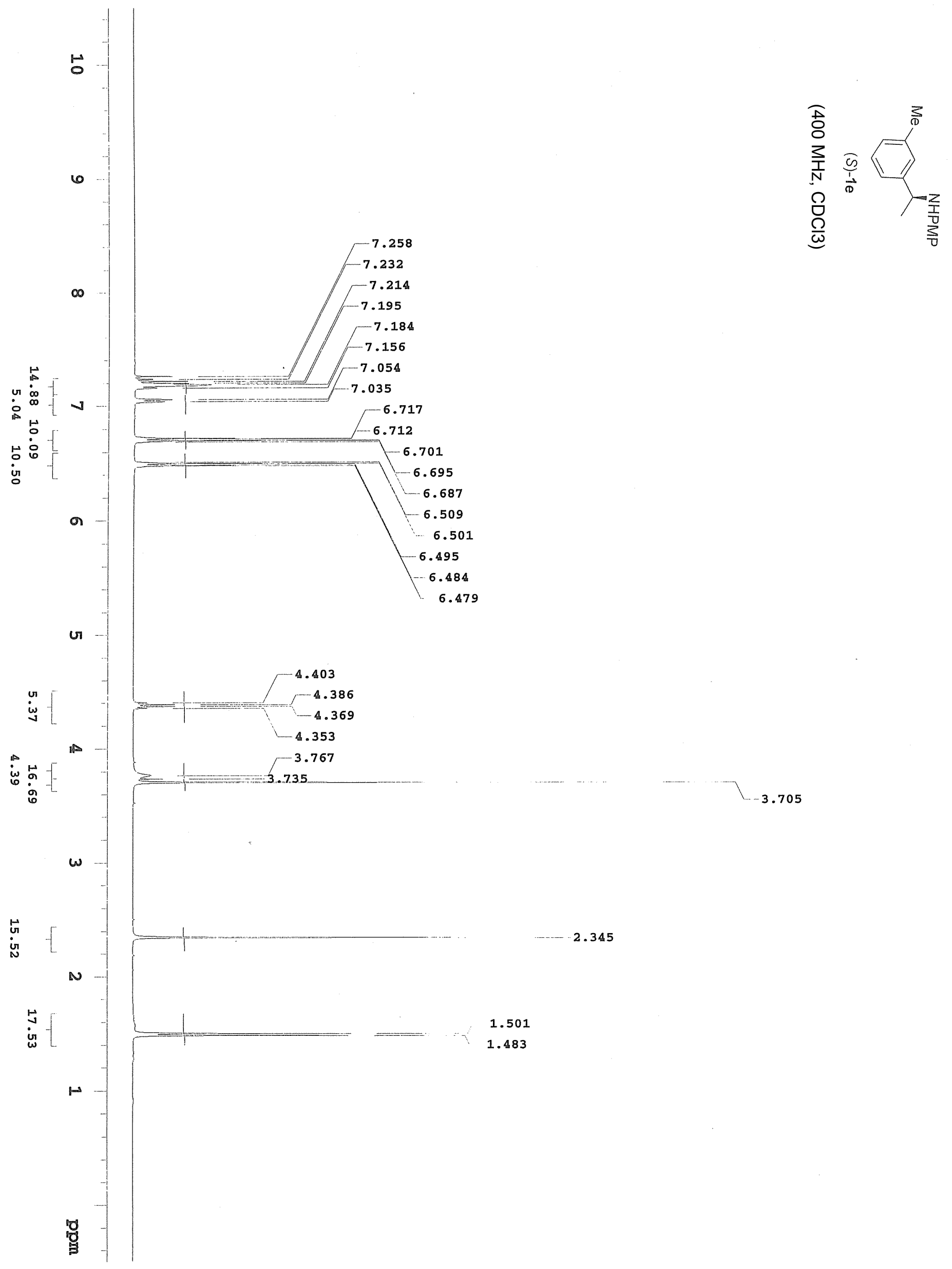



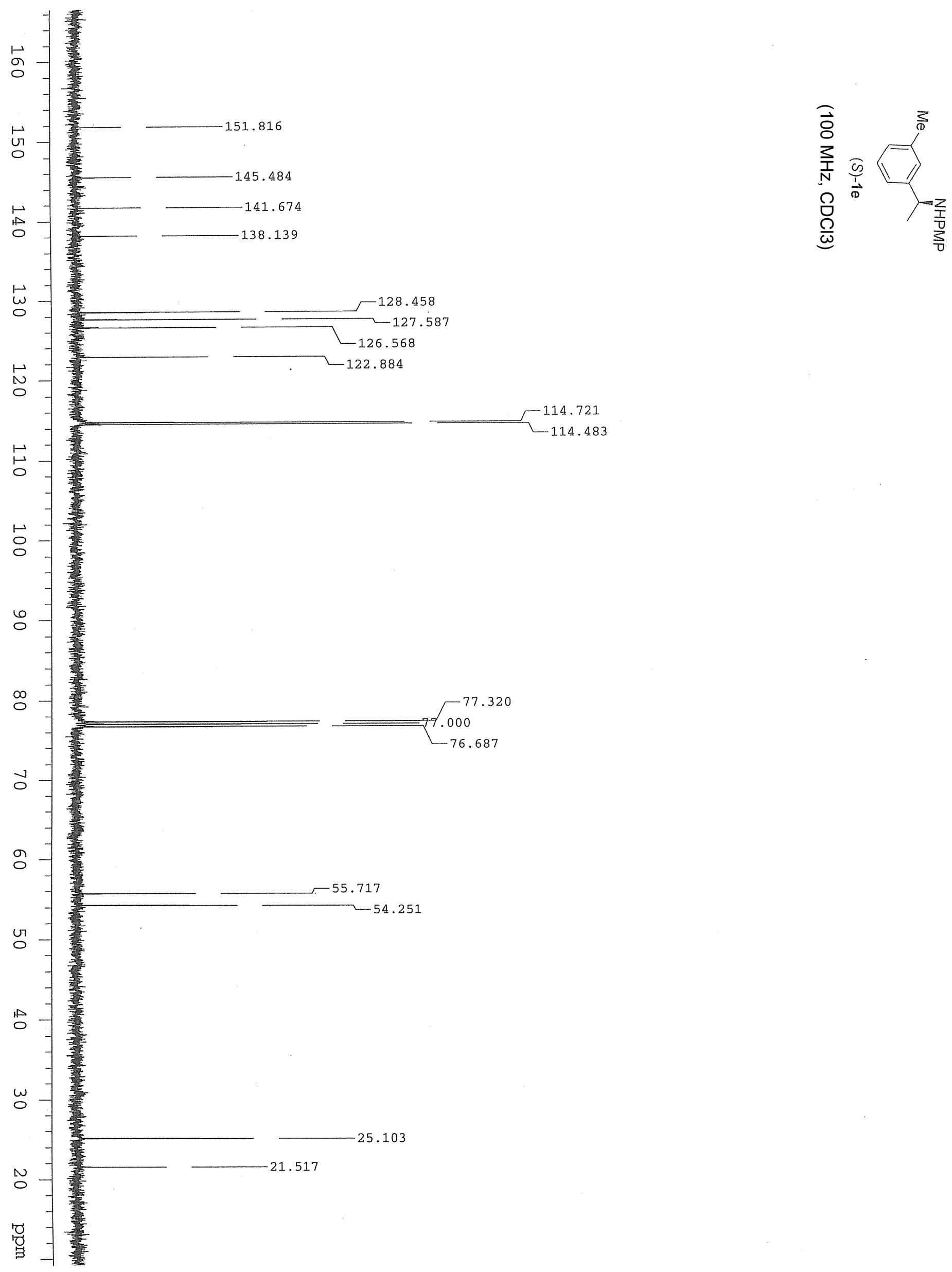
0.05
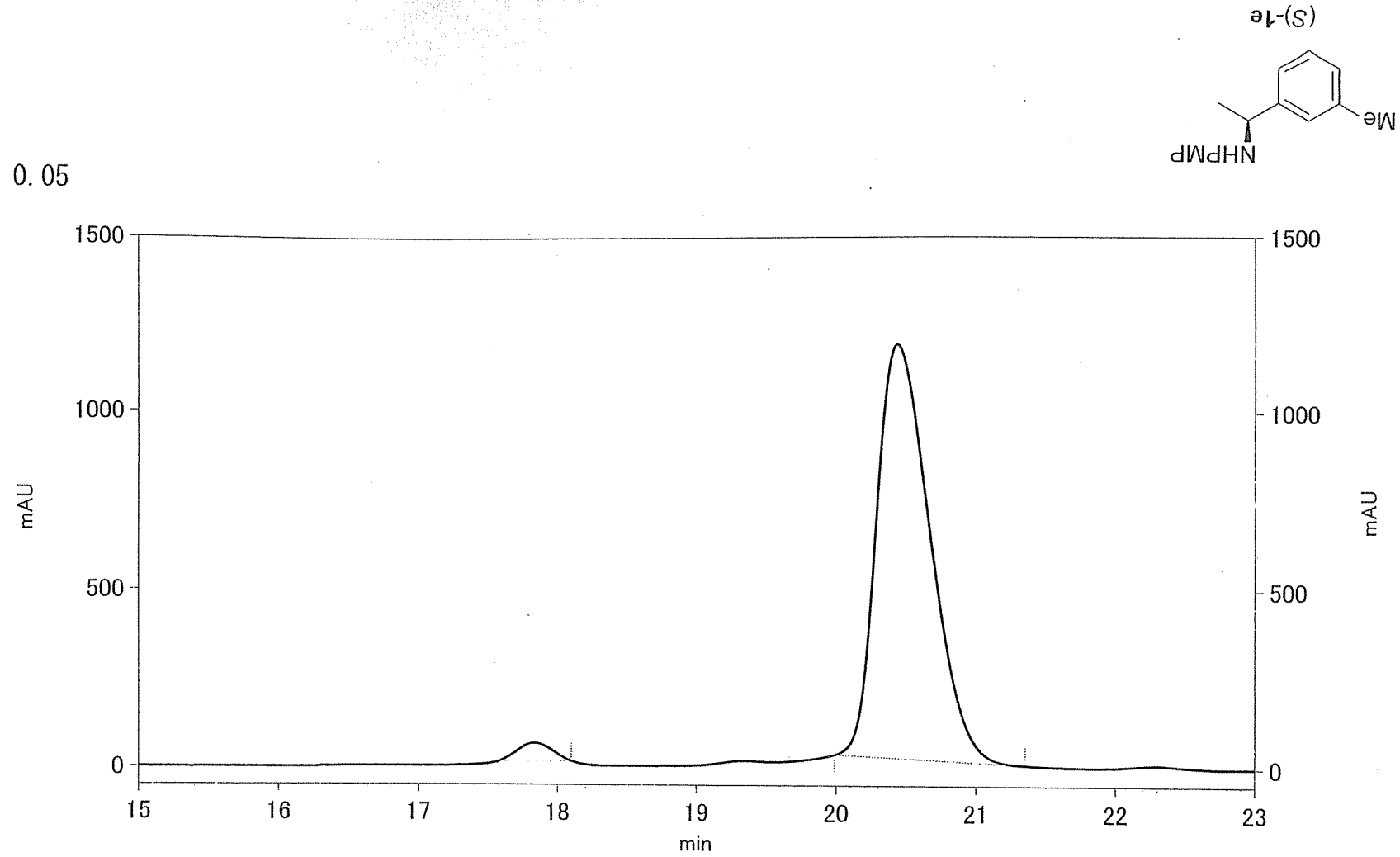

\begin{tabular}{ccccc} 
Peak \# & Retention time & Type & Area & Area \% \\
\hline 1 & 17.83 & $B 1$ & 3620092 & 2.836 \\
2 & 20.43 & BB & 124041176 & 97.164
\end{tabular}

0.05
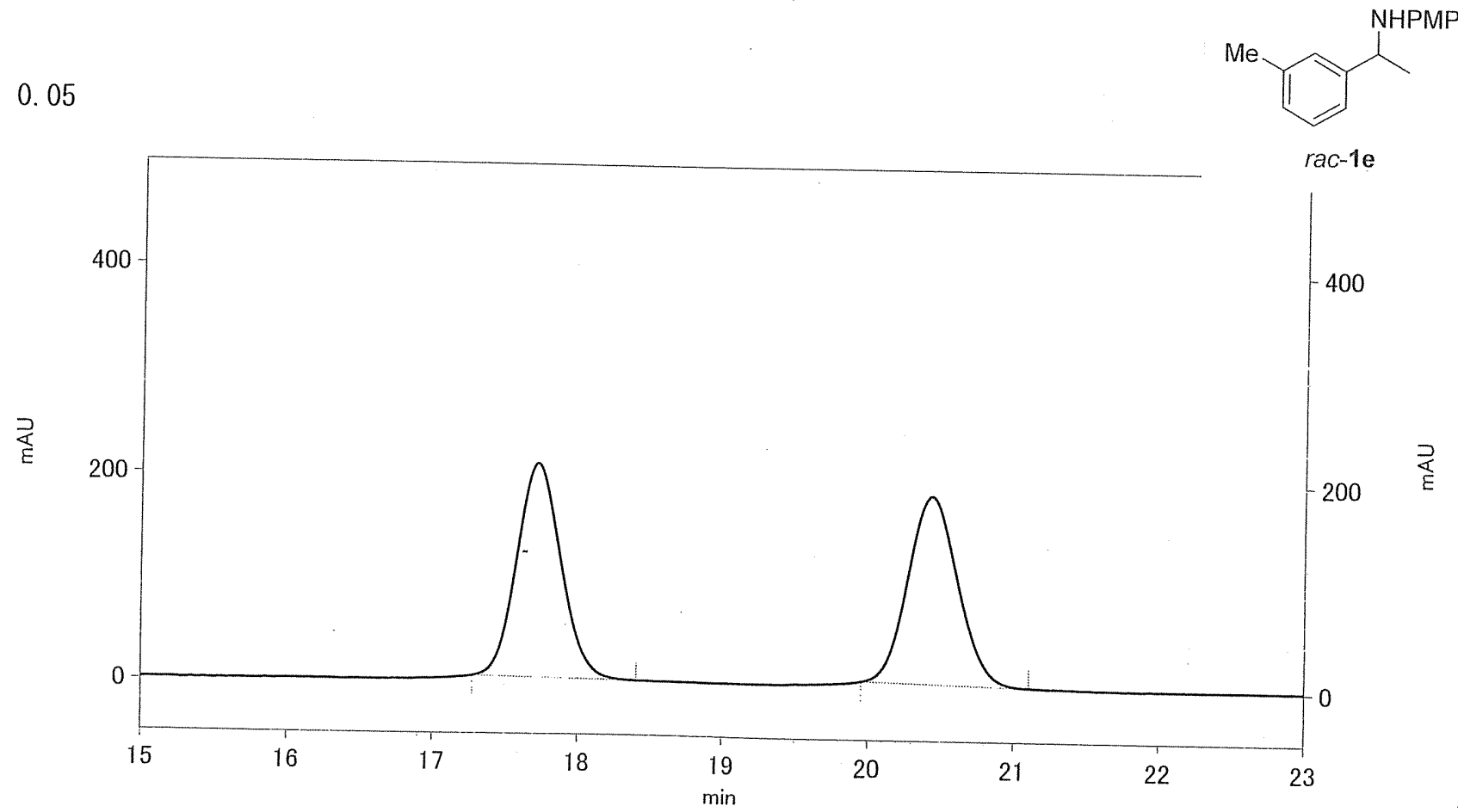

Peak \# 


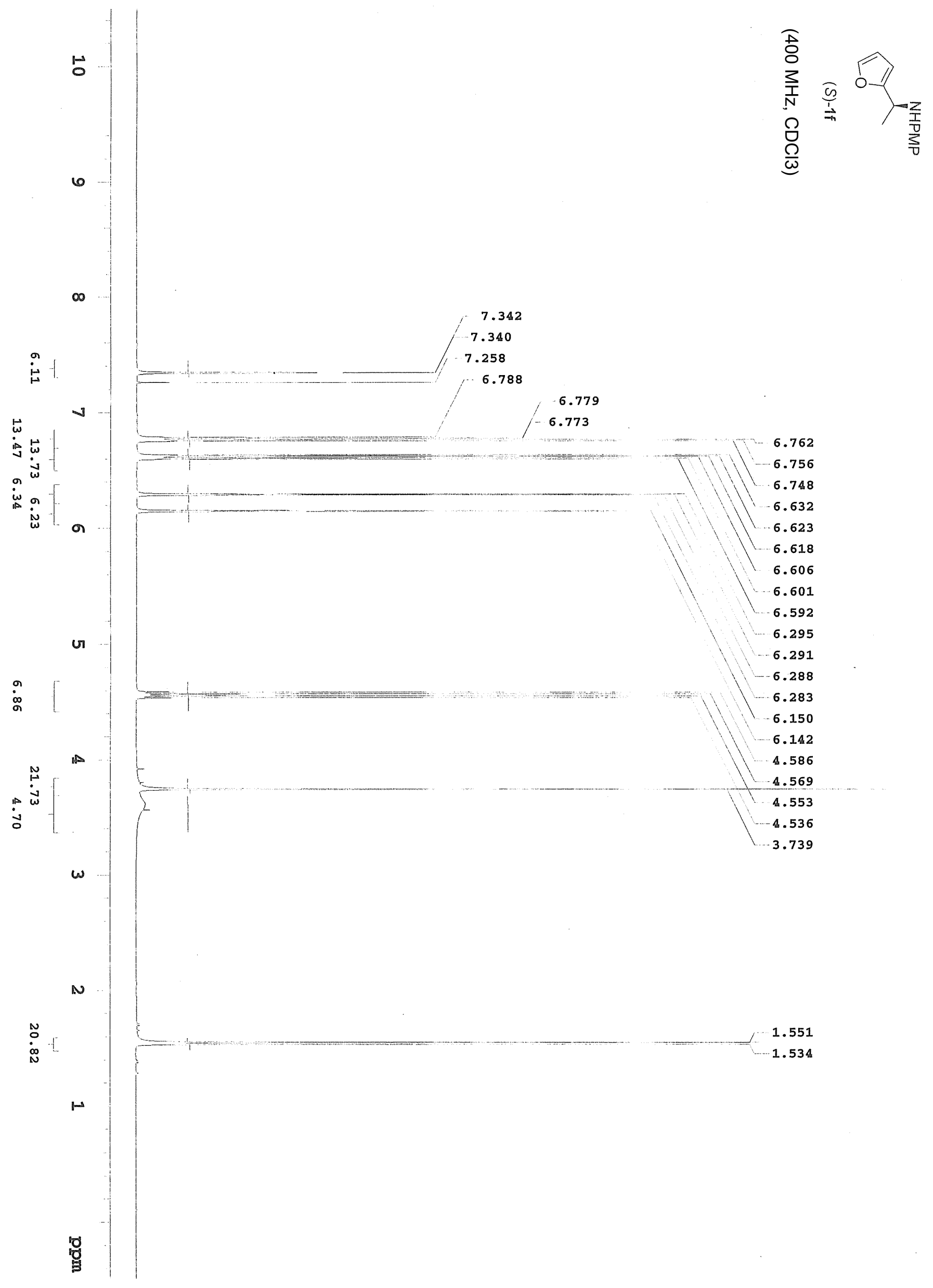




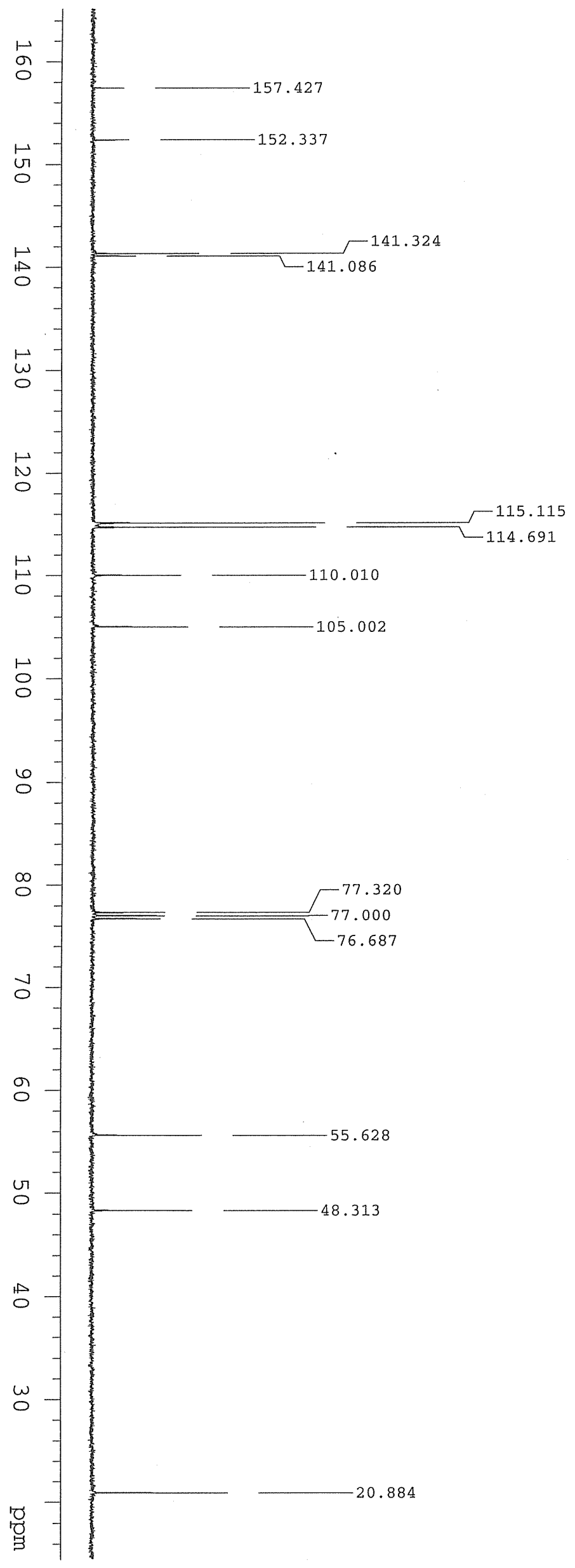


0.05
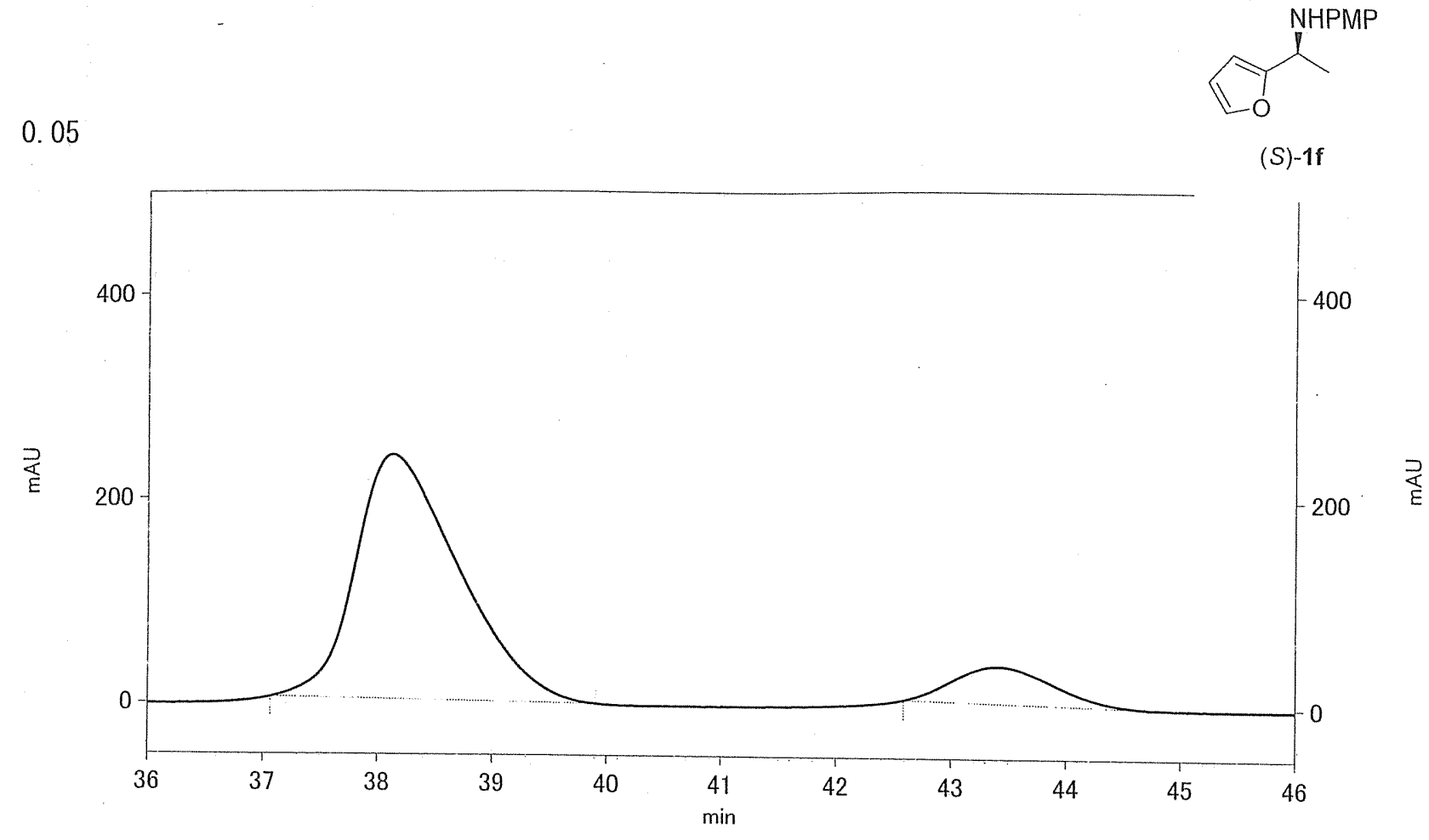

\begin{tabular}{ccccc} 
Peak \# & Retention time & Type & Area & Area \% \\
\hline 1 & 38.13 & $\mathrm{BB}$ & 59778393 & 87.308 \\
2 & 43.41 & $\mathrm{BB}$ & 8690115 & 12.692
\end{tabular}

0.05

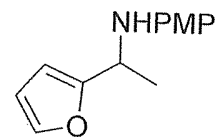

rac-1f

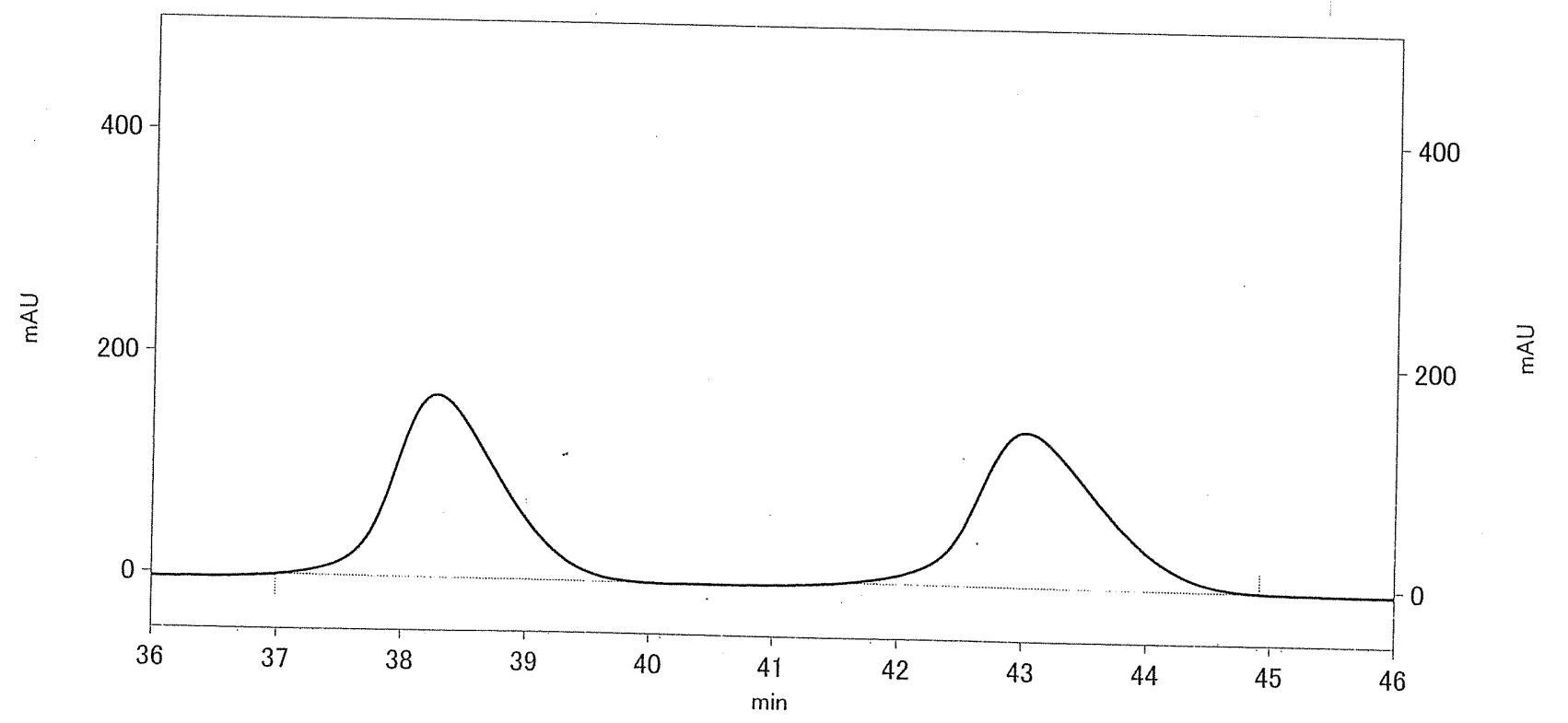

\begin{tabular}{ccccc} 
Peak \# & Retention time & Type & Area & Area \% \\
\hline 1 & 38.28 & BB & 40420383 & 50.440 \\
2 & 43.02 & $B B$ & 39715768 & 49.560
\end{tabular}




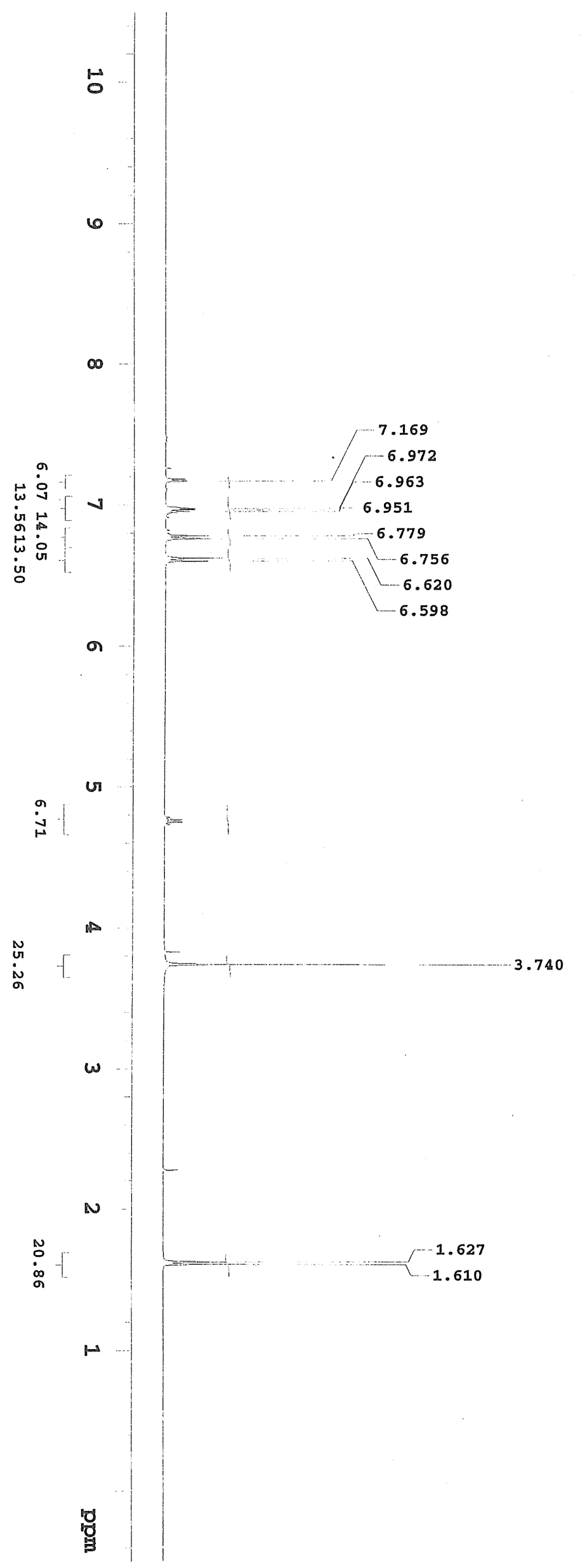

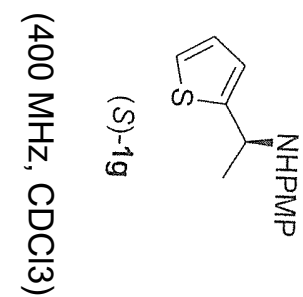



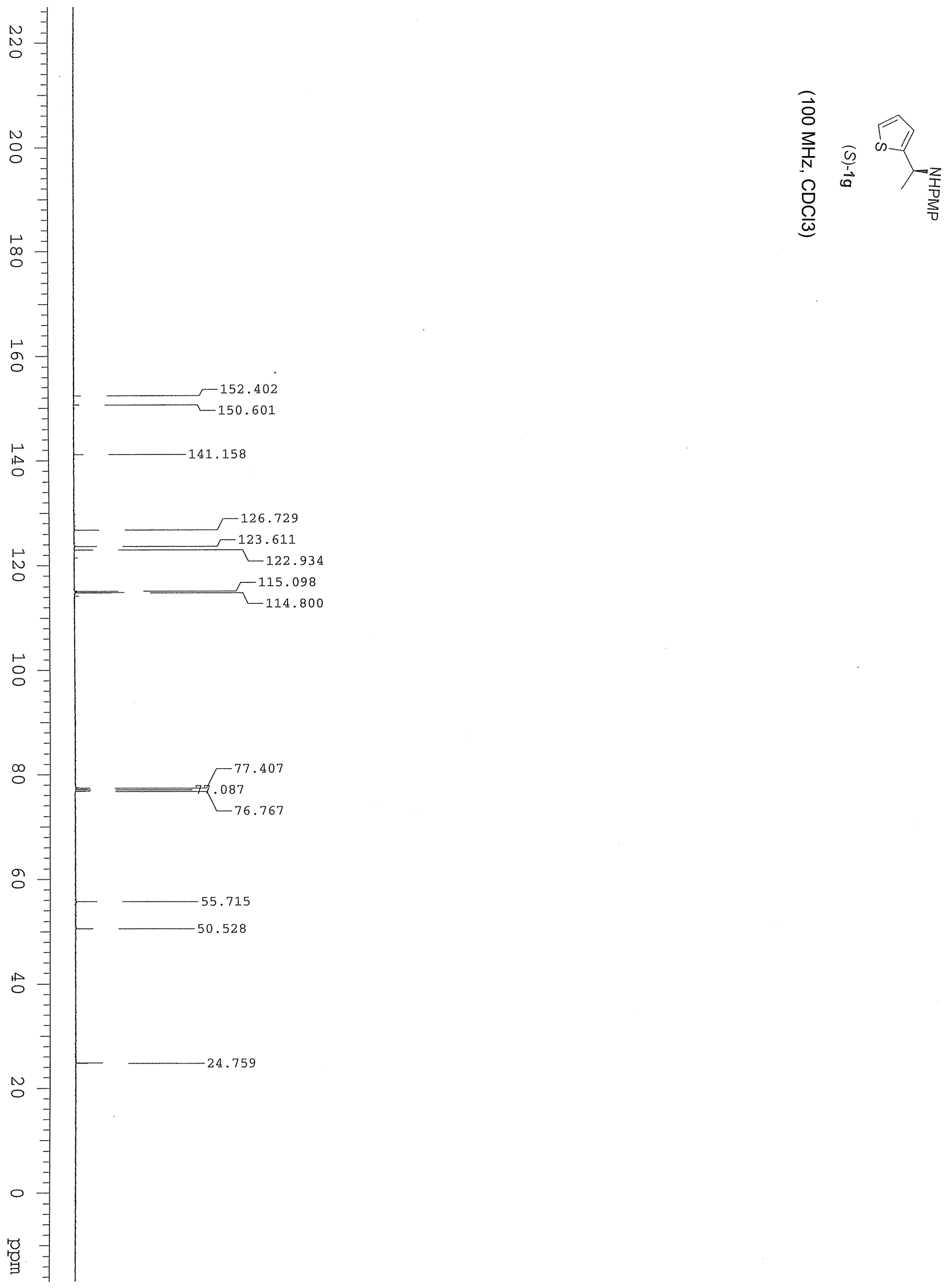
0.05

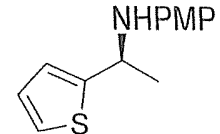

(S)-1g

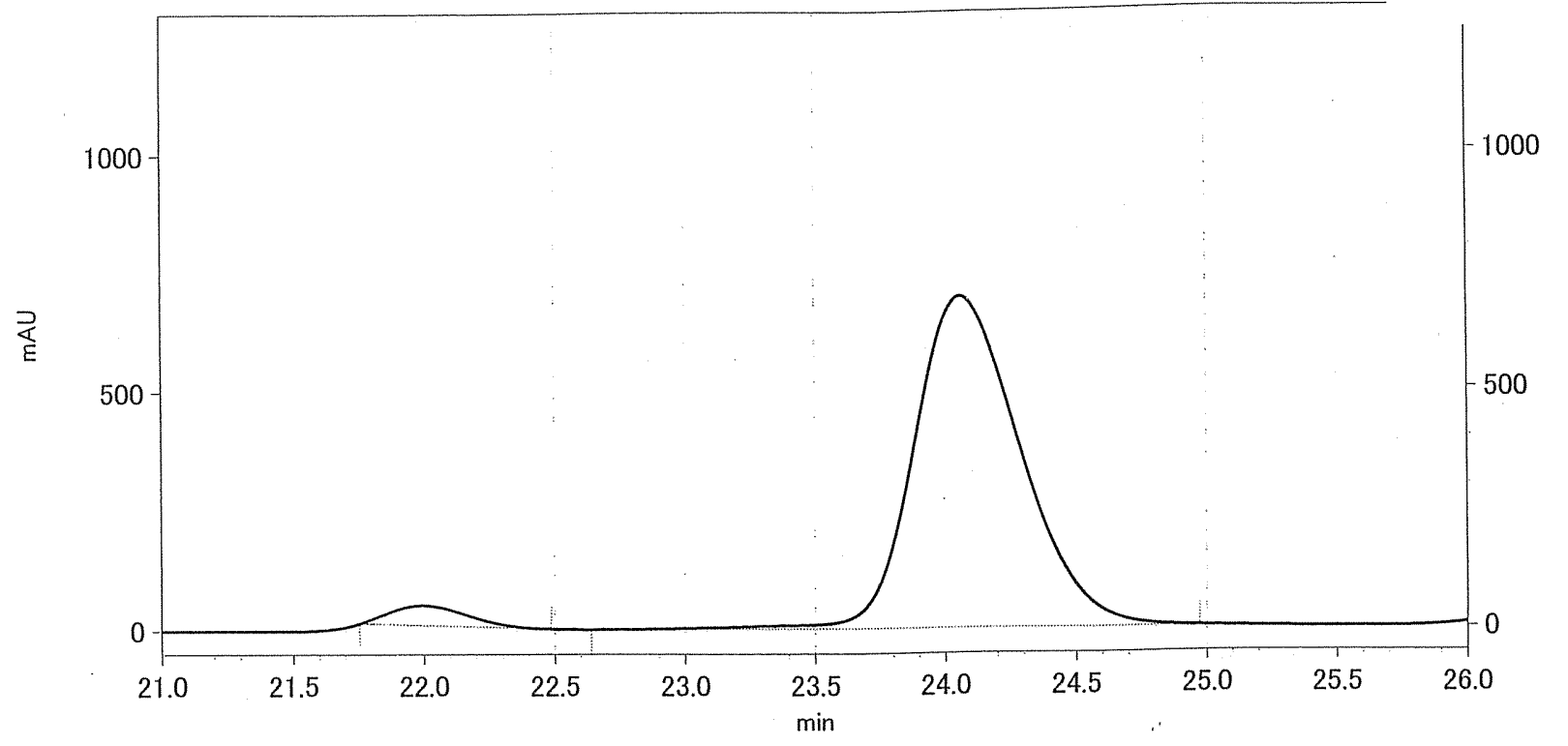

\begin{tabular}{ccccc} 
Peak \# & Retention time & Type & Area & Area \% \\
\hline 1 & 21.99 & BB & 3401455 & 4.134 \\
2 & 24.06 & BB & 78876157 & 95.866
\end{tabular}

0.05
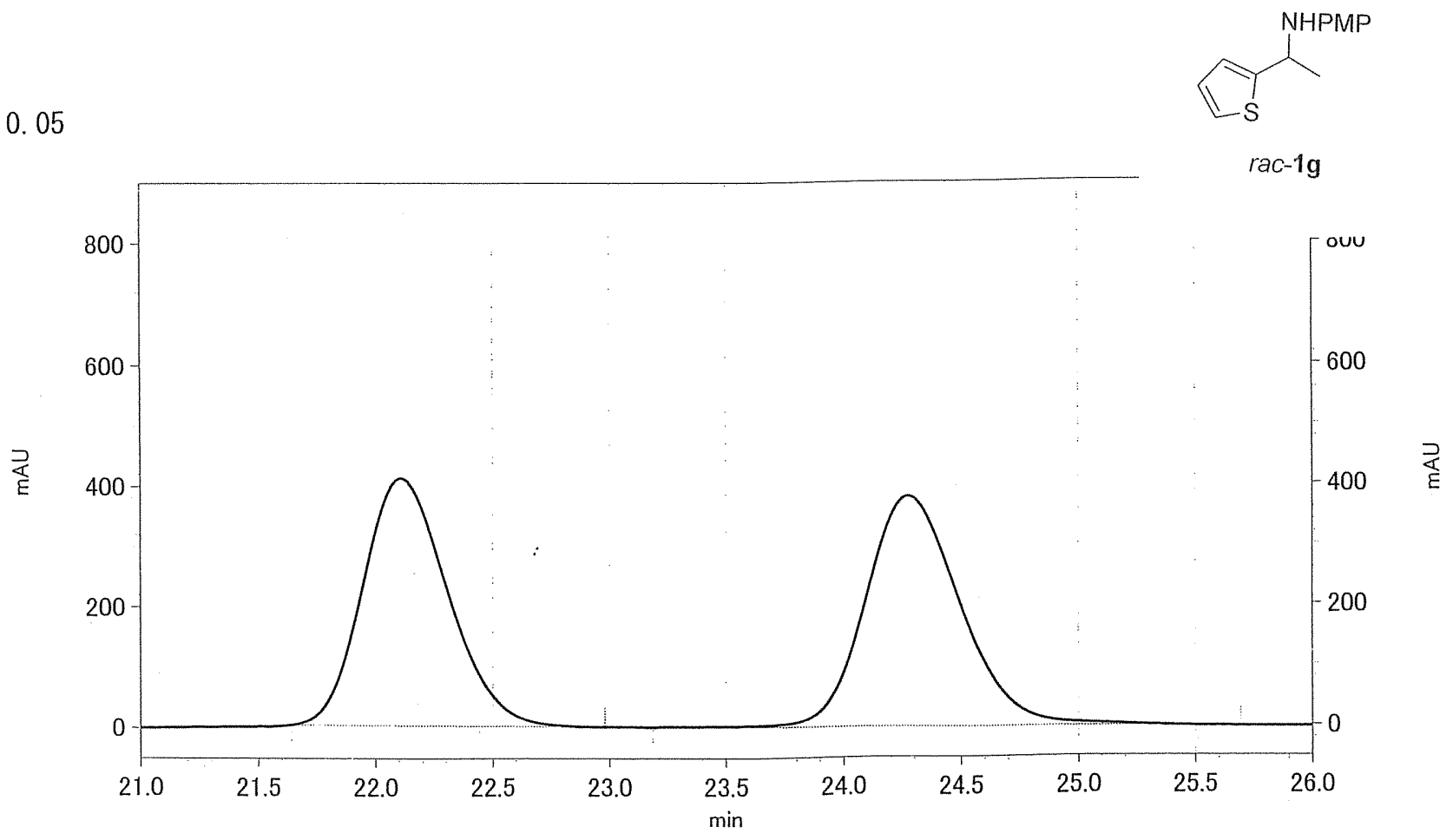

\begin{tabular}{ccccc} 
Peak \# & Retention time & Type & Area & Area \% \\
\hline 1 & 22.11 & $B B$ & 41207699 & 49.034 \\
2 & 24.27 & $B B$ & 42831880 & 50.966
\end{tabular}




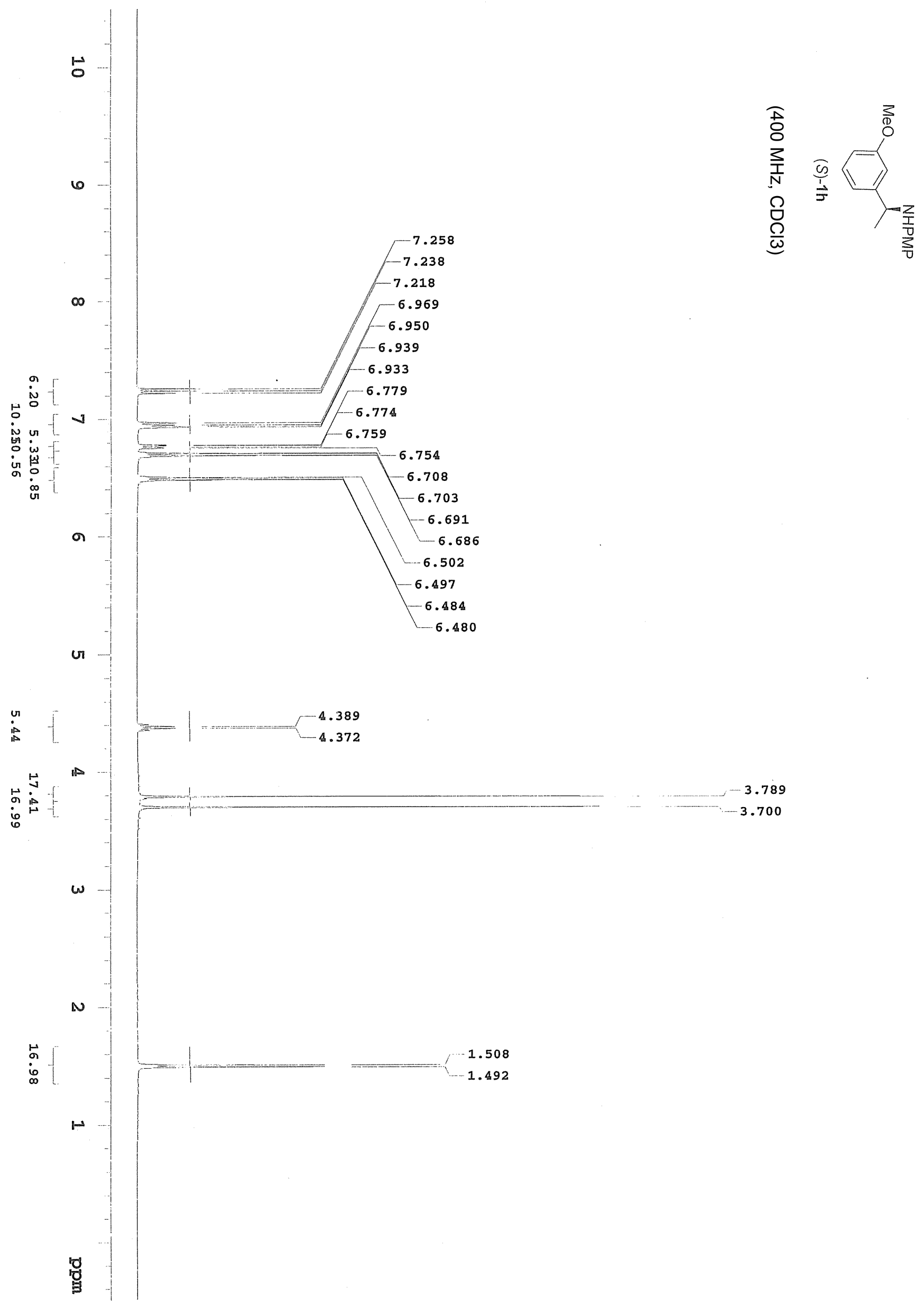




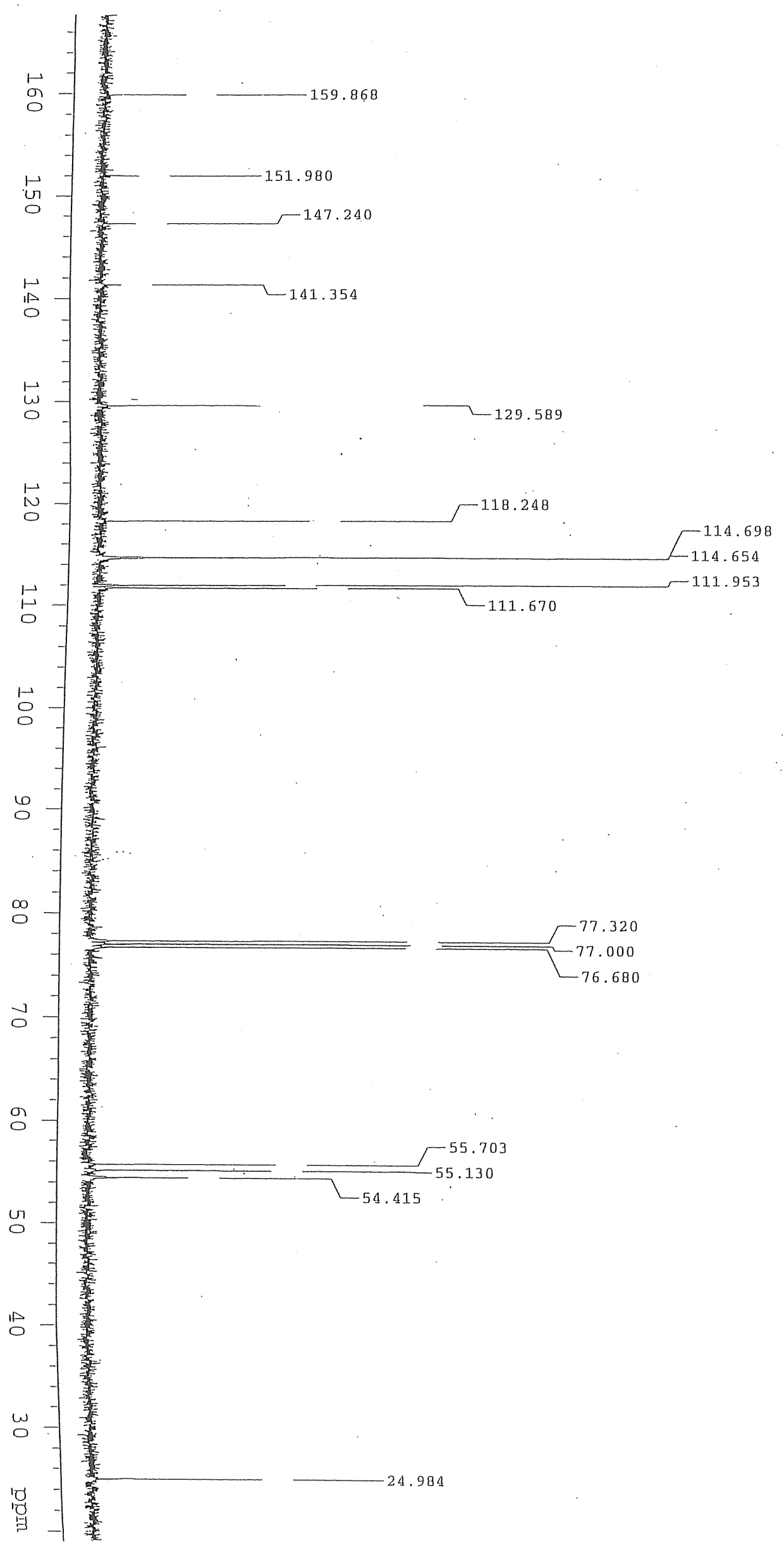


0.05

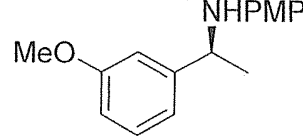

(S)-1h

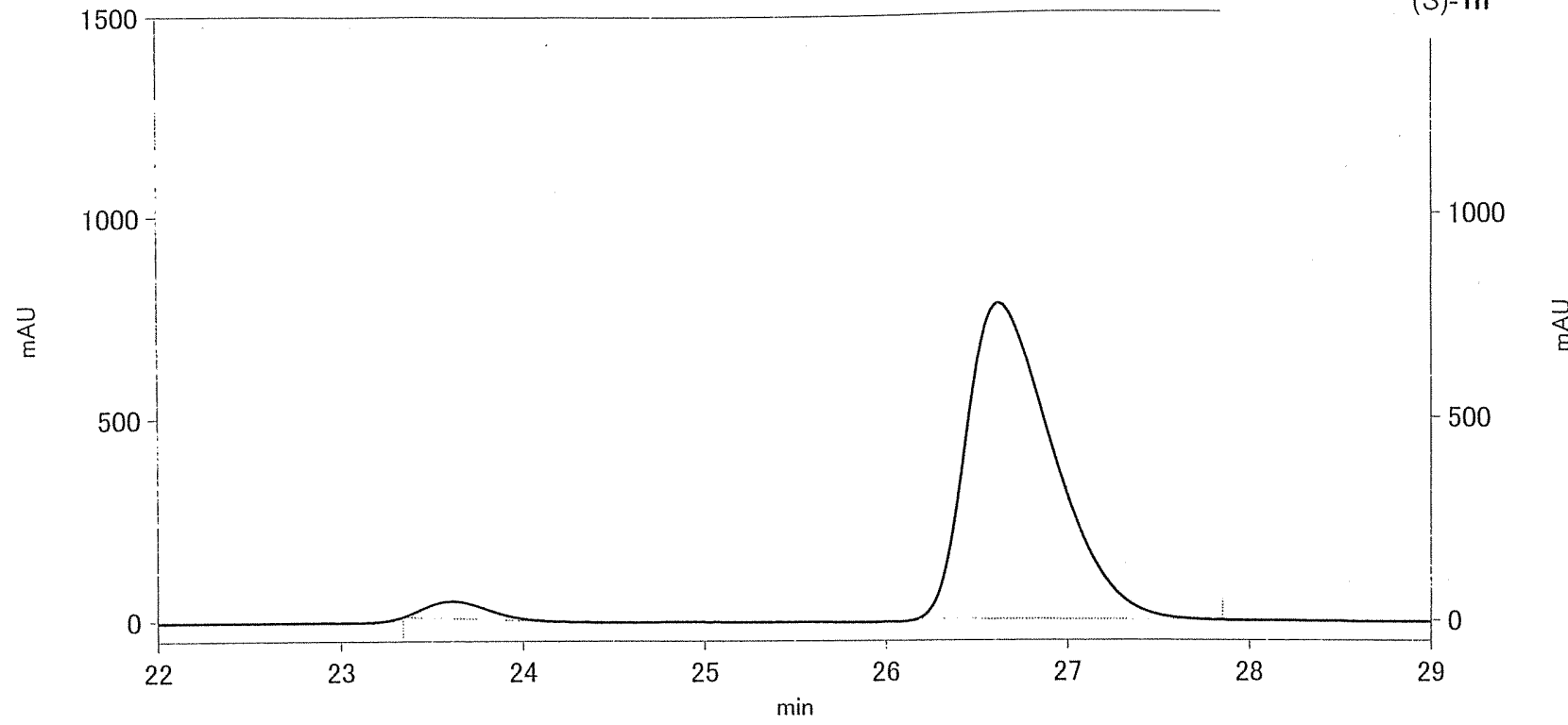

\begin{tabular}{ccccc} 
Peak \# & Retention time & Type & Area & Area \% \\
\hline 1 & 23.61 & $B B$ & 3923609 & 3.666 \\
2 & 26.62 & $B B$ & 103090505 & 96.334
\end{tabular}

0.05
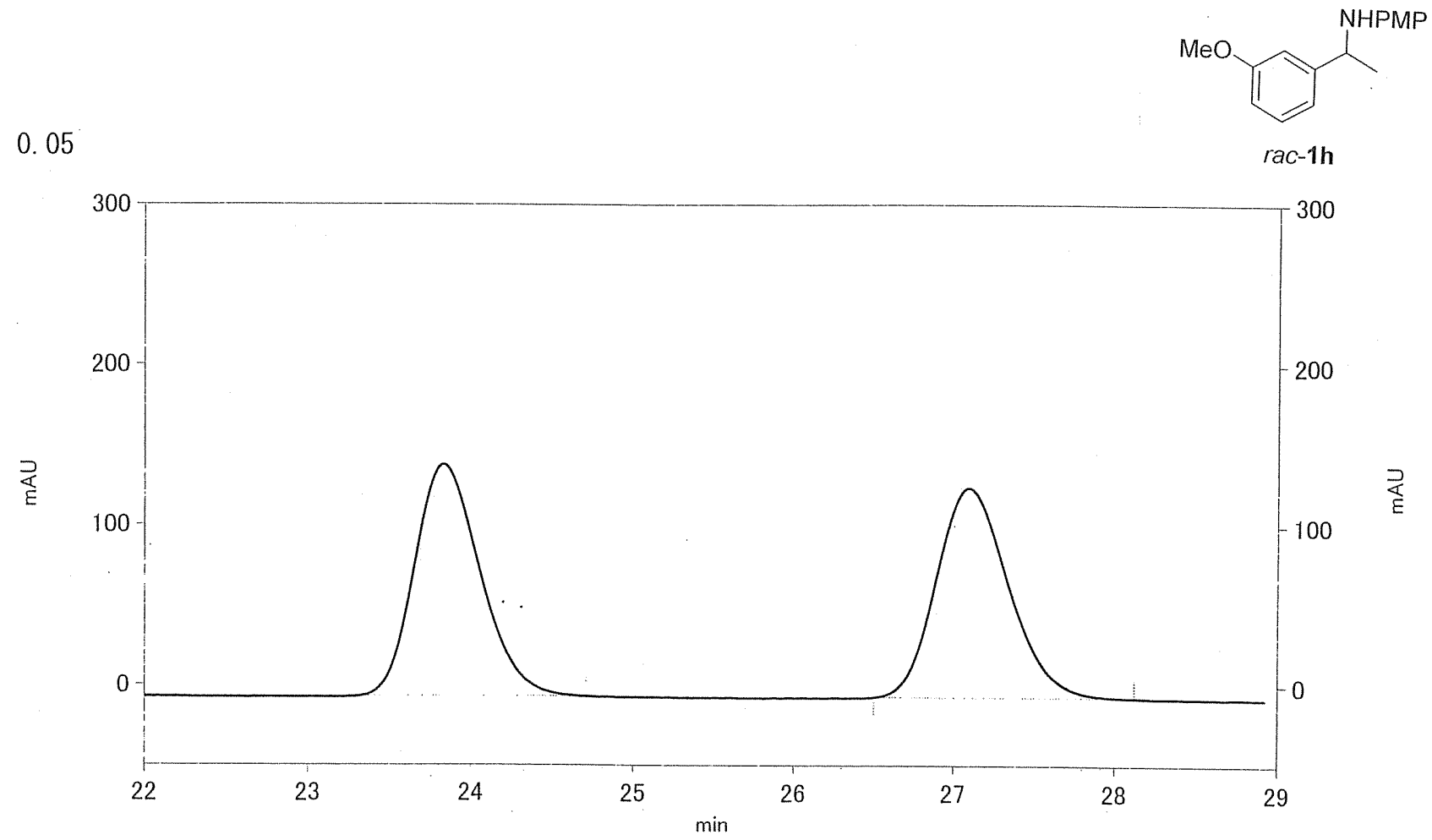

\begin{tabular}{ccccc} 
Peak \# & Retention time & Type & Area & Area \% \\
\hline 1 & 23.83 & $\mathrm{BB}$ & 16206434 & 49.721 \\
2 & 27.09 & $\mathrm{BB}$ & 16388404 & 50.279
\end{tabular}



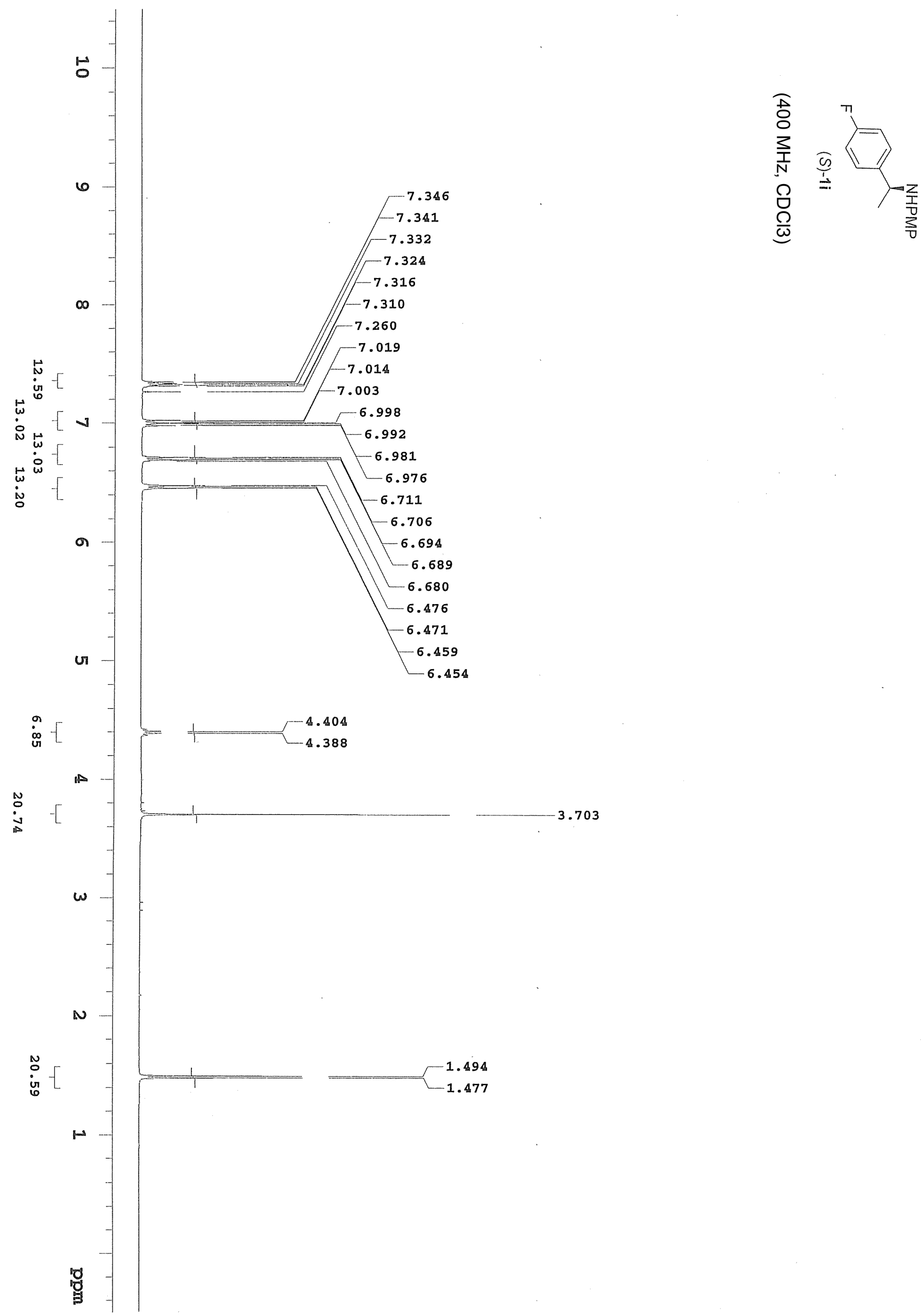


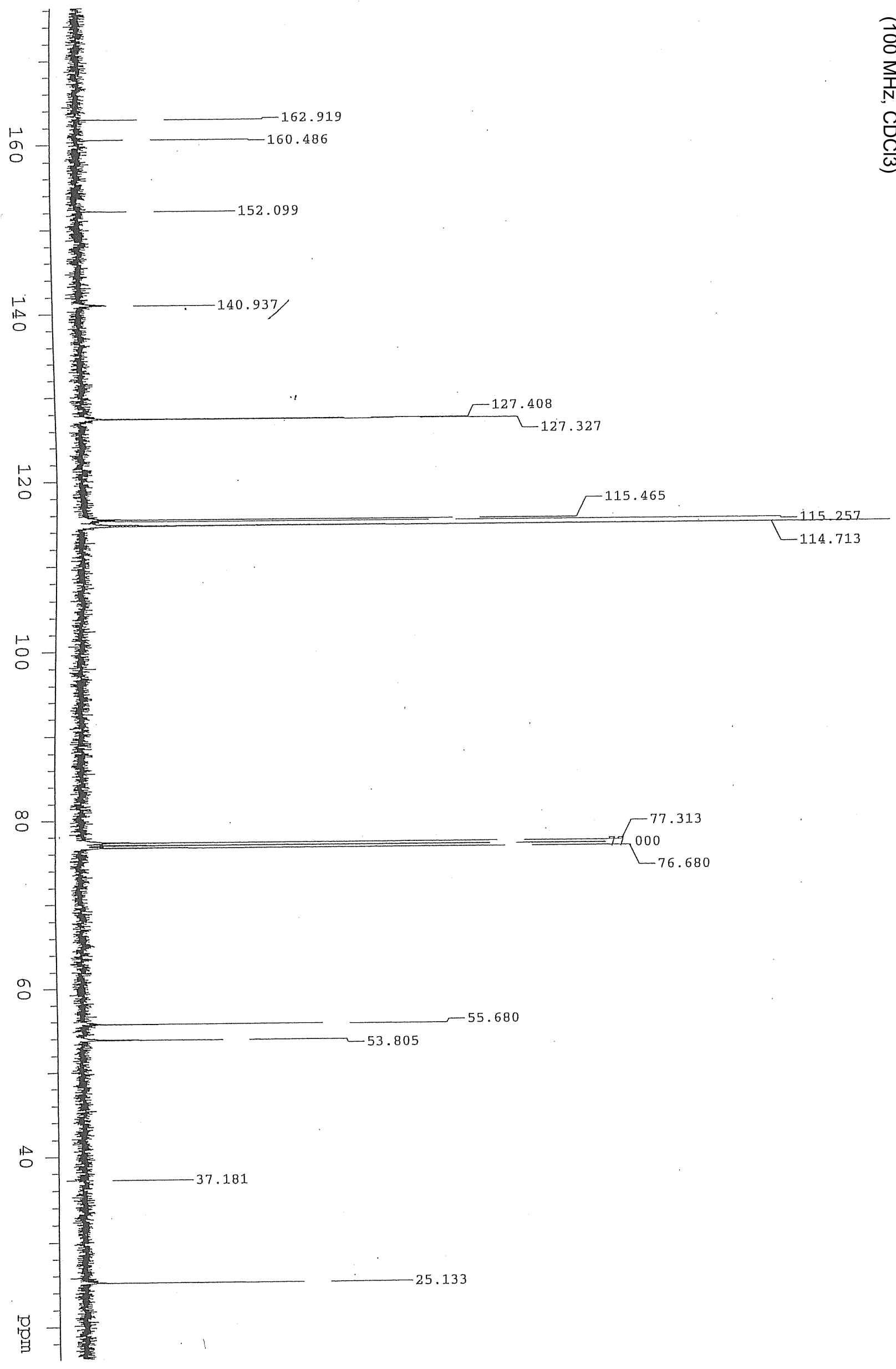

宫 


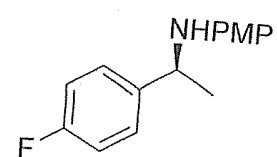

(S)-1i

0.05

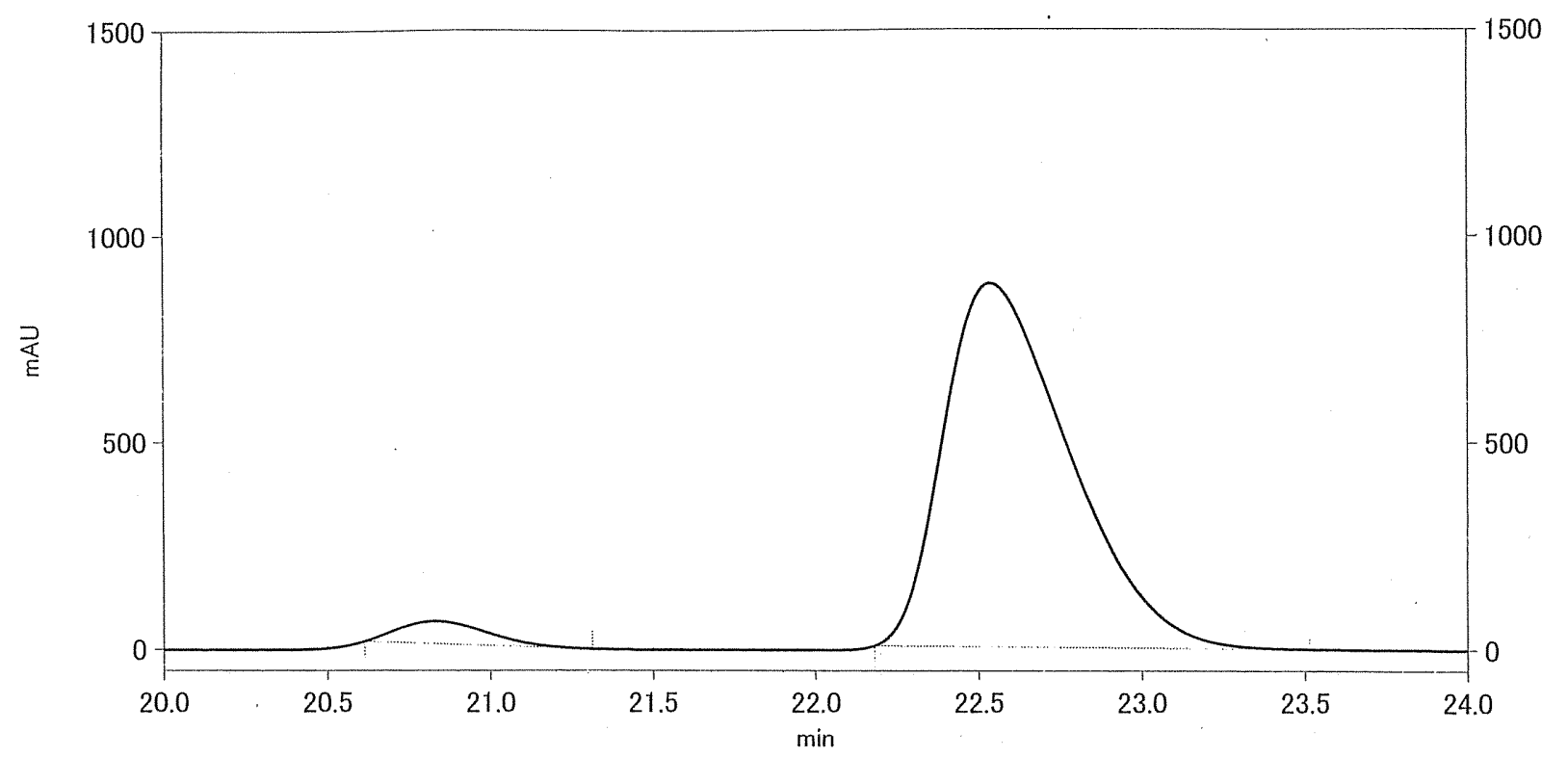

\begin{tabular}{ccccc} 
Peak \# & Retention time & Type & Area & Area \% \\
\hline 1 & 20.83 & BB & 4096876 & 4.193 \\
2 & 22.54 & BB & 93610098 & 95.807
\end{tabular}

0.05
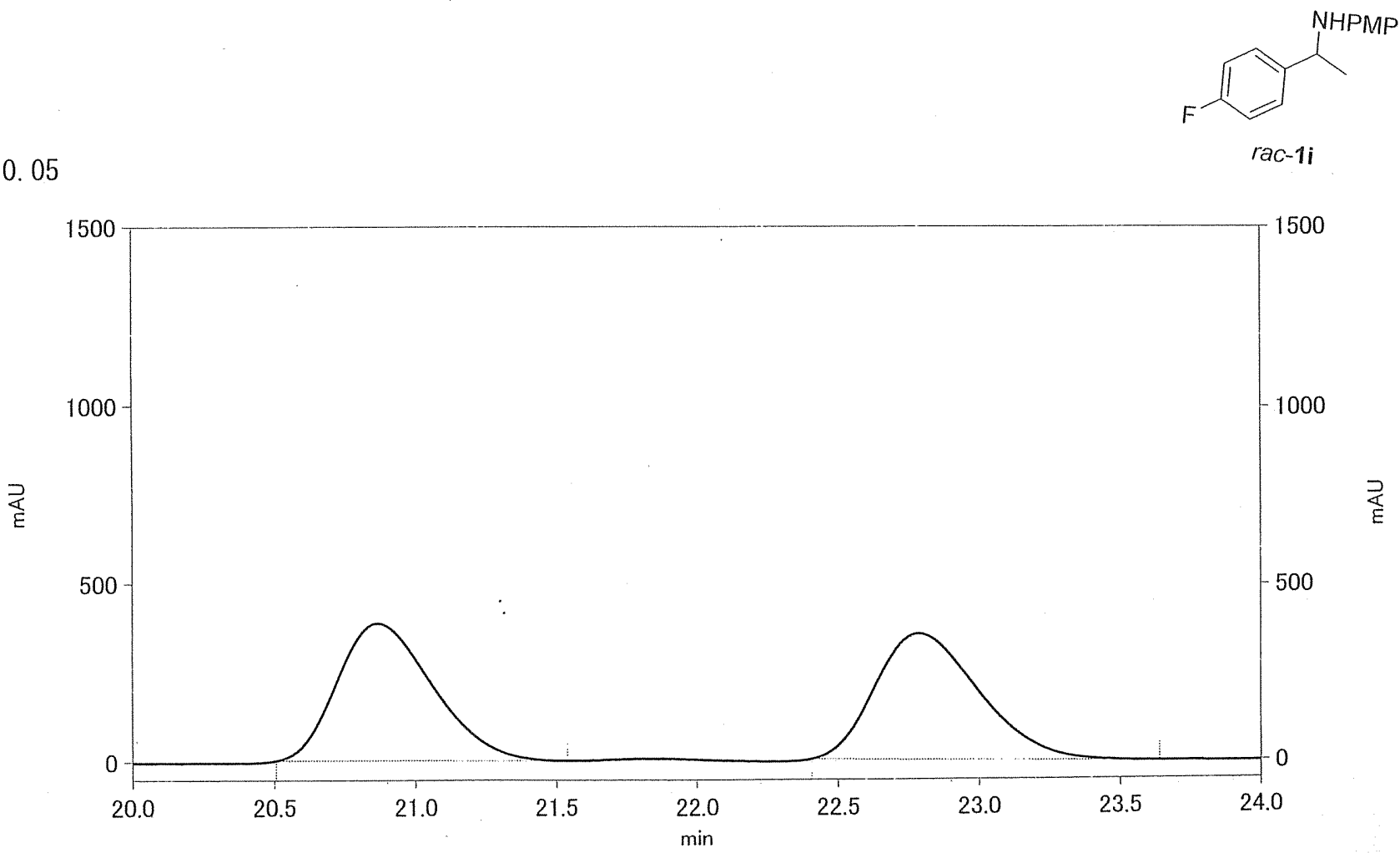

\begin{tabular}{ccccc} 
Peak \# & Retention time & Type & Area & Area \% \\
\hline 1 & 20.87 & BB & 36207627 & 49.883 \\
2 & 22.79 & BB & 36377067 & 50.117
\end{tabular}




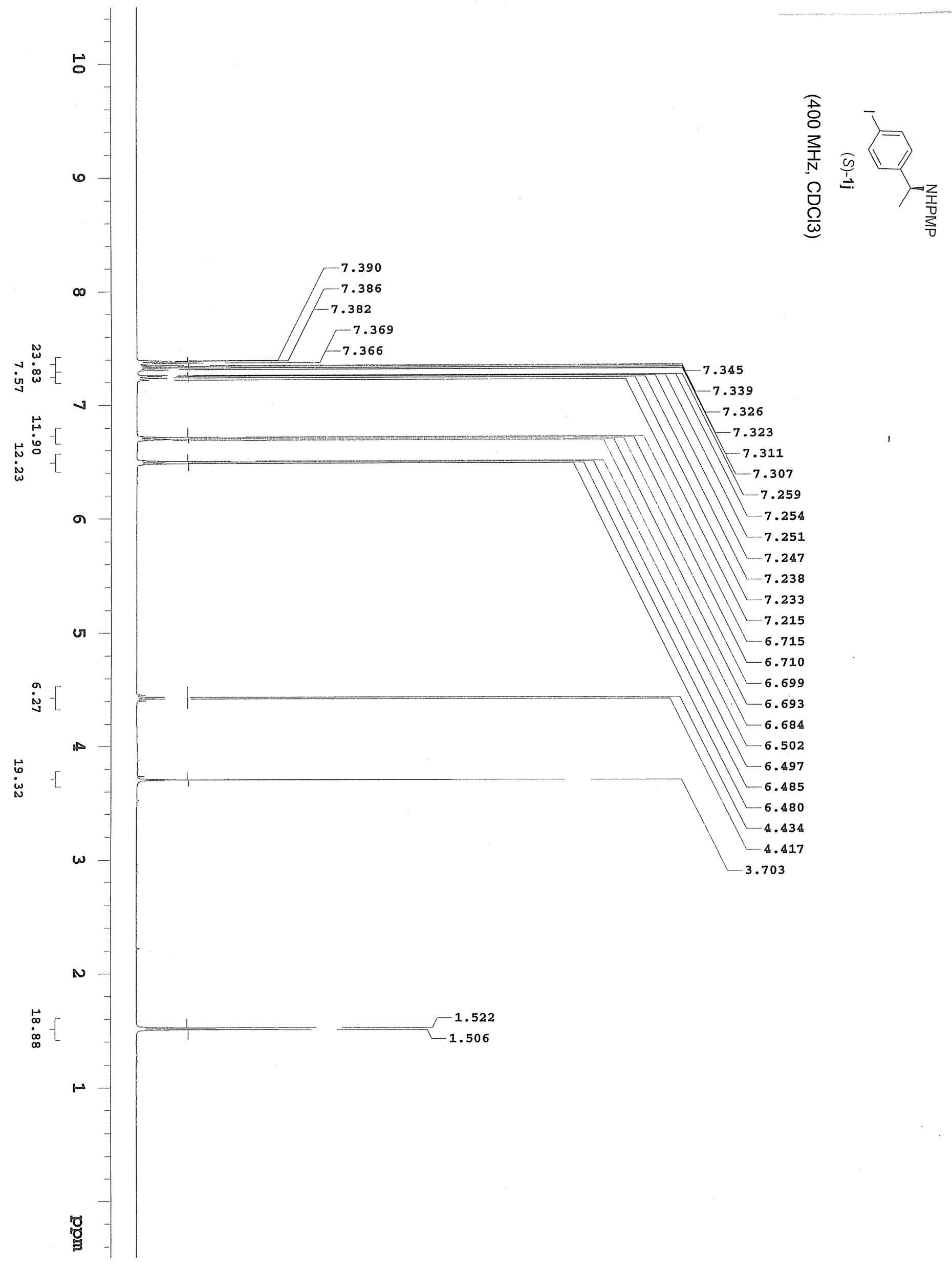




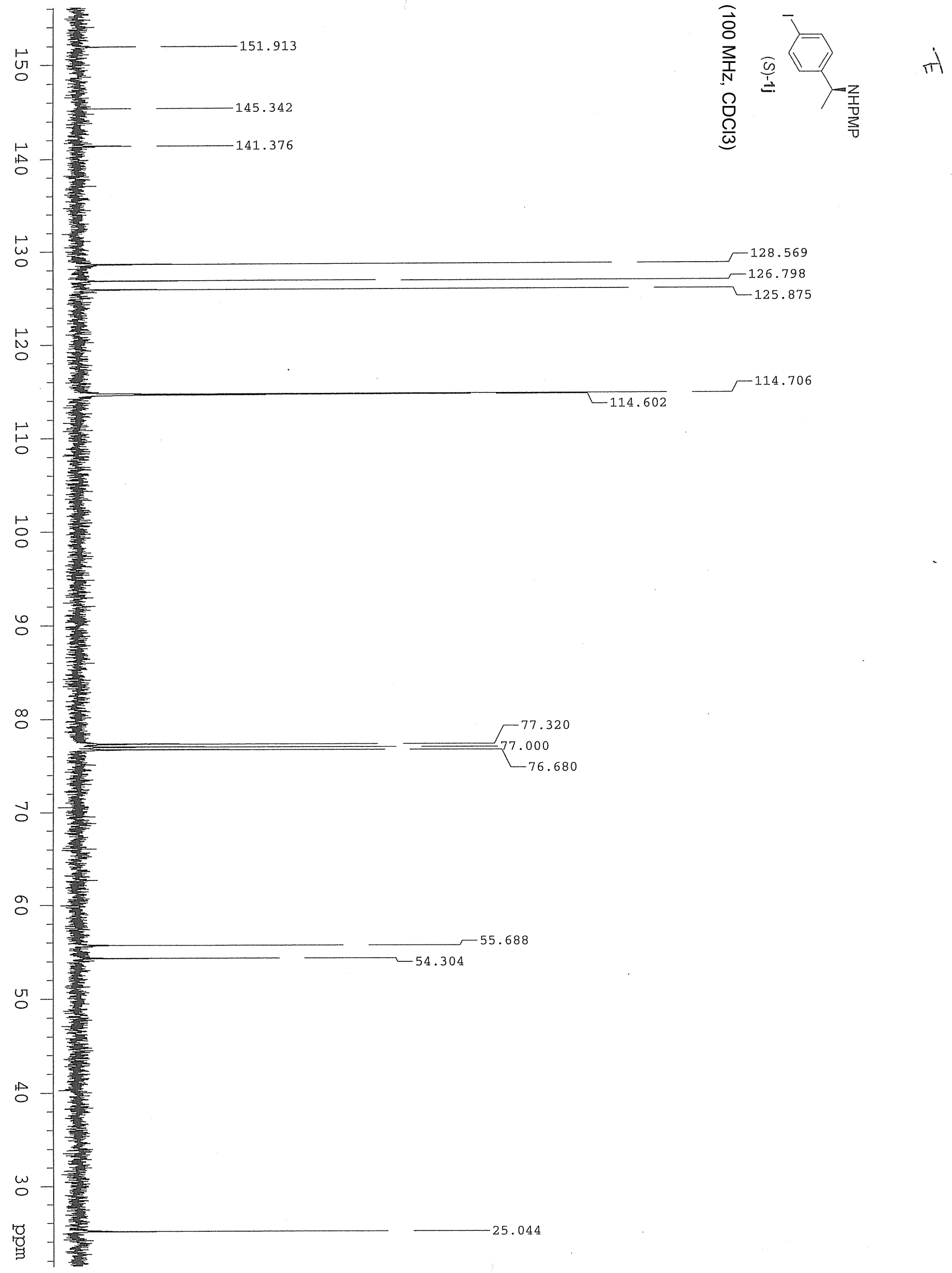


0.05

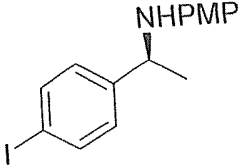

(S)-1j

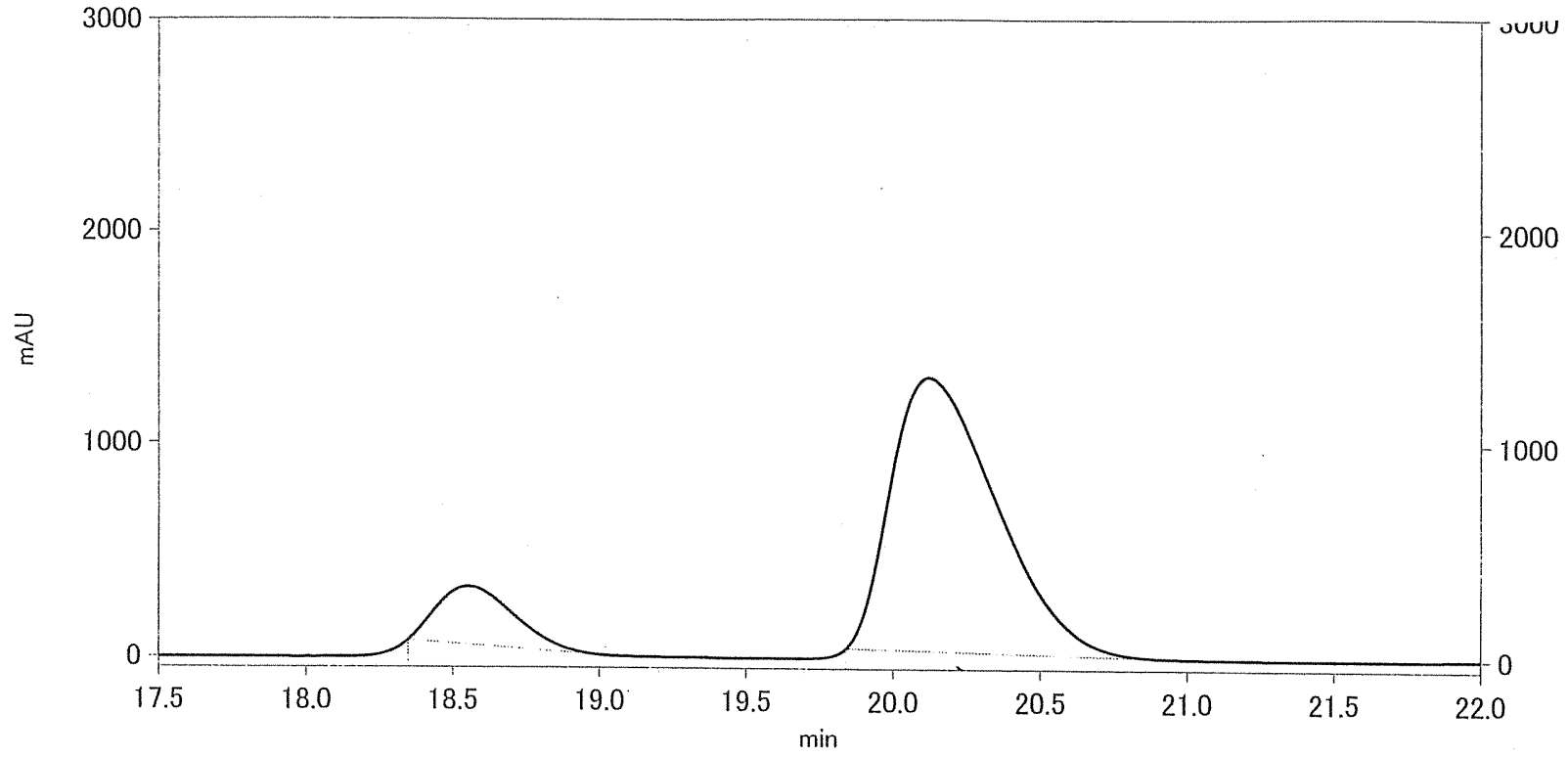

ᄅ্

\begin{tabular}{ccccc} 
Peak \# & Retention time & Type & Area & Area \% \\
\hline 1 & 18.55 & $B B$ & 19842870 & 13.474 \\
2 & 20.12 & $B B$ & 127420343 & 86.526
\end{tabular}

0.05

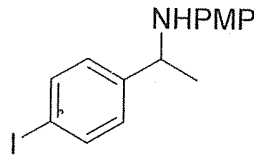

rac-1j

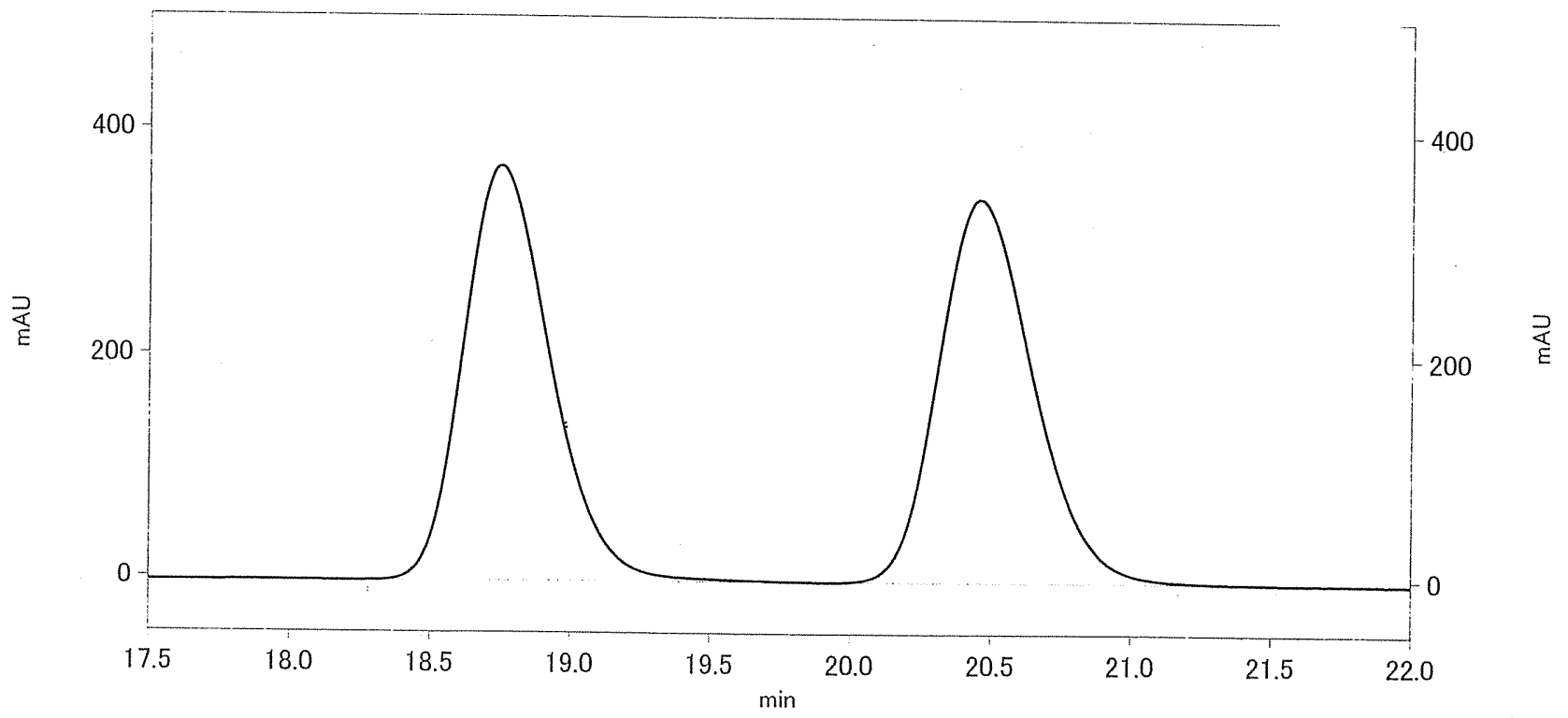

Peak \# Retention time

$\begin{array}{ll}1 & 18.75 \\ 2 & 20.46\end{array}$

Type

Area 


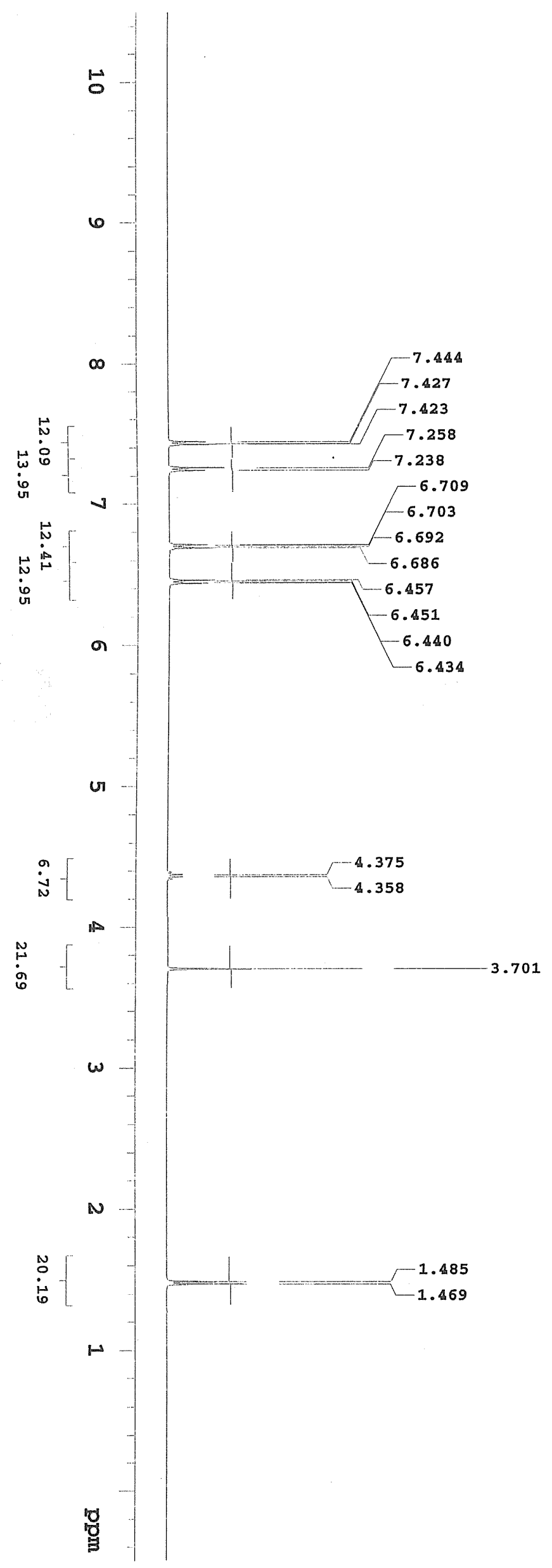




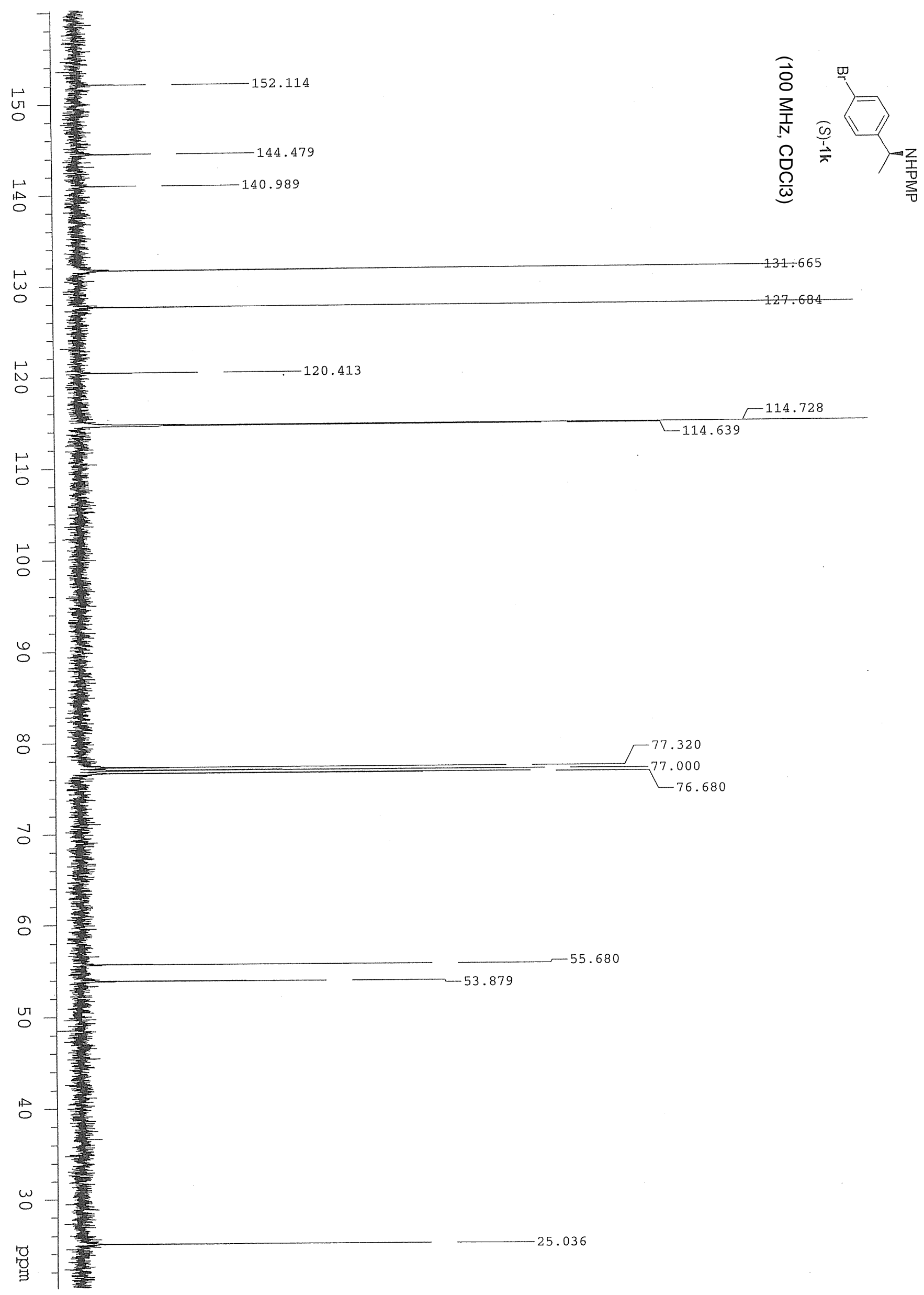




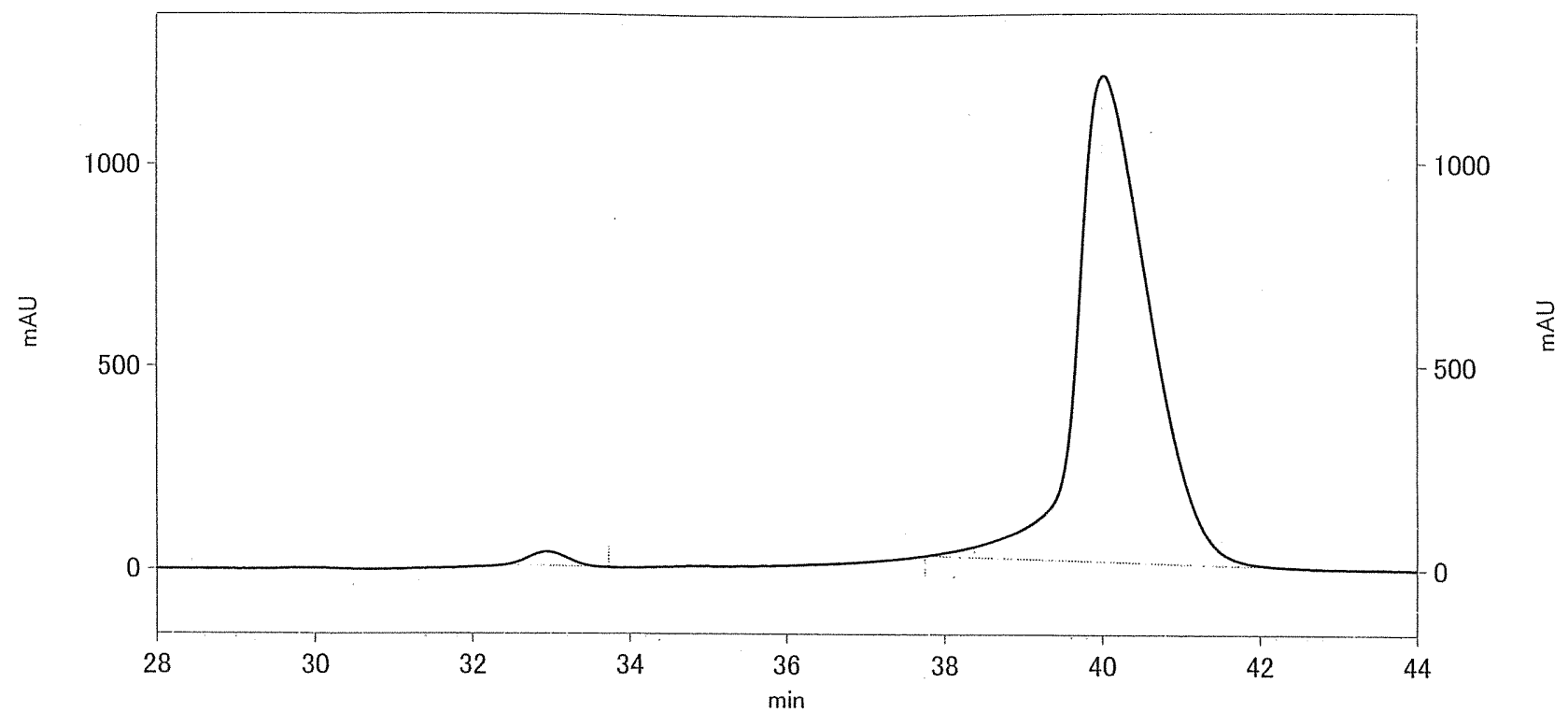

\begin{tabular}{ccccc} 
Peak \# & Retention time & Type & Area & Area \% \\
\hline 1 & 32.95 & $\mathrm{BB}$ & 4816310 & 1.537 \\
2 & 40.03 & $\mathrm{BB}$ & 308614856 & 98.463
\end{tabular}

0.05

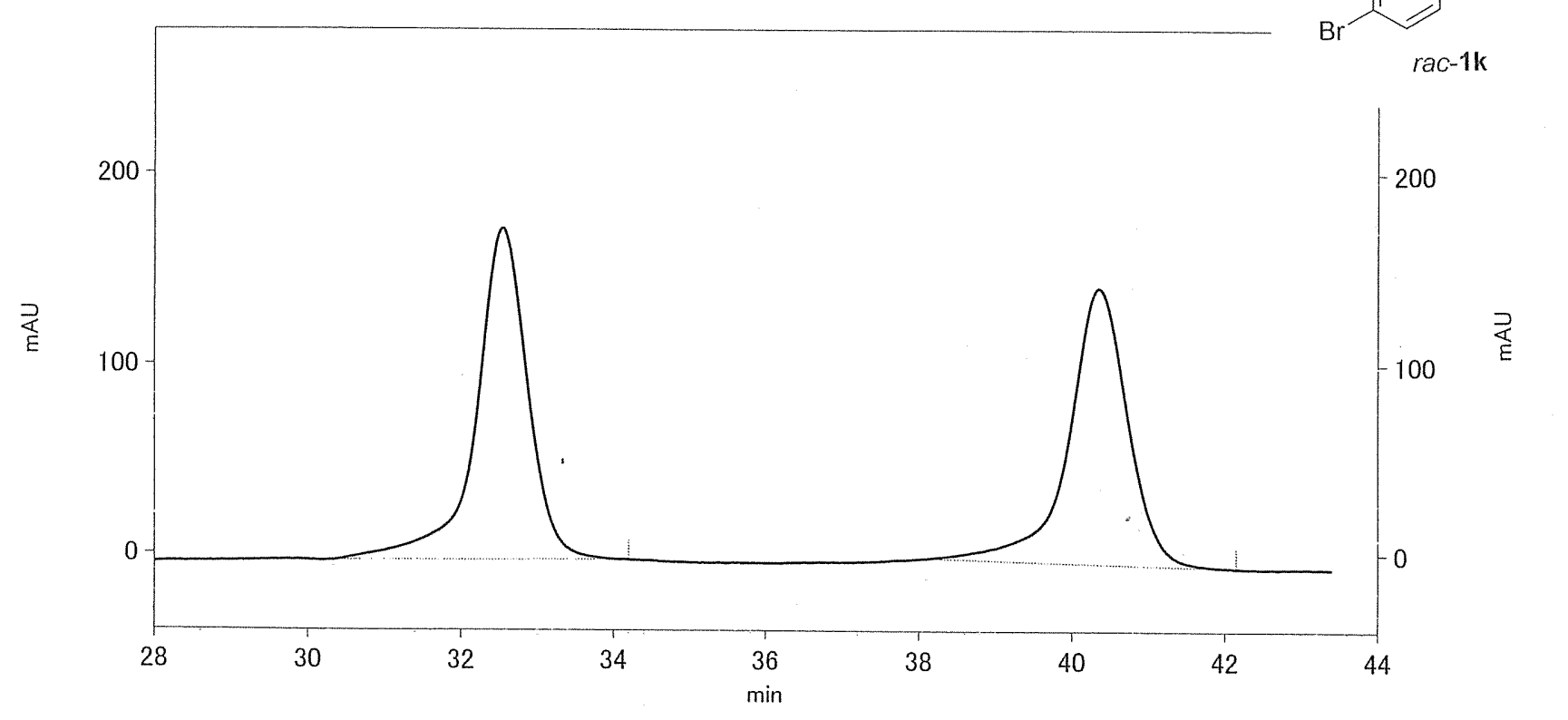

\begin{tabular}{ccccc} 
Peak \# & Retention time & Type & Area & Area \% \\
\hline 1 & 32.55 & $B B$ & 32949816 & 51.089 \\
2 & 40.34 & BB & 31545552 & 48.911
\end{tabular}




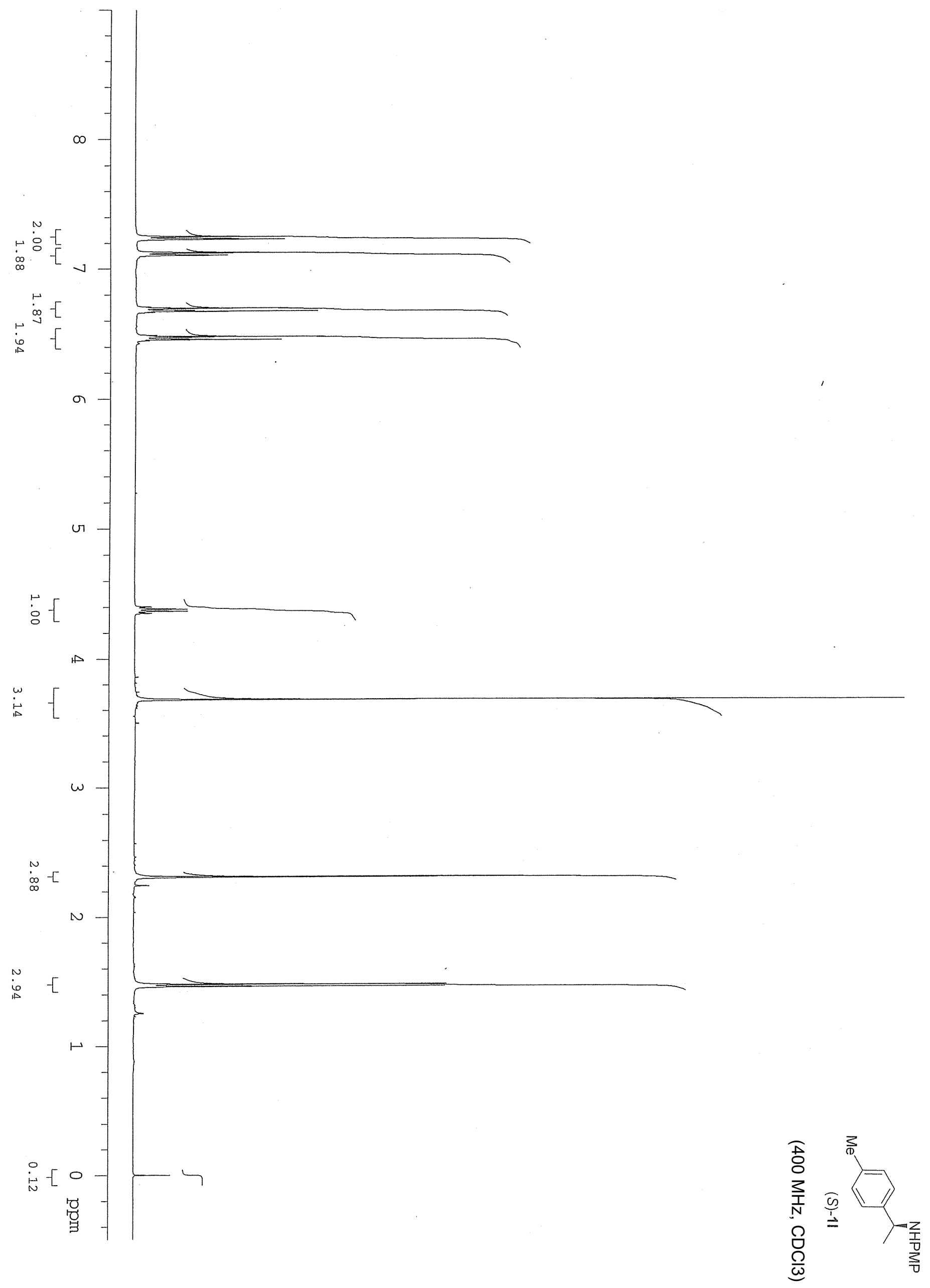




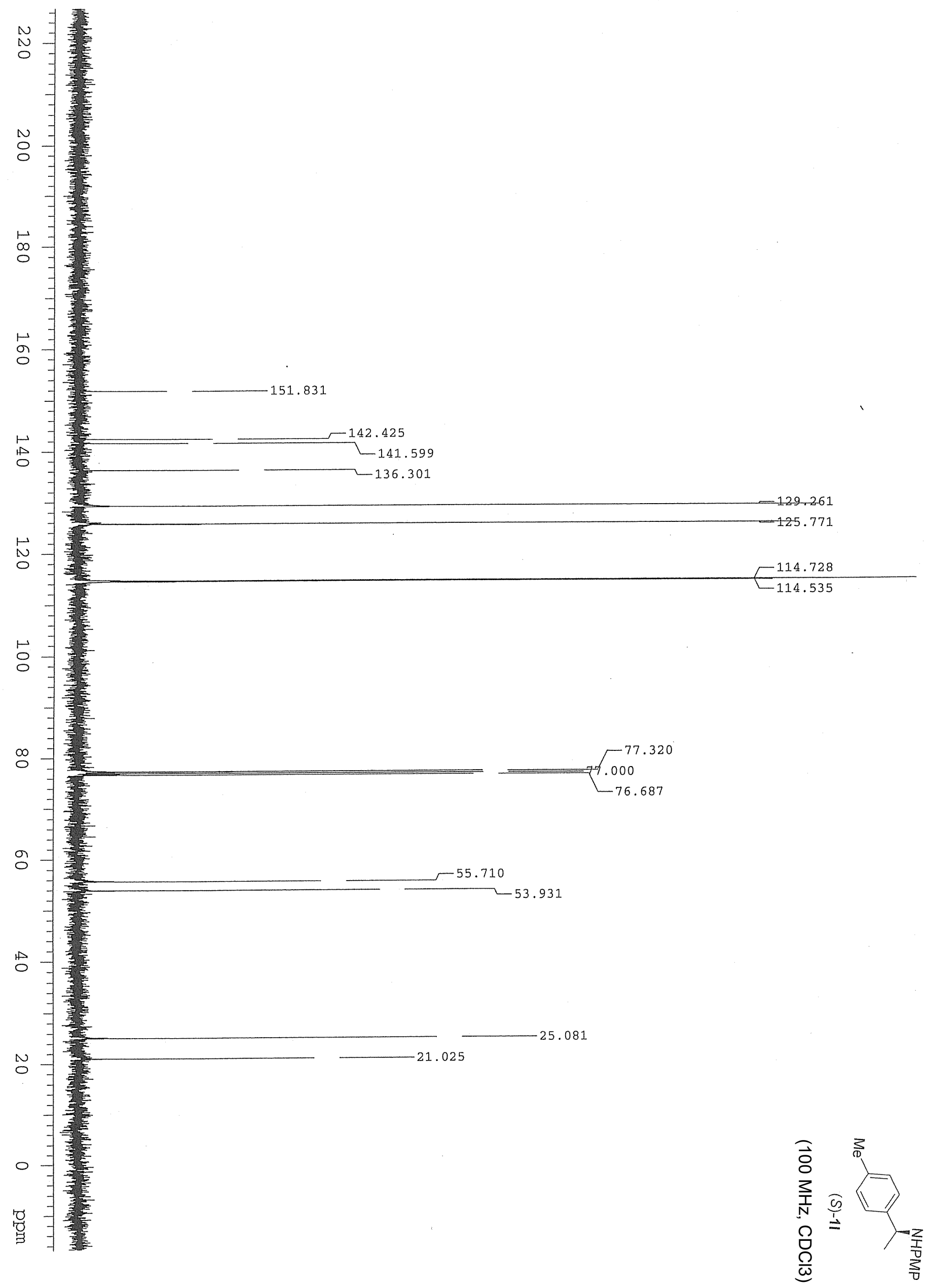


0.05

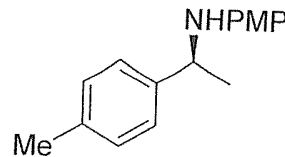

(S)-11

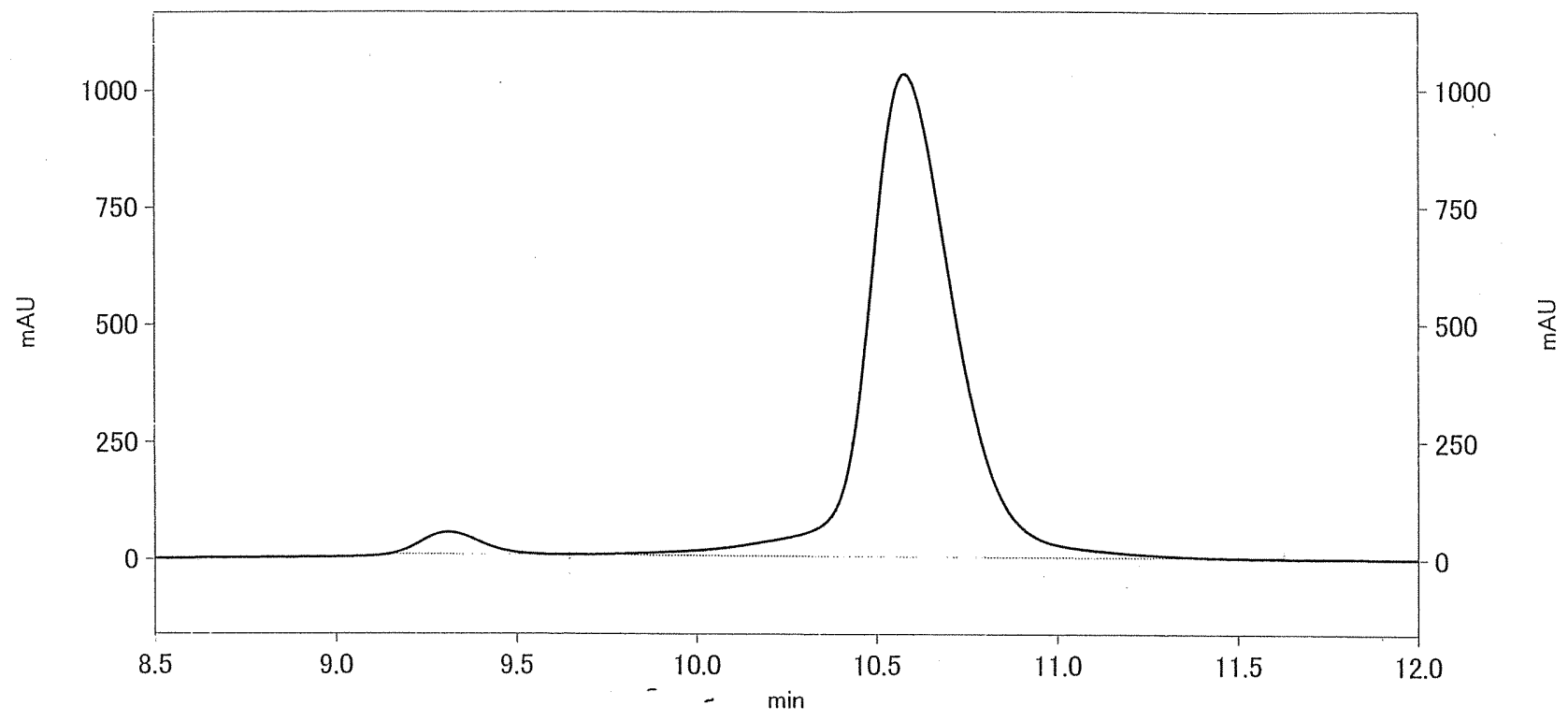

\begin{tabular}{|c|c|c|c|c|}
\hline Peak \# & Retention time & Type & Area & Area $\%$ \\
\hline $\begin{array}{l}1 \\
2\end{array}$ & $\begin{array}{c}9.31 \\
10.58\end{array}$ & $\begin{array}{l}\text { BV } \\
\text { VB }\end{array}$ & $\begin{array}{r}2200992 \\
69327707\end{array}$ & $\begin{array}{c}3.077 \\
96.923\end{array}$ \\
\hline
\end{tabular}

0.05

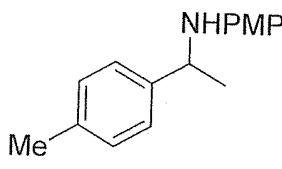

rac-11

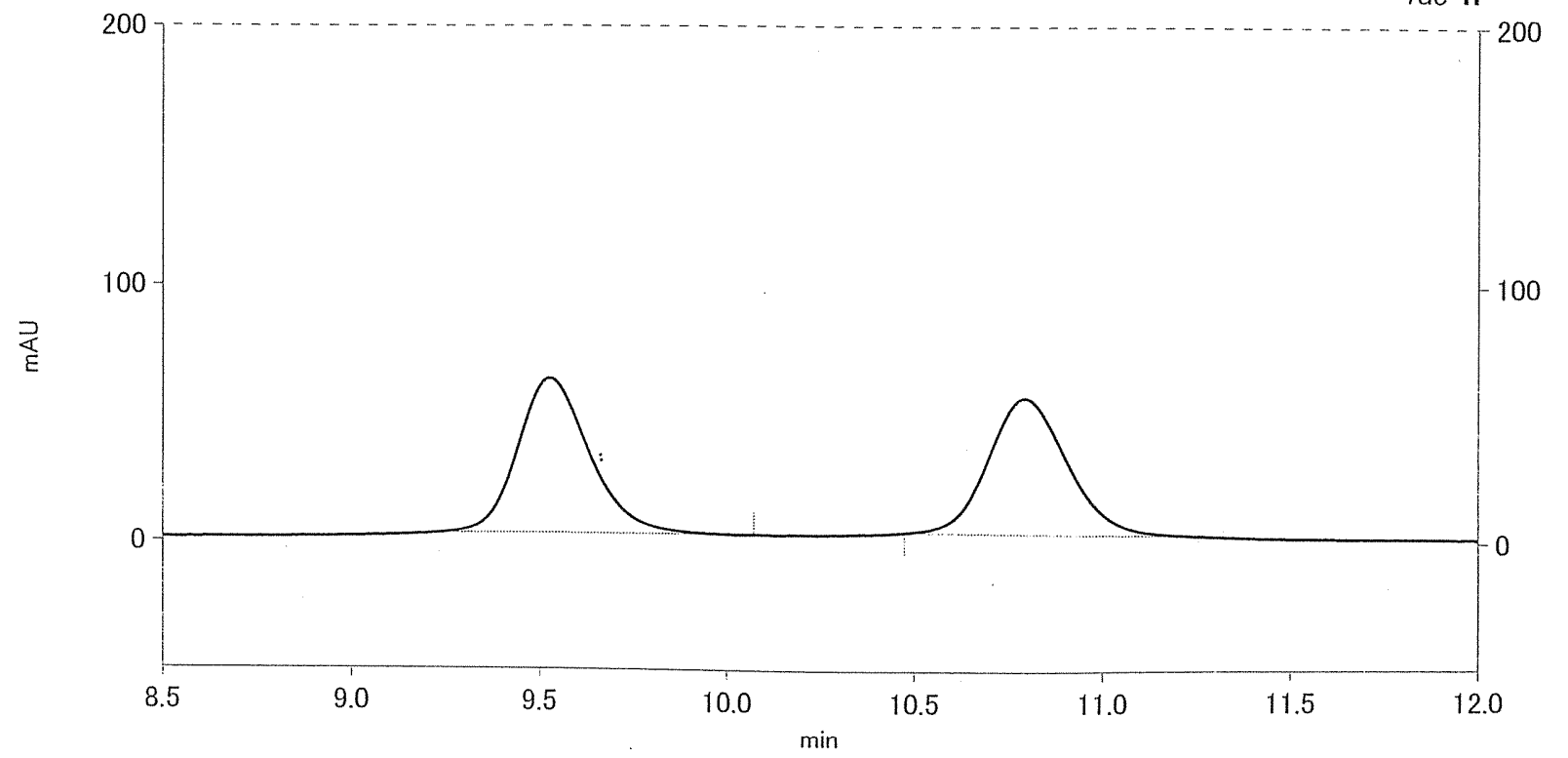

$\mathrm{BB}$ 


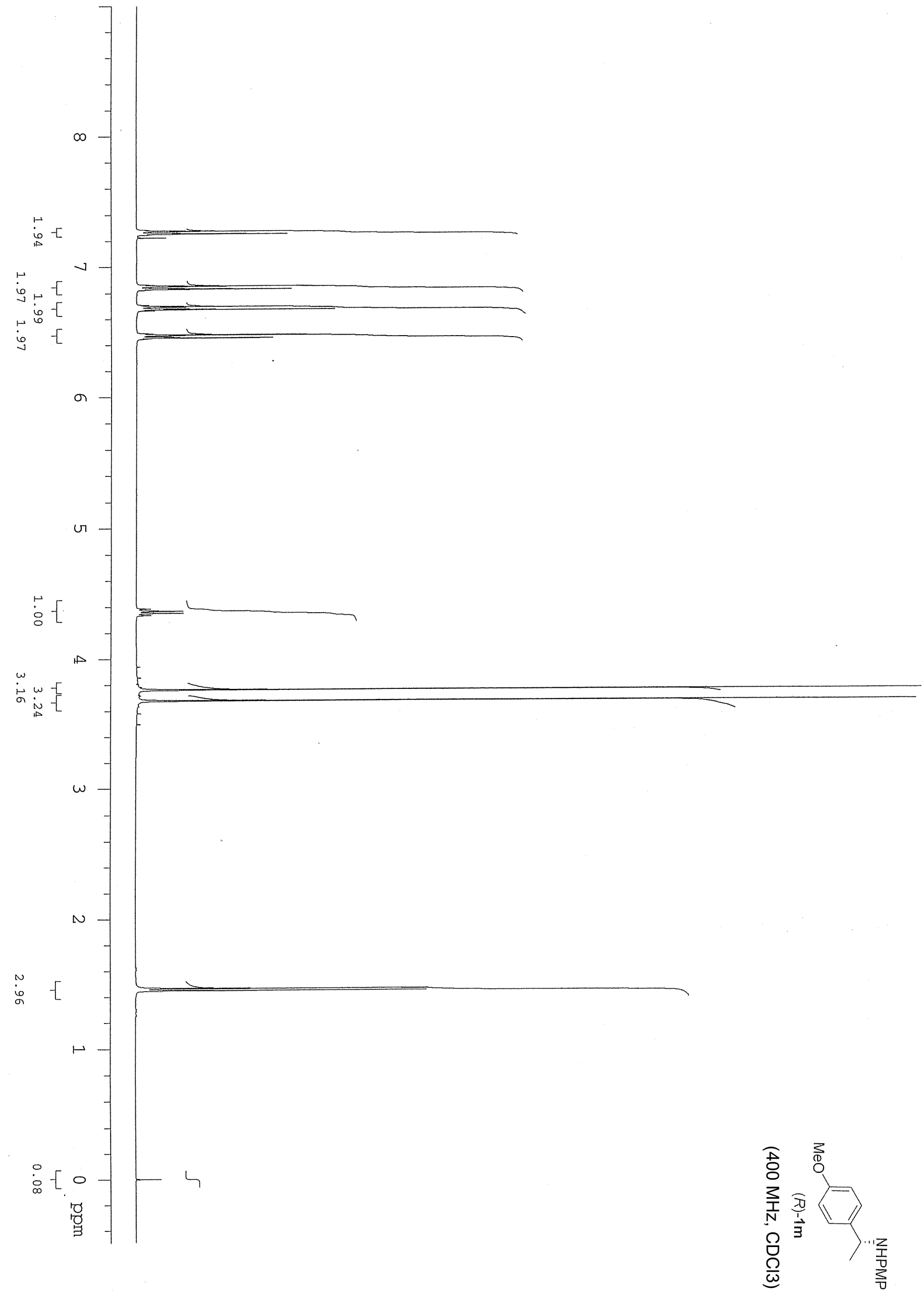




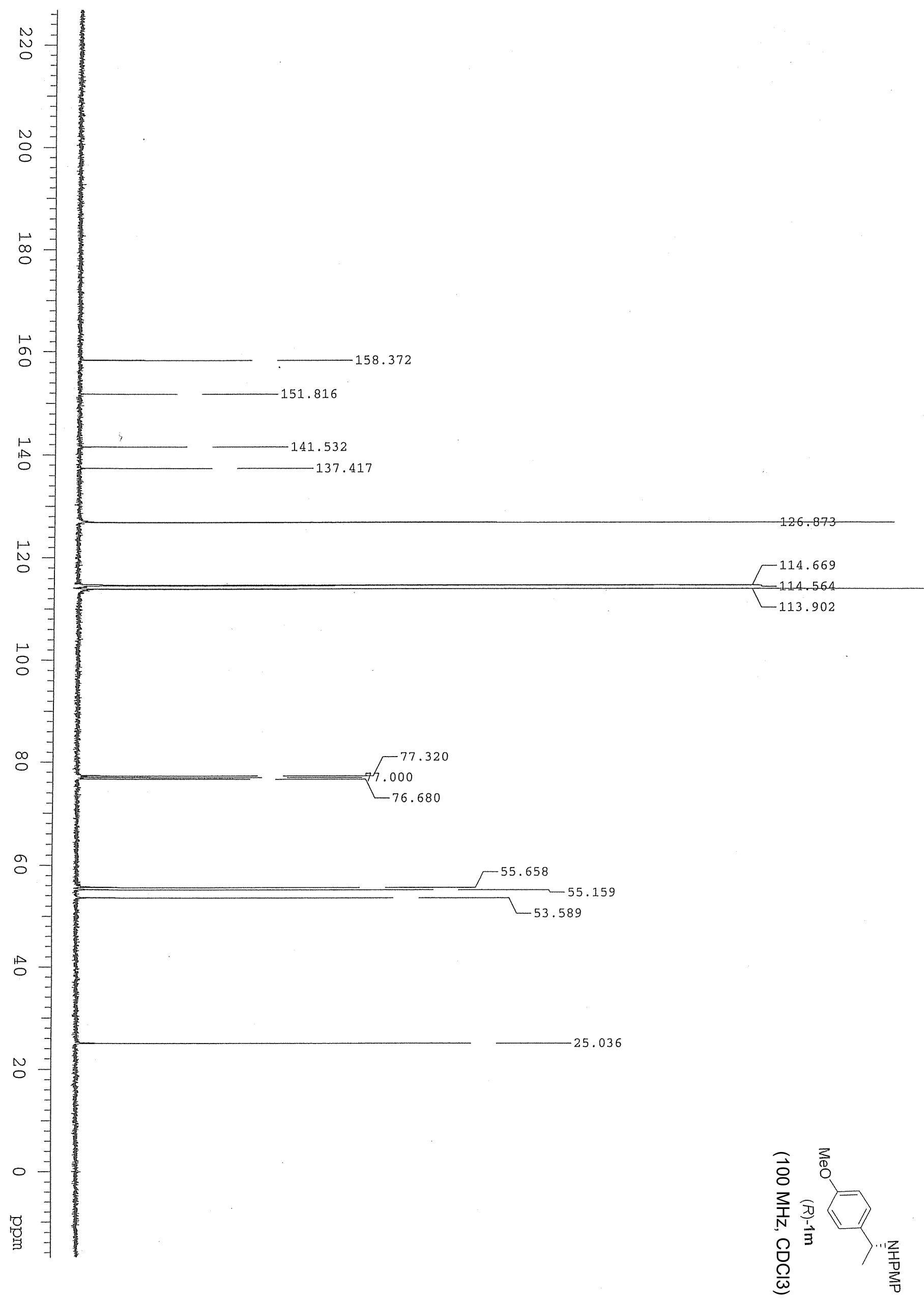


0.05

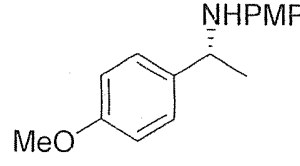

(R)-1m

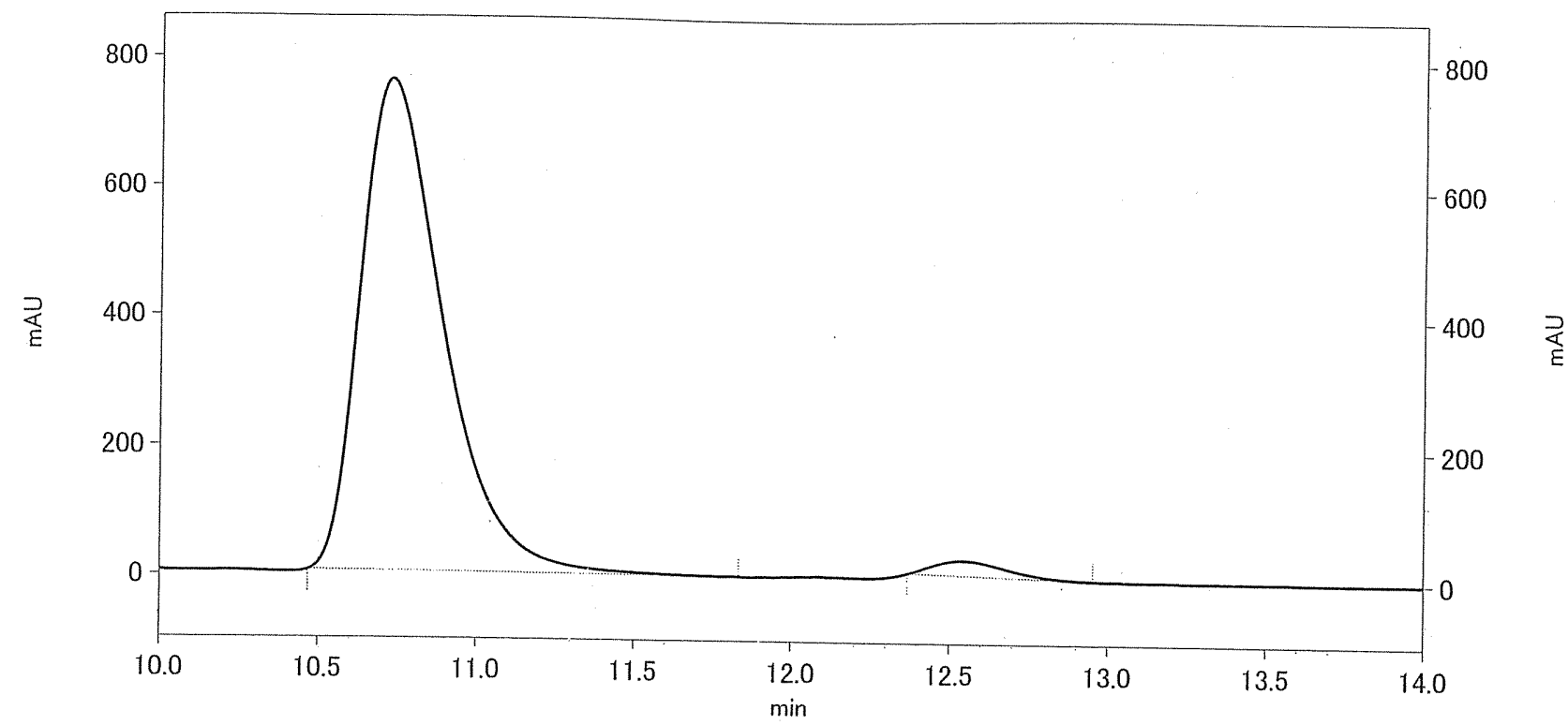

\begin{tabular}{ccccc} 
Peak \# & Retention time & Type & Area & Area \% \\
\hline 1 & 10.73 & $B B$ & 55406180 & 97.360 \\
2 & 12.54 & $B B$ & 1502234 & 2.640
\end{tabular}

0.05
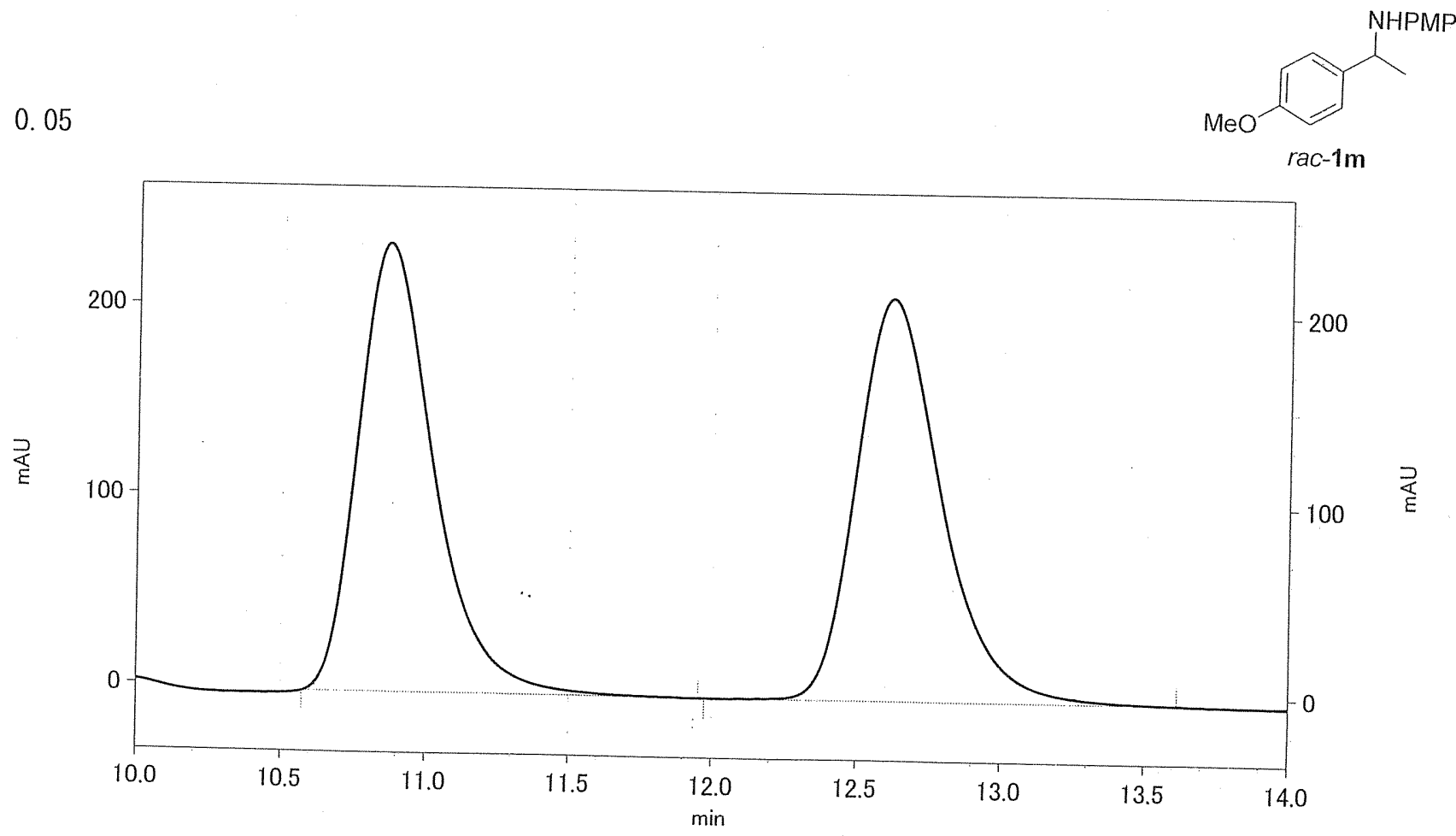

Peak \# Retention time

$1 \quad 10.87$

12. 61

Type 







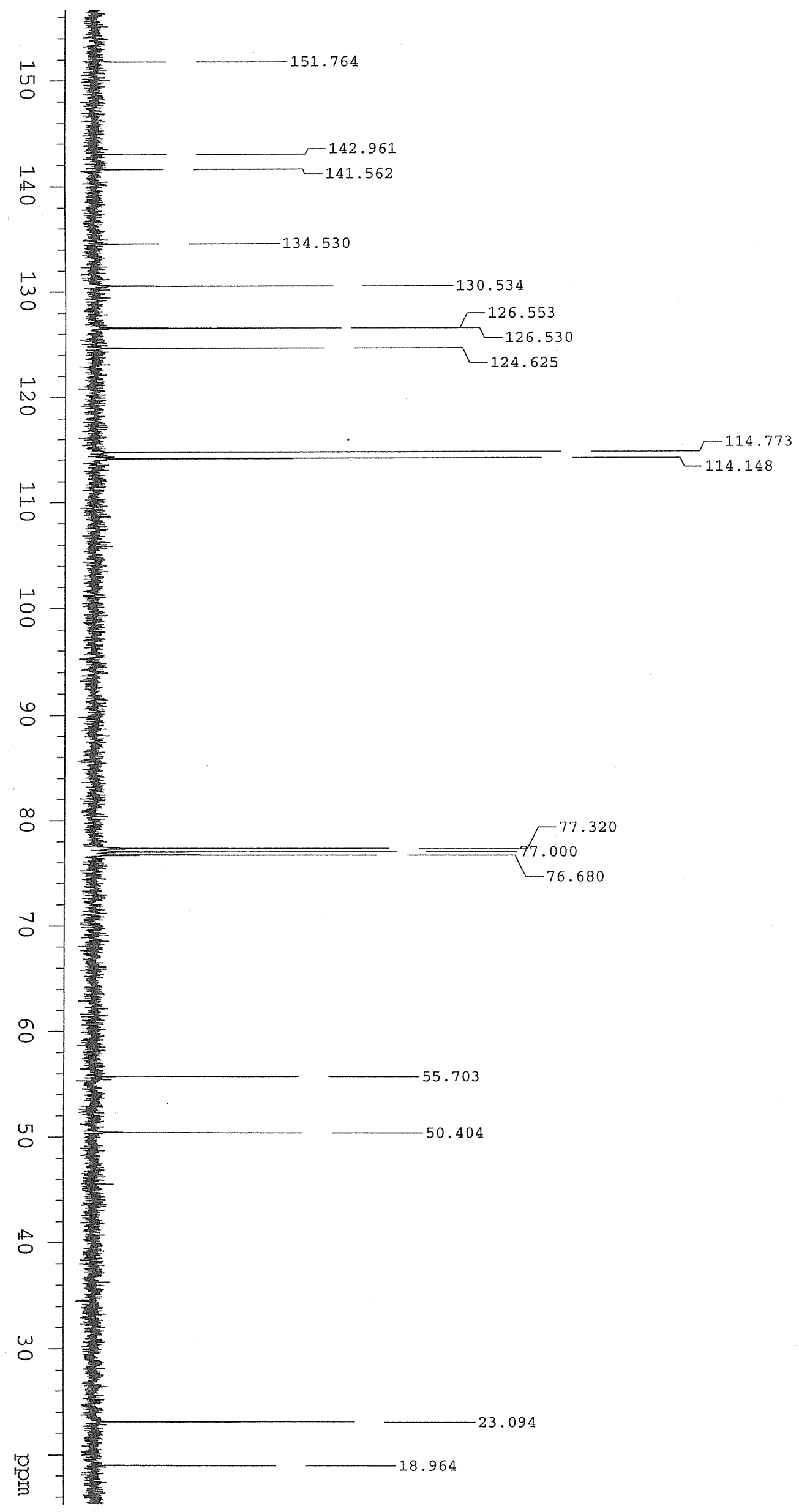




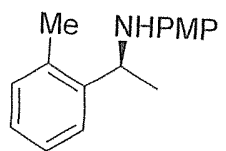

(S)- $\ln$

0.05

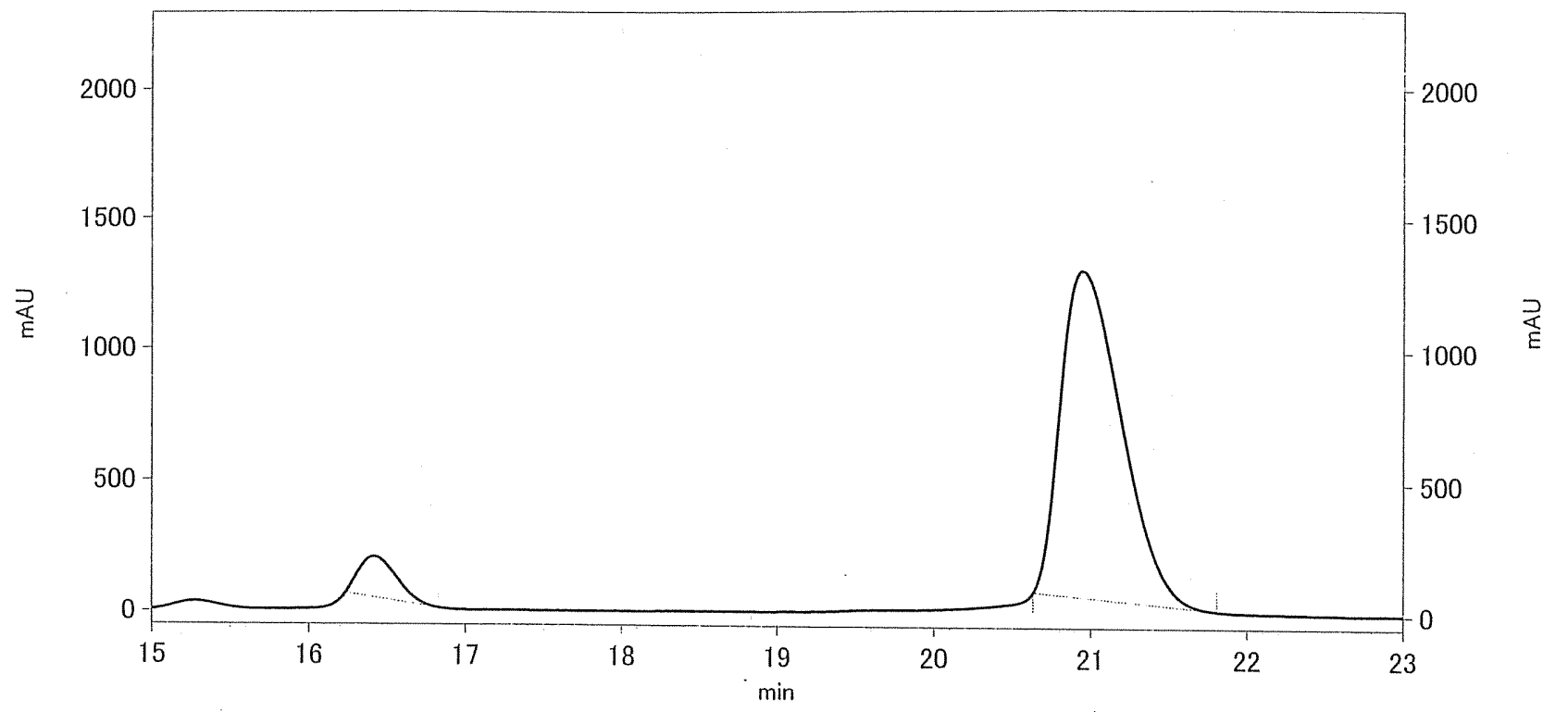

\begin{tabular}{ccccc} 
Peak \# & Retention time & Type & Area & Area \% \\
\hline 1 & 16.42 & $B B$ & 10406543 & 7.190 \\
2 & 20.95 & $B 1$ & 134321644 & 92.810
\end{tabular}

0.05
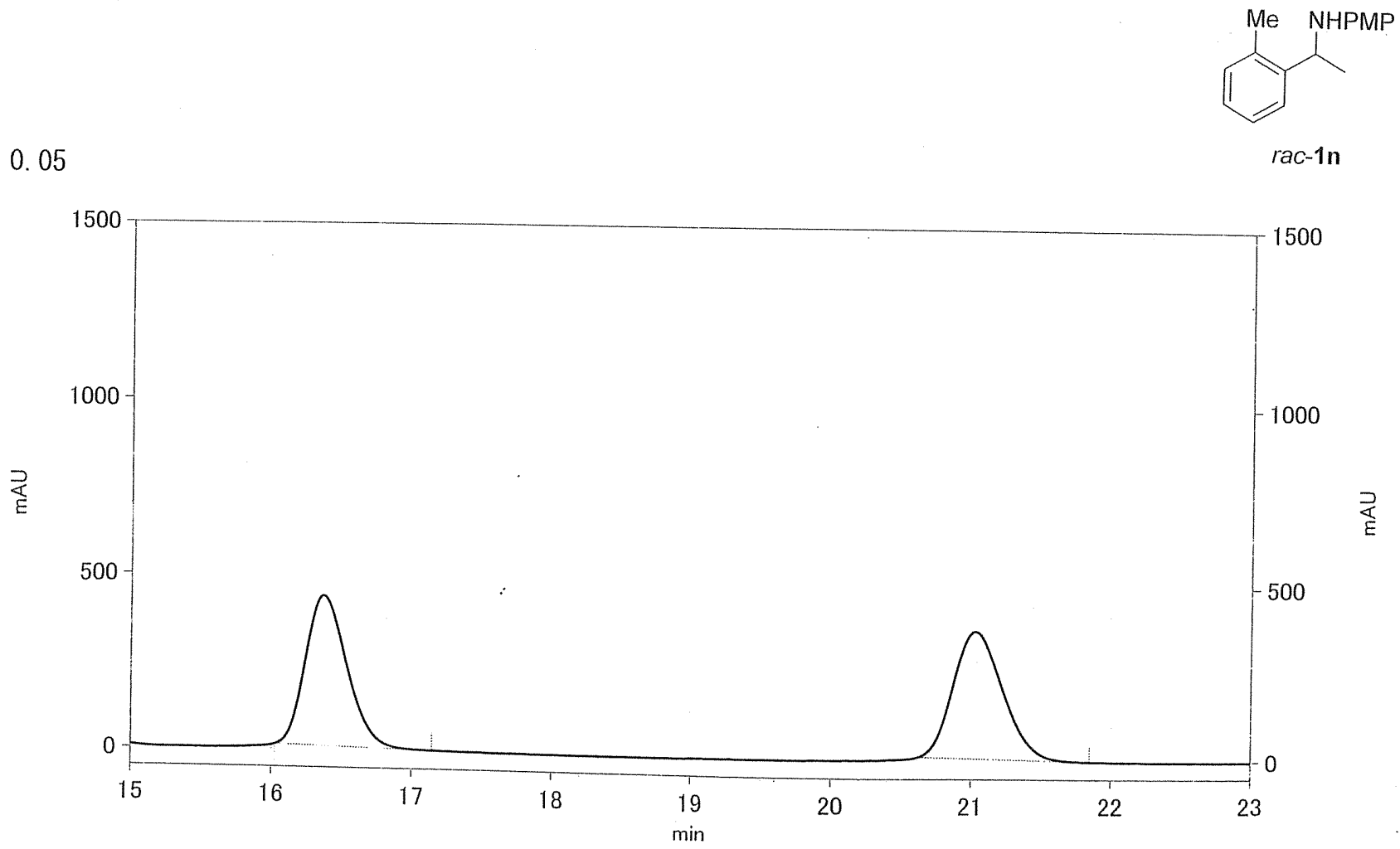

Peak \#

Retention time

16. 37

Type

Area

35715465

35868000

Area \%

BB

50.107 


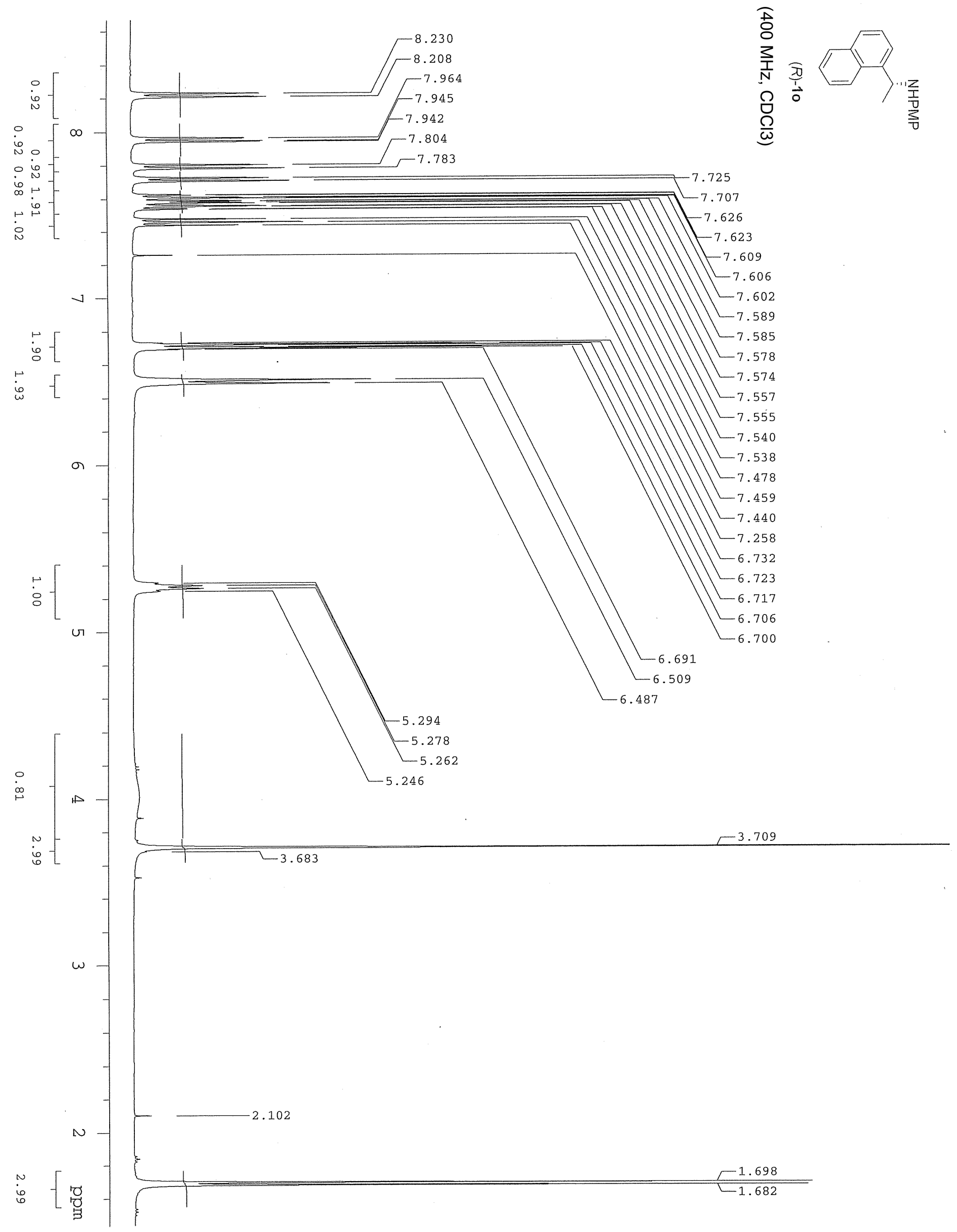




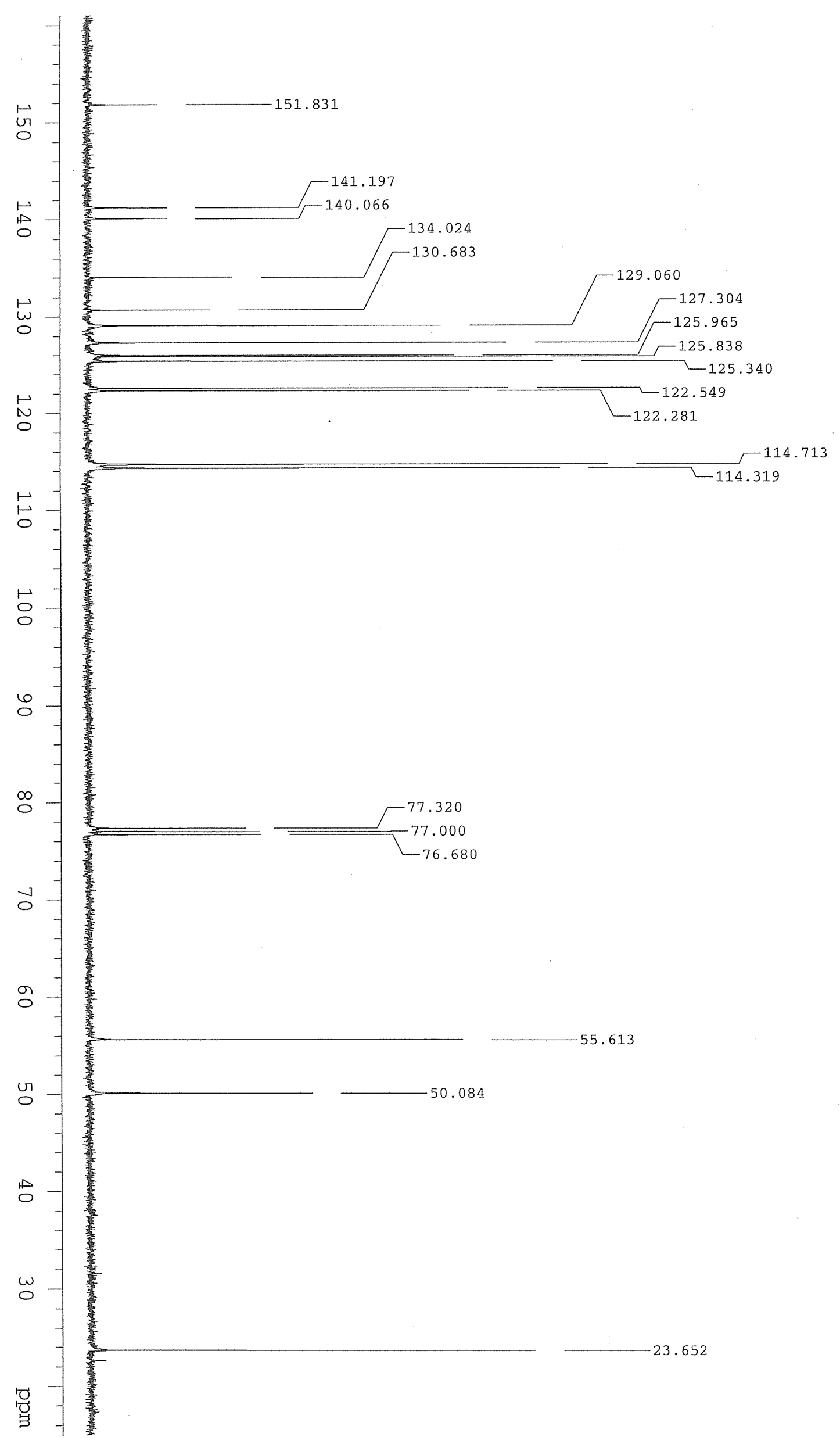

言 
0.05
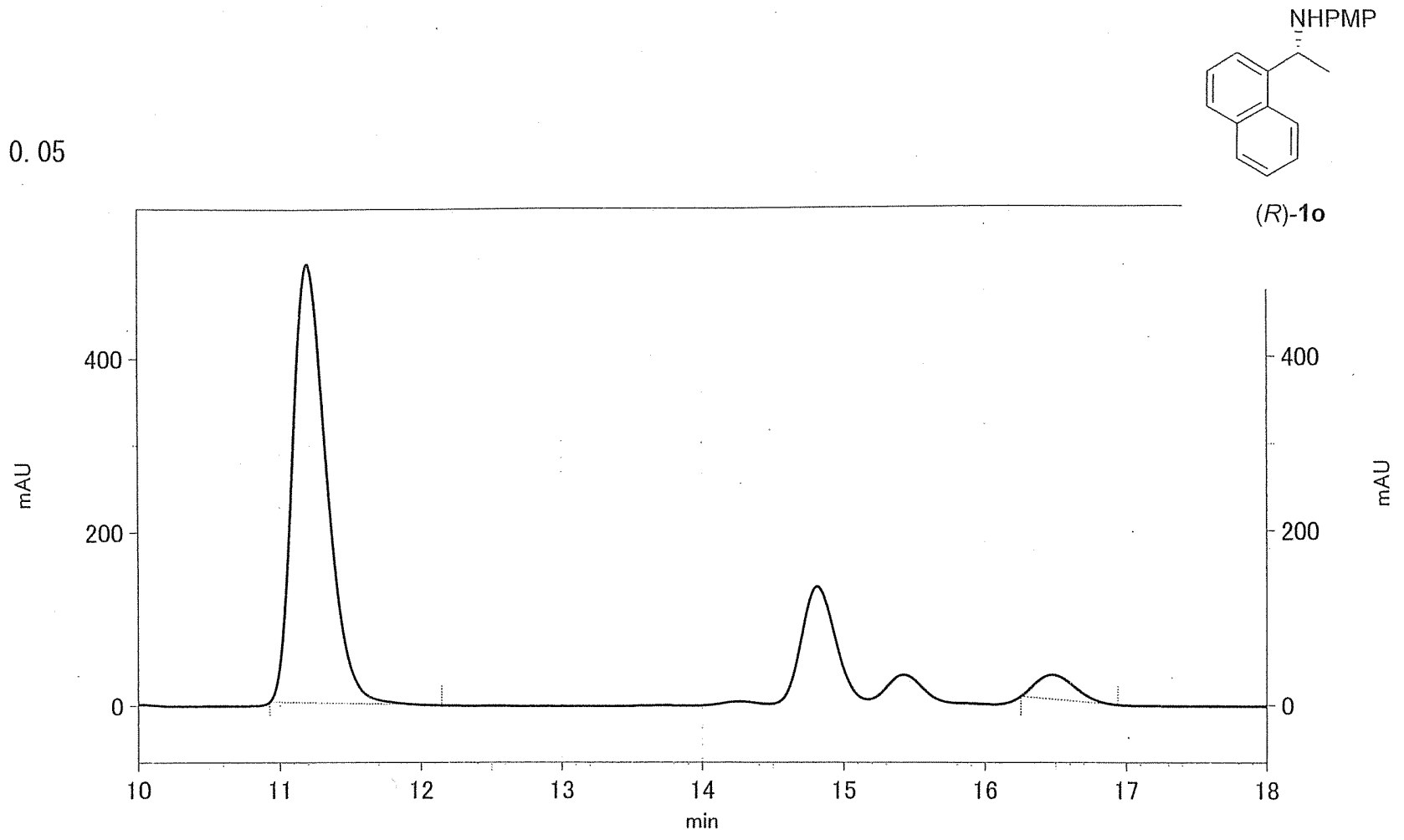

\begin{tabular}{ccccc} 
Peak \# & Retention time & Type & Area & Area \% \\
\hline 1 & 11.20 & $B B$ & 33981638 & 94.054 \\
2 & 16.47 & $B B$ & 2148306 & 5.946
\end{tabular}

0.05
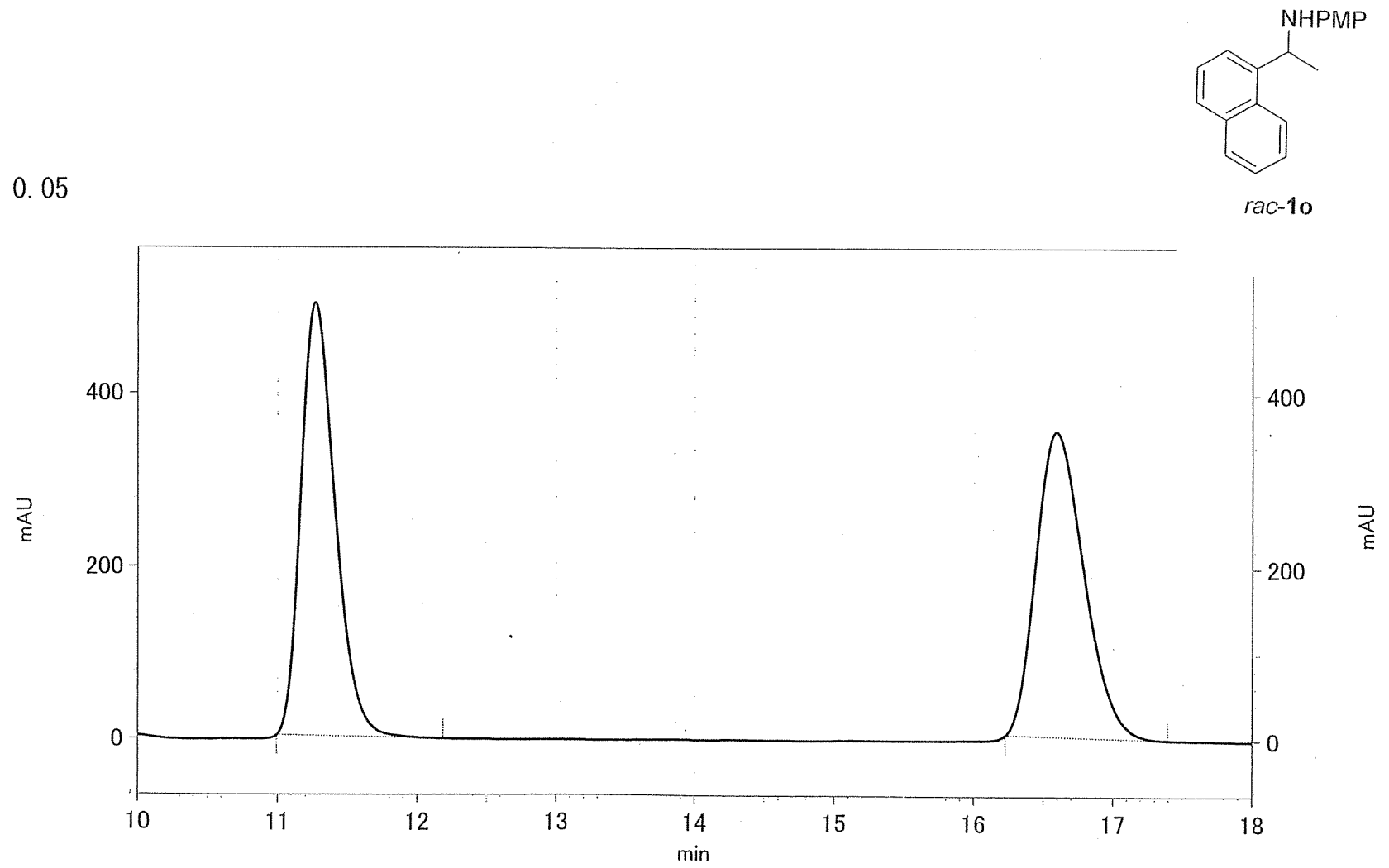

\begin{tabular}{ccccc} 
Peak \# & Retention time & Type & Area & Area \% \\
\hline 1 & 11.27 & BB & 34348917 & 50.267 \\
2 & 16.59 & BB & 33984535 & 49.733
\end{tabular}




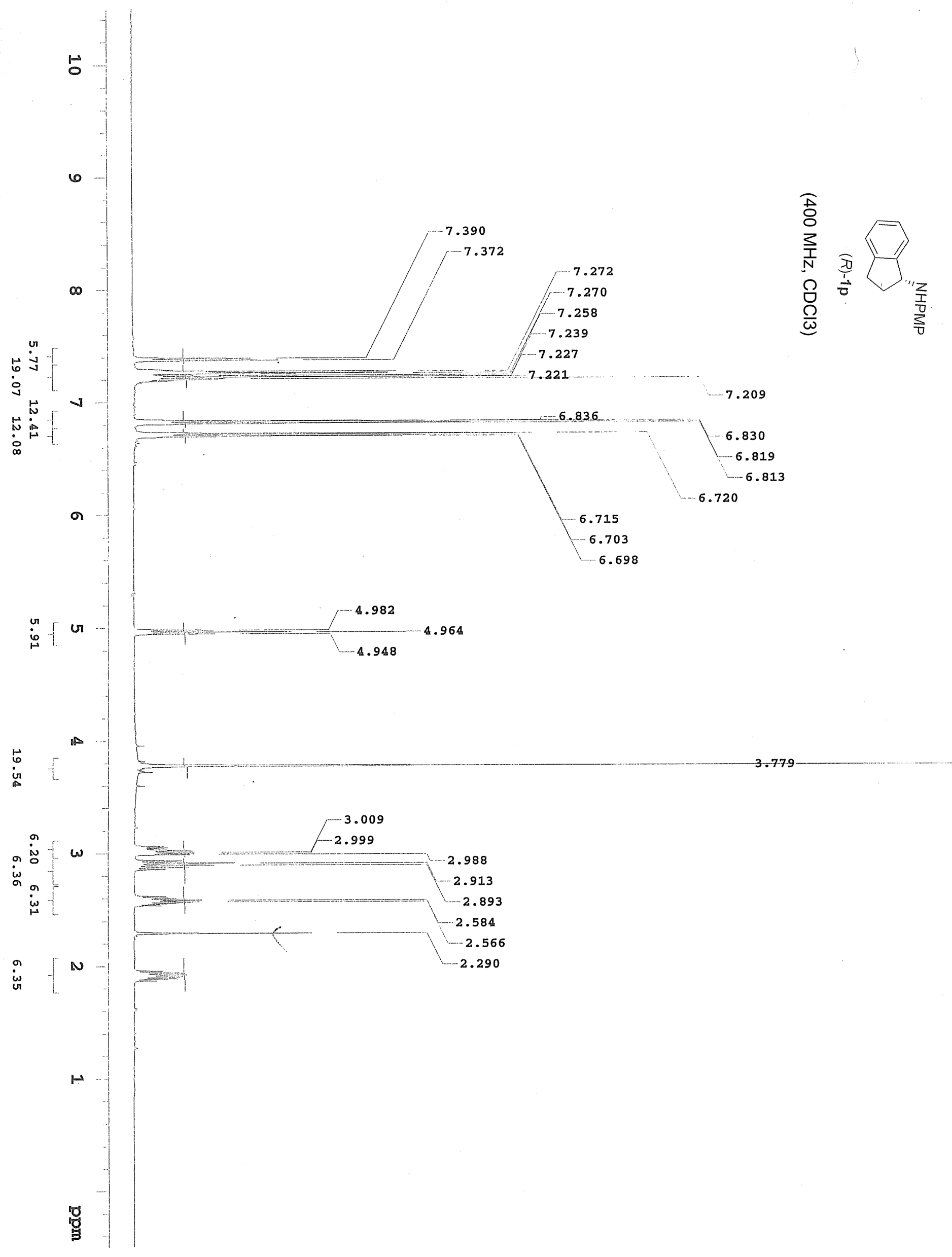




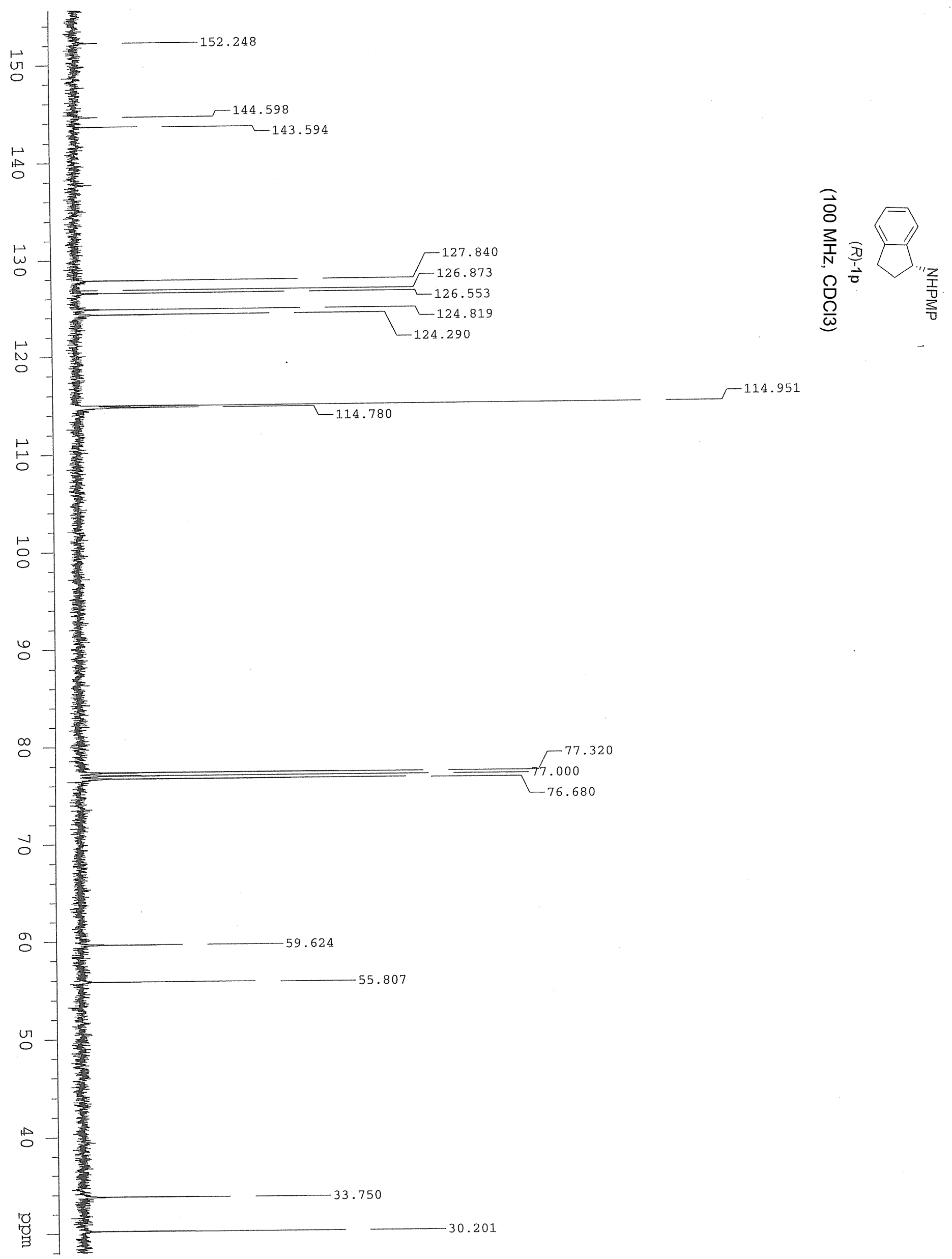


0.05
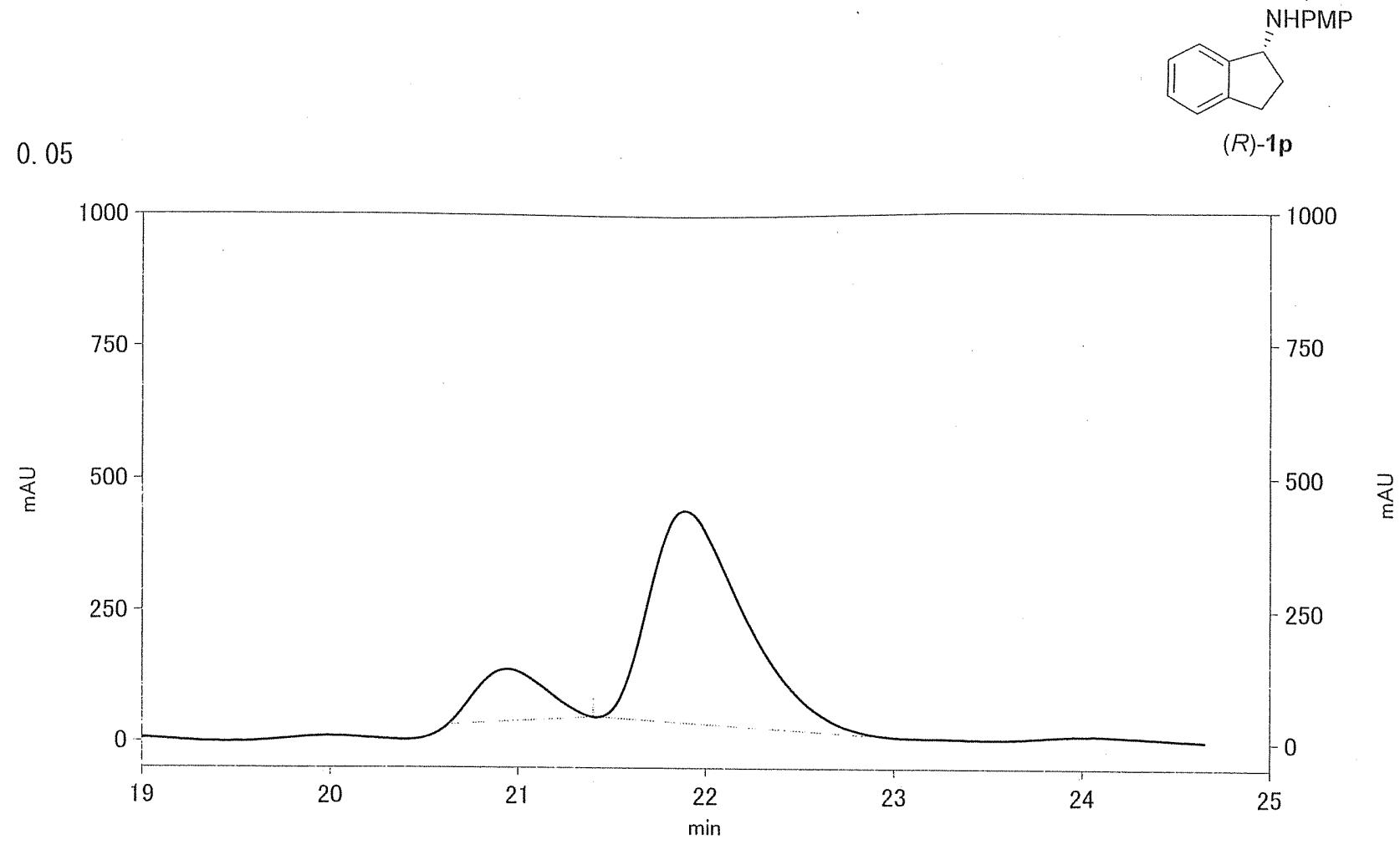

\begin{tabular}{ccccc} 
Peak \# & Retention time & Type & Area & Area \% \\
\hline 1 & 20.94 & II & 10070365 & 15.032 \\
2 & 21.89 & IB & 56923455 & 84.968
\end{tabular}

0.05
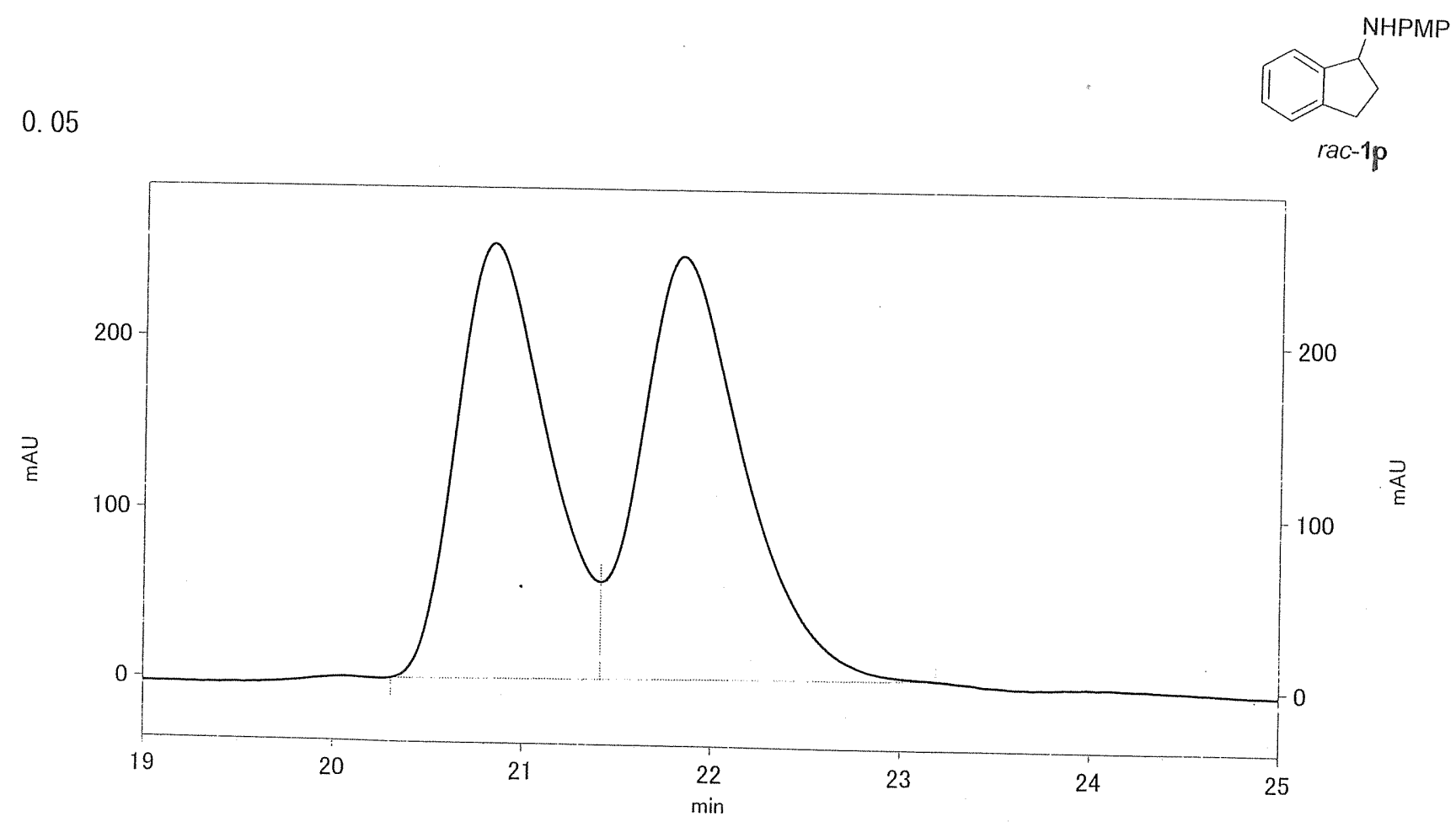

\begin{tabular}{ccccc} 
Peak \# & Retention time & Type & Area & Area \% \\
\hline 1 & 20.83 & BV & 34017861 & 47.487 \\
2 & 21.83 & VB & 37618862 & 52.513
\end{tabular}




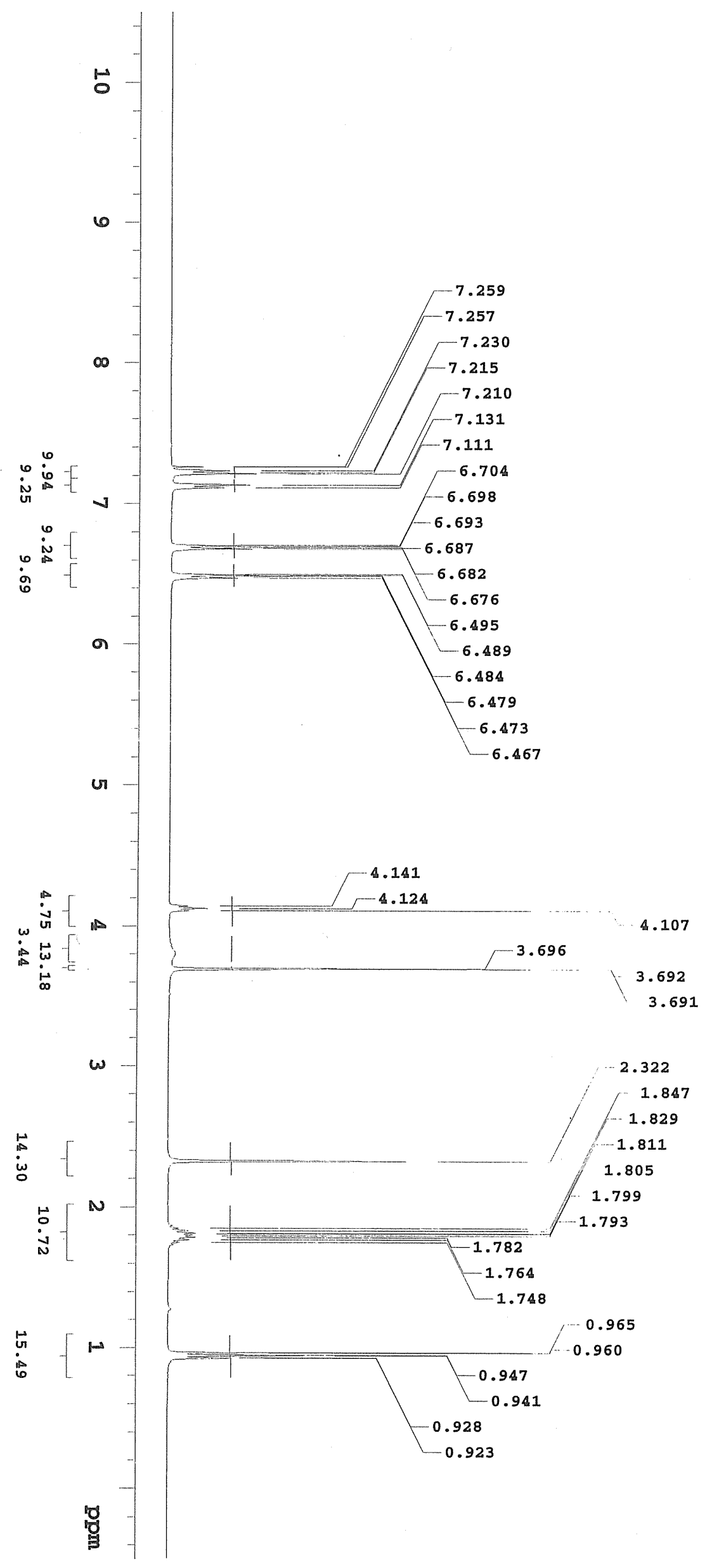




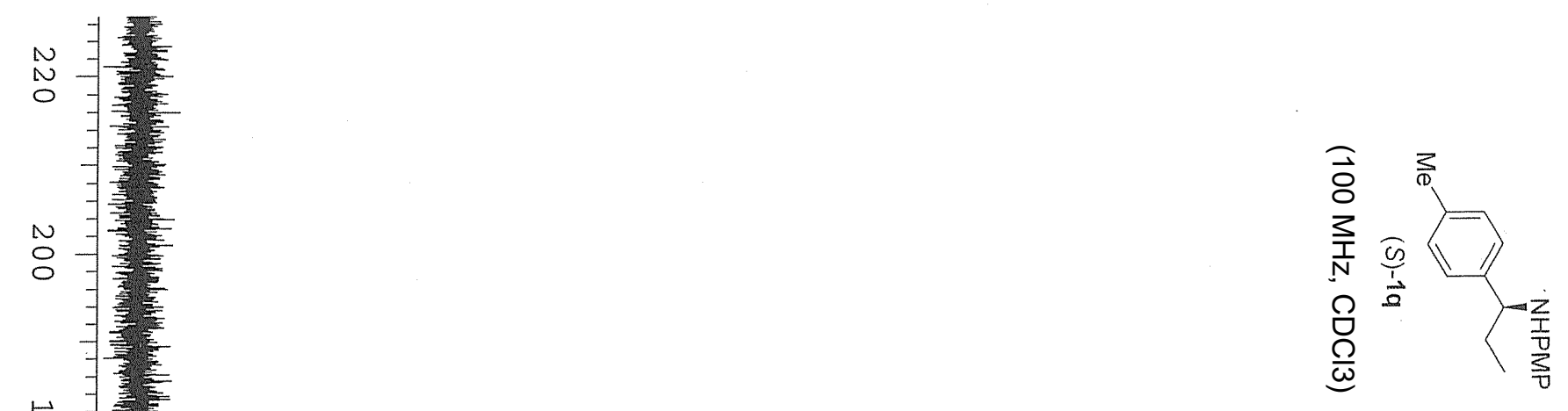

몽

占

点.

$-136.286$

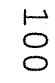

$\stackrel{\infty}{\circ}$

=

=

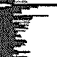

-

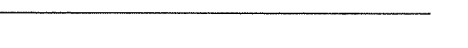

$\longrightarrow 126.404$

$-114.721$

$-114.393$

응

氺

60.227

$-55.725$

\&

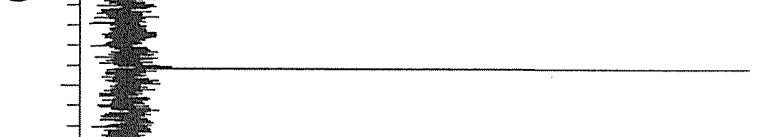

웅

21.048

$-31.667$

'ర్

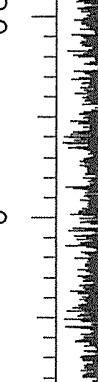


0.05<smiles>CC[C@H](N=PN)c1ccc(C)cc1</smiles>

(S)-1q

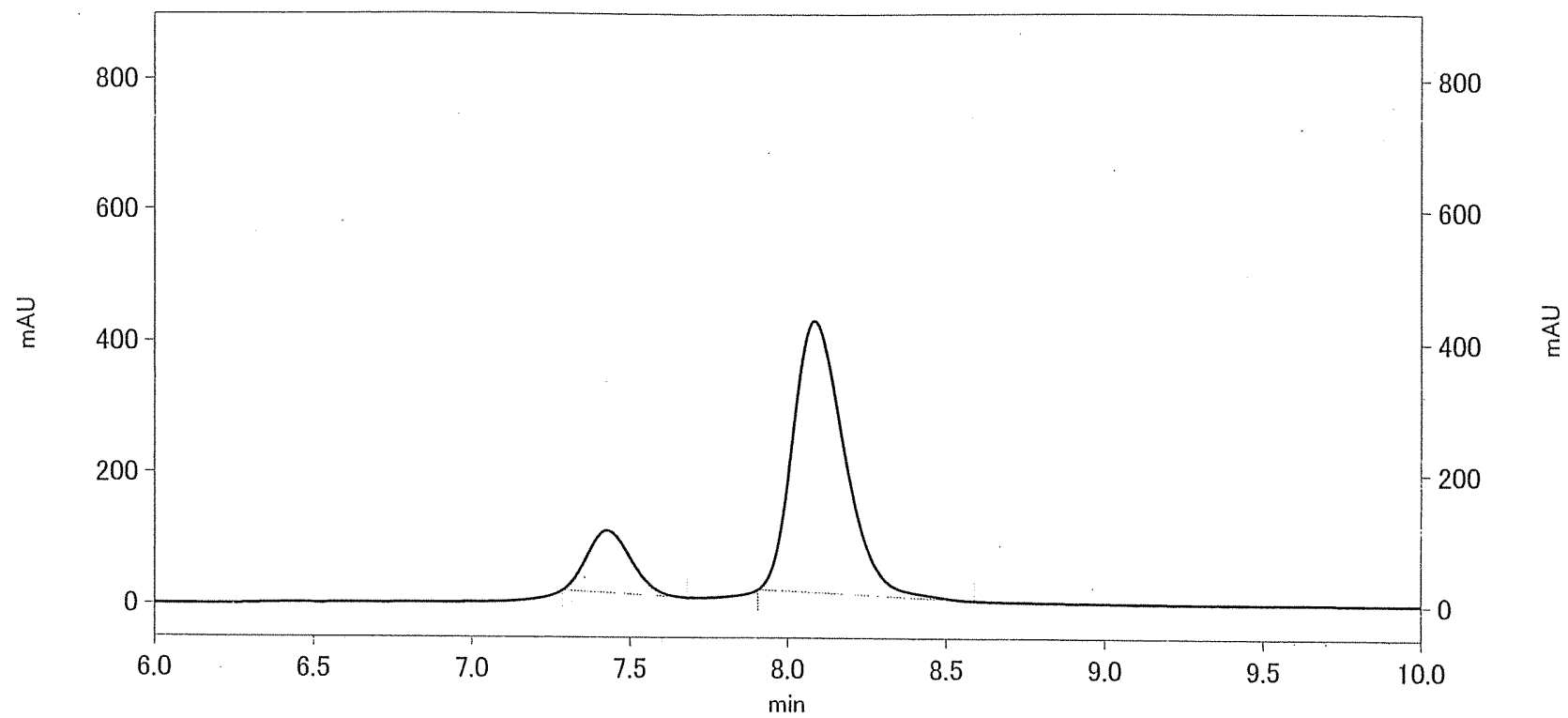

\begin{tabular}{ccccc} 
Peak \# & Retention time & Type & Area & Area \% \\
\hline 1 & 7.43 & BI & 3687326 & 16.429 \\
2 & 8.09 & BB & 18756090 & 83.571
\end{tabular}

0.05
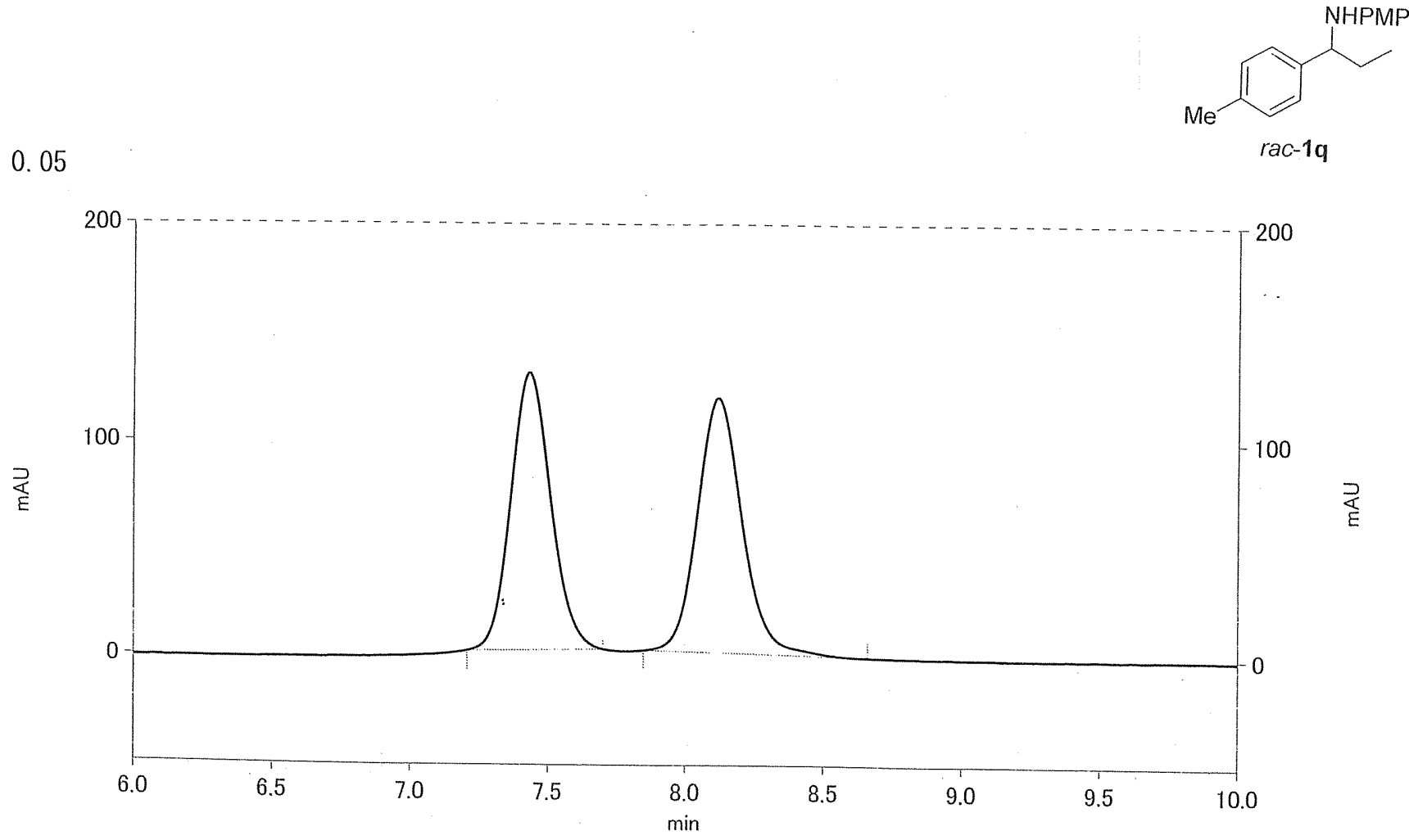

Peak \# Retention time

7. 43

Type 


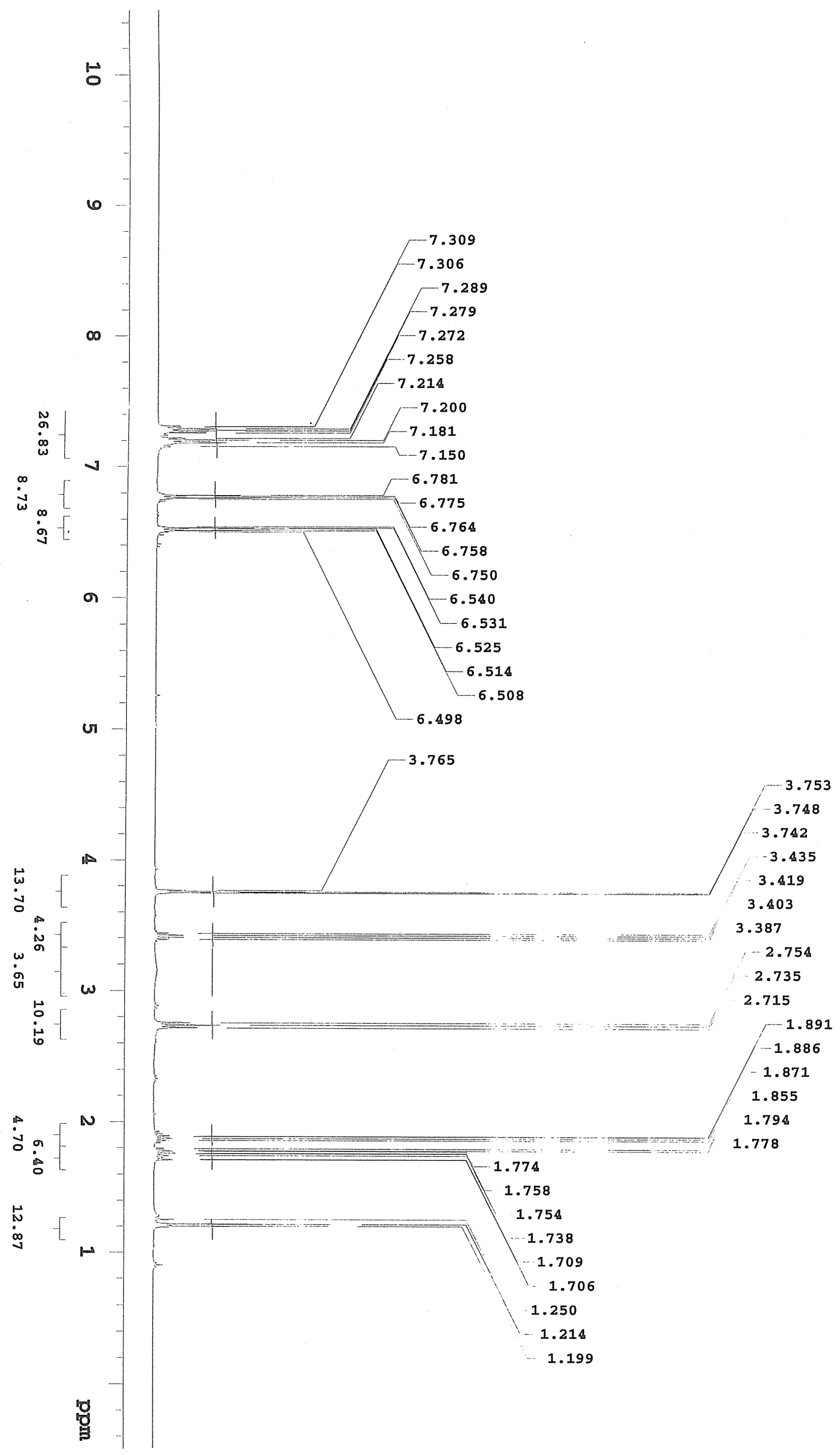




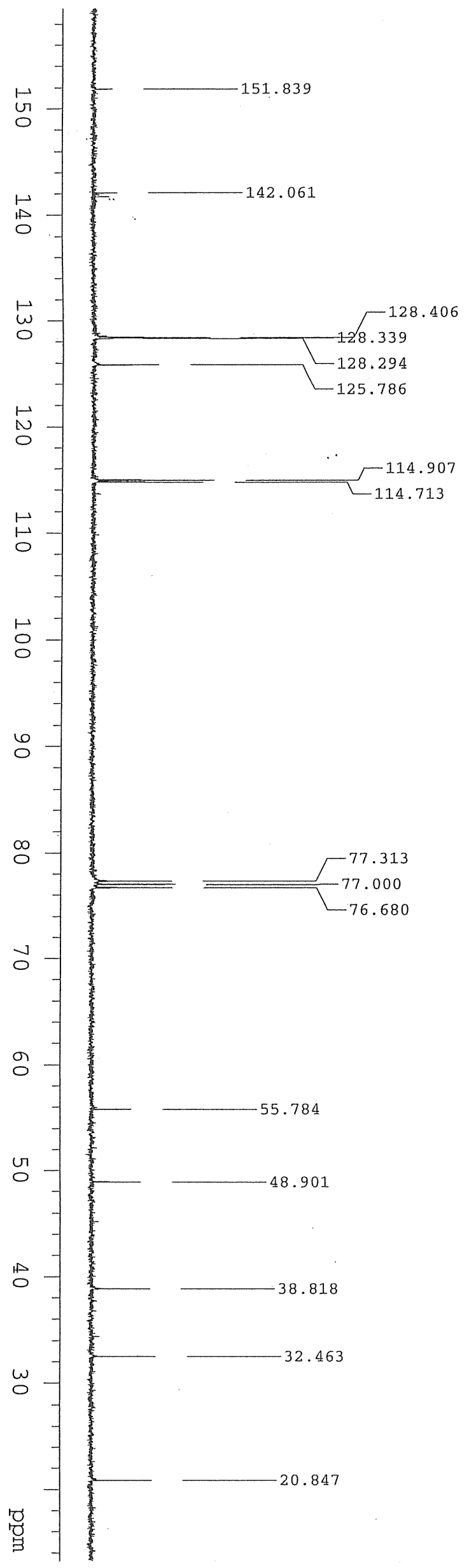


0.05

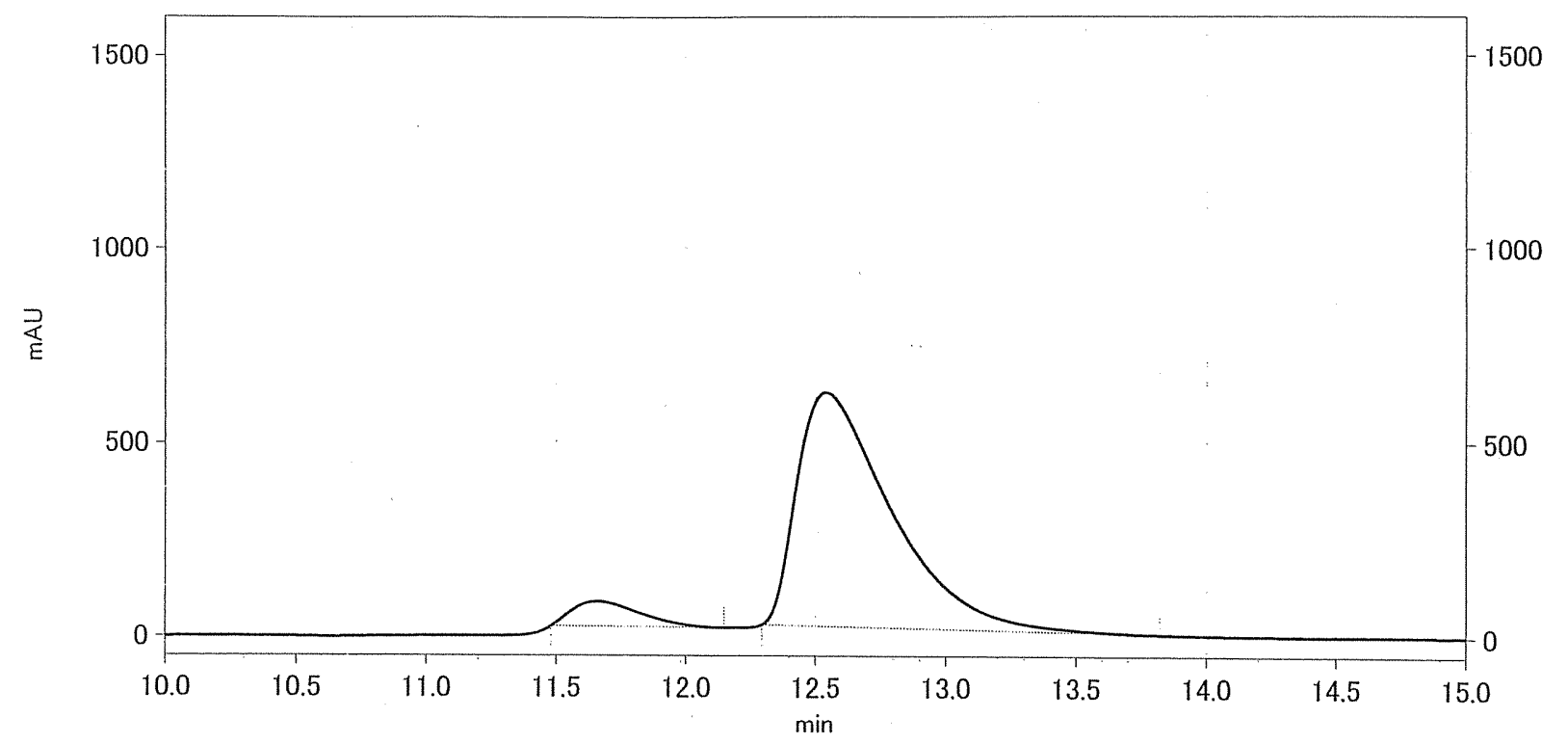

\begin{tabular}{ccccc} 
Peak \# & Retention time & Type & Area & Area \% \\
\hline 1 & 11.66 & IB & 4711237 & 7.184 \\
2 & 12.54 & BB & 60867204 & 92.816
\end{tabular}

0.05
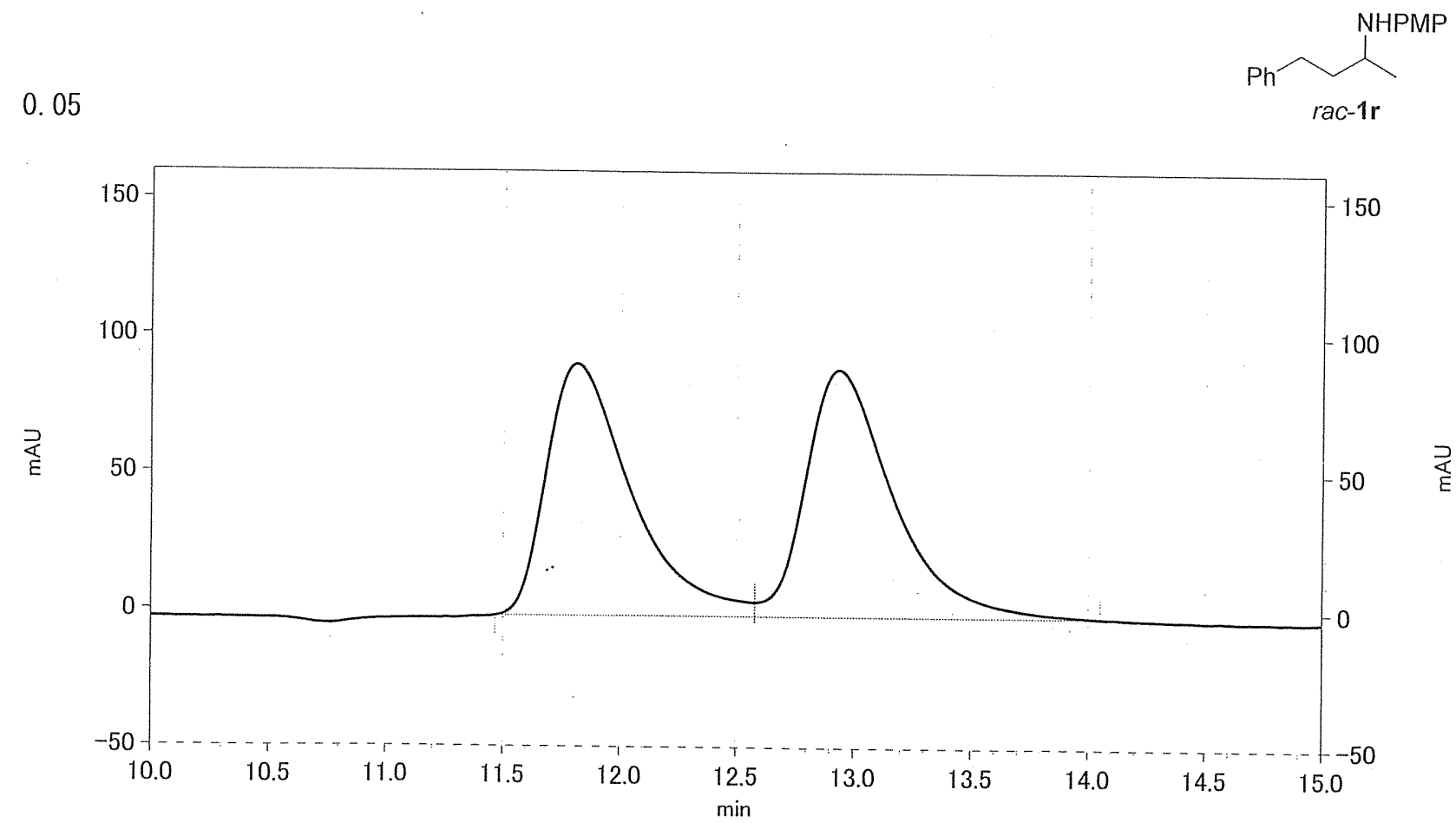

Peak \# Retention time

111.81

2

12. 93

Type

VB
Area

9316612

9391648
Area \%

49.799

50. 201 


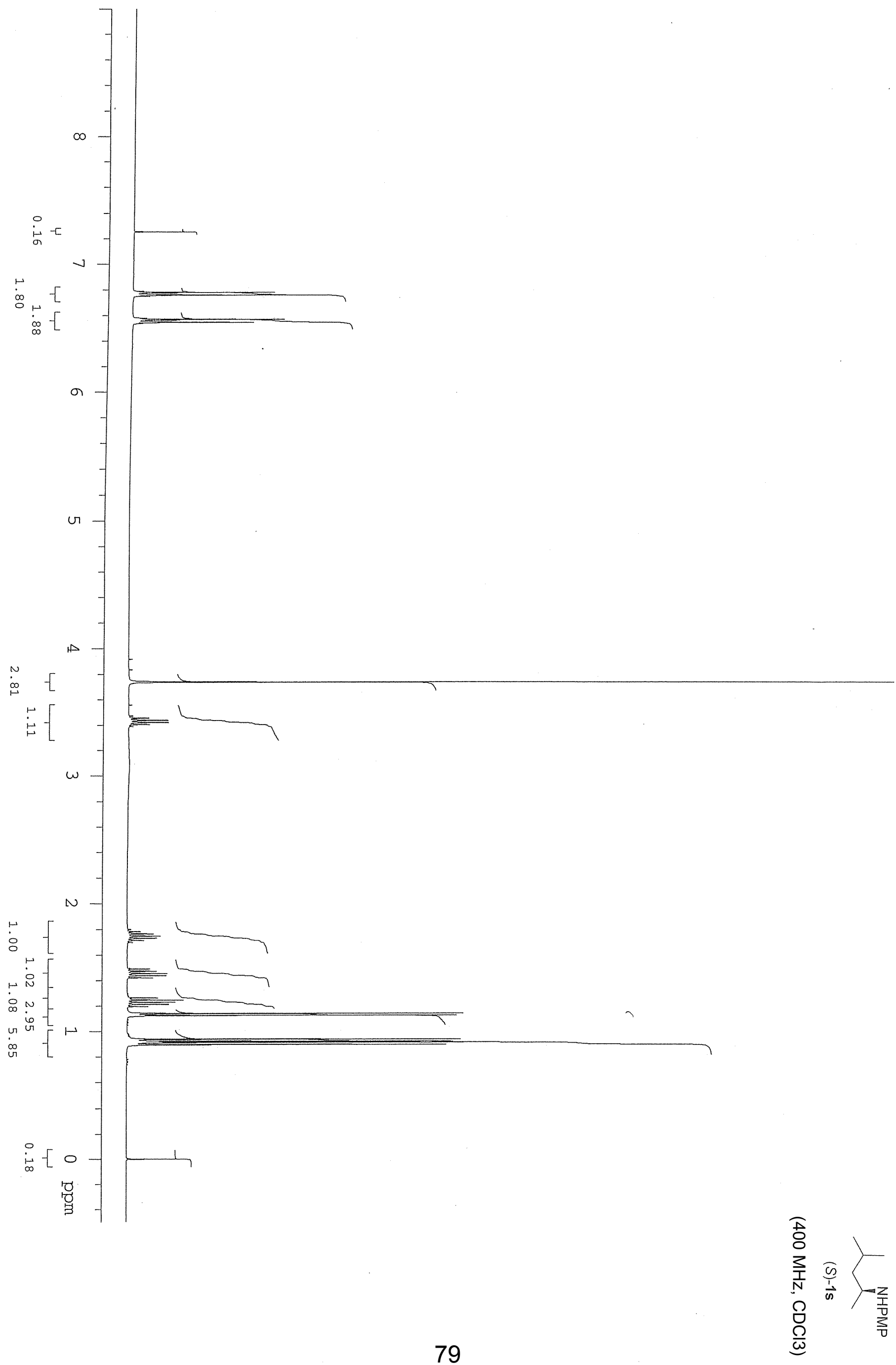




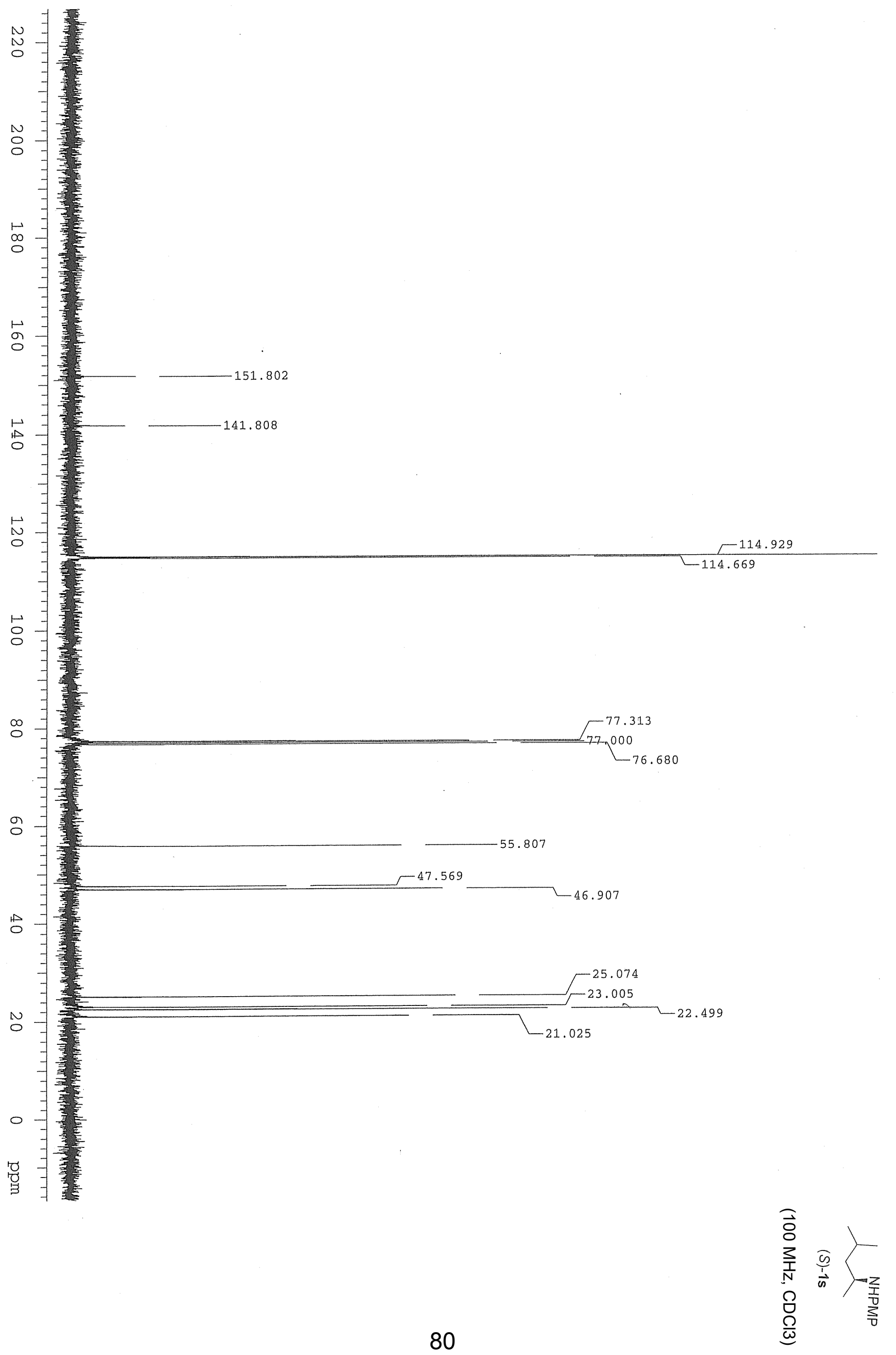




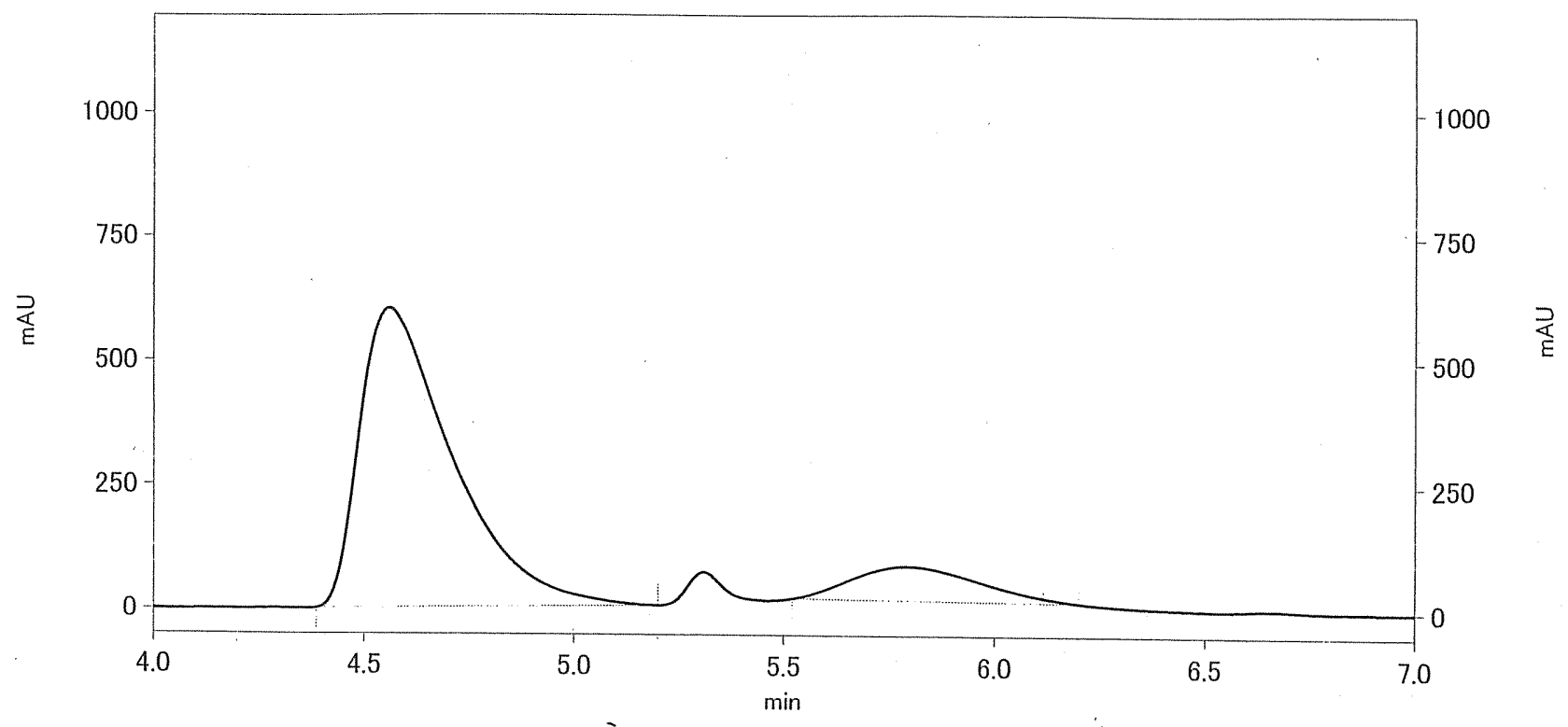

\begin{tabular}{ccccc} 
Peak \# & Retention time & Type & Area & Area \% \\
\hline 1 & 4.56 & $\mathrm{BI}$ & 36948743 & 86.360 \\
2 & 5.79 & $\mathrm{BI}$ & 5835723 & 13.640
\end{tabular}

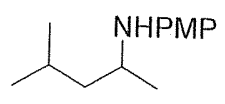

rac-1s

0.05

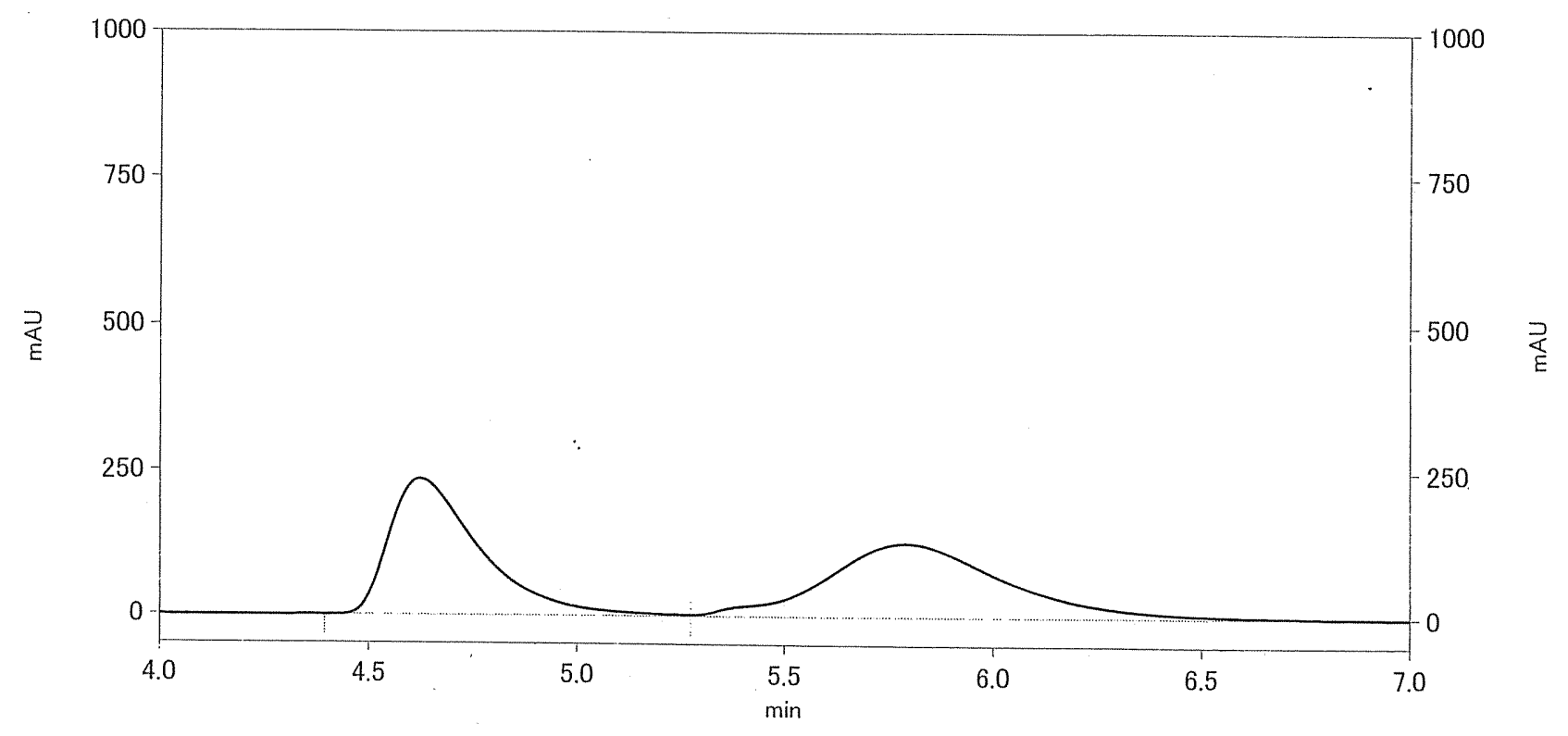

Peak \#

Retention time

$\begin{array}{ll}1 & 4.62 \\ 2 & 5.79\end{array}$

Type

Area

14411912

15524023

Area \%

VI

48.143

51.857

81 


\section{References}

(1) Bowman, R. K.; Johnson J. S. Lewis Acid Catalyzed Dipolar Cycloadditions of an Activated Imidate. J. Org. Chem. 2004, 69, 8537-8540.

(2) Saito, K.; Shibata, Y.; Yamanaka, M.; Akiyama, T.Chiral Phosphoric Acid-Catalyzed Oxidative Kinetic Resolution of Indolines Based on Transfer Hydrogenation to Imines J. Am. Chem. Soc. 2013, 135, 11740-11743.

(3) Zhu, X.; Du, H. A chiral borane catalyzed asymmetric hydrosilylation of imines. Org. Biomol. Chem. 2015, 13, 1013-1016.

(4) Malkov, A. V.; Vranková, K.; Stončius, S.; Kočovský P. Asymmetric Reduction of Imines with Trichlorosilane, Catalyzed by Sigamide, an Amino Acid-Derived Formamide: Scope and Limitations. J. Org. Chem. 2009, 74, 5839-5849.

(5) Storer, R. I.; Carrera, D. E.; Ni, Y.; MacMillan, D. W. C. Enantioselective Organocatalytic Reductive Amination. J. Am. Chem. Soc. 2006, 128, 84-86.

(6) Chi, Y.; Zhou, Y.-G.; Zhang, X. Highly Enantioselective Reductive Amination of Simple Aryl Ketones Catalyzed by Ir-f-Binaphane in the Presence of Titanium(IV) Isopropoxide and Iodine. J. Org. Chem. 2003, 68, 4120-4122.

(7) Denmark, S. E.; Nakajima, N.; Nicaise, O. J.-C. Asymmetric Addition of Organolithium Reagents to Imines. J. Am. Chem. Soc. 1994, 116, 8797-8798.

(8) Saito, K.; Horiguchi, K.; Shibata, Y.; Yamanaka, M.; Akiyama, T.

Chiral Phosphoric - Acid - Catalyzed Transfer Hydrogenation of Ethyl Ketimine Derivatives by Using Benzothiazoline. Chem. Eur. J. 2014, 20, 7616-7620.

(9) Saito, K.; Akiyama, T. Enantioselective organocatalytic reductive amination of aliphatic ketones by benzothiazoline as hydrogen donor. Chem. Commun. 2012, 48, 4573-4575.

(10) Gaussian 16, Revision B.01, Frisch, M. J.; Trucks, G. W.; Schlegel, H. B.; Scuseria, G. E.; Robb, M. A.; Cheeseman, J. R.; Scalmani, G.; Barone, V.; Petersson, G. A.; Nakatsuji, H.; Li, X.; Caricato, M.; Marenich, A. V.; Bloino, J.; Janesko, B. G.; Gomperts, R.; Mennucci, B.; Hratchian, H. P.; Ortiz, J. V.; Izmaylov, A. F.; Sonnenberg, J. L.; Williams-Young, D.; Ding, F.; Lipparini, F.; Egidi, F.; Goings, J.; Peng, B.; Petrone, A.; Henderson, T.; Ranasinghe, D.; Zakrzewski, V. G.; Gao, J.; Rega, N.; Zheng, G.; Liang, W.; Hada, M.; Ehara, M.; Toyota, K.; Fukuda, R.; Hasegawa, J.; Ishida, M.; Nakajima, T.; Honda, Y.; Kitao, O.; Nakai, H.; Vreven, T.; Throssell, K.; Montgomery, Jr. J. A.; Peralta, J. E.; Ogliaro, F.; Bearpark, M. J.; Heyd, J. J.; Brothers, E. N.; Kudin, K. N.; Staroverov, V. N.; Keith, T. A.; Kobayashi, R.; Normand, J.; Raghavachari, K.; Rendell, A. P.; Burant, J. C.; Iyengar, S. S.; 
Tomasi, J.; Cossi, M.; Millam, J. M.; Klene, M.; Adamo, C.; Cammi, R.; Ochterski, J. W.; Martin, R. L.; Morokuma, K.; Farkas, O.; Foresman, J. B.; Fox, D. J.; Pople, J. A. Gaussian, Inc.: Wallingford CT, 2016.

(11) Shibata, Y.; Yamanaka, M. DFT Study of Mechanism and Origin of Enantioselectivity in Chiral BINOL-Phosphoric Acid Catalyzed Transfer Hydrogenation of Ketimine and $\alpha$-Imino Ester Using Benzothiazoline J. Org. Chem. 2013, 78, 3731-3736. 